\title{
On the Search for the Neural Correlates of Unified Consciousness
}

\author{
Alain El Hofi, B.A. Honours
}

A thesis submitted to the Faculty of Graduate Studies and Research in partial fulfillment of the requirements for the degree of

\author{
Master of Arts \\ Department of Philosophy
}

\author{
Carleton University \\ Ottawa, Ontario \\ May $14^{\text {th }}, 2008$ \\ (C) 2008, Alain El Hofi
}




$\begin{array}{ll}\begin{array}{l}\text { Library and } \\ \text { Archives Canada }\end{array} & \begin{array}{l}\text { Bibliothèque et } \\ \text { Archives Canada }\end{array} \\ \begin{array}{l}\text { Published Heritage } \\ \text { Branch }\end{array} & \begin{array}{l}\text { Direction du } \\ \text { Patrimoine de l'édition }\end{array} \\ \begin{array}{l}\text { 395 Wellington Street } \\ \text { Ottawa ON K1A 0N4 } \\ \text { Canada }\end{array} & \begin{array}{l}\text { 395, rue Wellington } \\ \text { Ottawa ON K1A 0N4 } \\ \text { Canada }\end{array}\end{array}$

Your file Votre référence ISBN: 978-0-494-40599-4

Our file Notre référence

ISBN: 978-0-494-40599-4

NOTICE:

The author has granted a nonexclusive license allowing Library and Archives Canada to reproduce, publish, archive, preserve, conserve, communicate to the public by telecommunication or on the Internet, loan, distribute and sell theses worldwide, for commercial or noncommercial purposes, in microform, paper, electronic and/or any other formats.

The author retains copyright ownership and moral rights in this thesis. Neither the thesis nor substantial extracts from it may be printed or otherwise reproduced without the author's permission.
AVIS:

L'auteur a accordé une licence non exclusive permettant à la Bibliothèque et Archives Canada de reproduire, publier, archiver, sauvegarder, conserver, transmettre au public par télécommunication ou par l'Internet, prêter, distribuer et vendre des thèses partout dans le monde, à des fins commerciales ou autres, sur support microforme, papier, électronique et/ou autres formats.

L'auteur conserve la propriété du droit d'auteur et des droits moraux qui protège cette thèse. $\mathrm{Ni}$ la thèse ni des extraits substantiels de celle-ci ne doivent être imprimés ou autrement reproduits sans son autorisation.
In compliance with the Canadian Privacy Act some supporting forms may have been removed from this thesis.

While these forms may be included in the document page count, their removal does not represent any loss of content from the thesis.
Conformément à la loi canadienne sur la protection de la vie privée, quelques formulaires secondaires ont été enlevés de cette thèse.

Bien que ces formulaires aient inclus dans la pagination, il n'y aura aucun contenu manquant.

\section{Canada}




\begin{abstract}
Our project is about the methodological and conceptual problems raised by the search for the neural correlates of consciousness (NCCs), and in particular those posed by the neural correlates of unified forms of consciousness. Using the framework of mechanistic explanation, we examine/evaluate different approaches to NCCs as to how they fare at bringing us closer to the neural mechanisms underlying the unity of consciousness. Based on our analysis of the work done by researchers attempting to discover the NCC for the function of "individual object unity," we argue that they fail to distinguish this function from another related, but different, function of unified consciousness (the "multiple object unity" function). Building on recent experimental work done in another context, we show how these two functions can be isolated, and we suggest further experimental interventions aimed at pinning down the structural and functional properties of the mechanisms underlying these functions.
\end{abstract}




\section{Acknowledgments}

I am very grateful to my parents for all their support over the years. To Janine Laurencin, for her unwavering help and encouragement: merci infiniment! I am also very grateful to Raouf El Hofi for his encouragement and for ensuring that I may have opportunities which were not available to him.

I am extremely grateful to Andy Brook for countless stimulating conversations and always making time to discuss ideas or read drafts. His guidance and encouragement have been invaluable.

I would like to thank Jay Drydyk, Dave Matheson, and John Logan for agreeing to be on my committee and for their interesting comments and questions. I would also like to thank Sandy Kirkpatrick for her kind help.

Thanks to my friends: André, Anne, Mark, Martine, Mélissa, Jen, Sam, and Yvette.

Most of all I would like to thank ma belle Julie for all her loving support and for making me feel like the luckiest person alive. 


\section{Table of Contents}

Title Page

Abstract

Acknowledgments

iii

Table of Contents

iv

List of Tables

vi

List of Figures

\section{Chapter 1}

1.1 Introduction

1.2 The Anti-Functionalist Challenge:

Chalmers' Zombie Thought Experiment 4

1.3 Recent Work on the NCCs 16

1.4 Our Contribution 20

1.5 Short Summary of Upcoming Chapters 20

\section{Chapter 2}

2.1 Introduction 22

$2.2 \quad$ General Types of Consciousness 22

2.3 Will What We Learn from Visual Consciousness

Generalize to Other Modalities? 24

2.4 What is a Neural Correlate of Consciousness? 25

2.5 "Content" NCCs 27

2.6 How to Find the NCCs:

general strategies, methodological problems

(lesion studies, ascriptions of consciousness) 33

2.7 The Role of NCCs in Consciousness Studies 44

2.8 Mechanistic Explanation: Methodological Issues Decomposing Mechanisms and

Levels of Analysis

\section{Chapter 3}

3.1 Introduction

3.2 The Notion of Unity: Two Forms of Unity and Gestalt Relationships

3.3 Phenomenal Unity Implies the Integration of Distributed Information on the

Neurological Level

3.4 Methodological Problem: What is the

Right Level of Analysis? 
3.5 One Source of the Correct Level of Analysis

Problem: Neural Coding

65

3.6 How Neurons Could Encode Information 66

3.7 The "Explanatory Gap" (or the "Hard Problem") 73

3.8 Local Approaches, Firing Rate Coding, and Unity 74

3.9 Could Receptive Field Analyses Lead the Way to

3.10 Conclusion

a Mechanistic Explanation of Unity? $\quad 77$

\section{Chapter 4}

$\begin{array}{lll}4.1 & \text { Introduction } & 86\end{array}$

4.2 Synchrony in General 86

4.3 How Synchrony is Measured 88

4.4 Spontaneous Oscillations vs. Oscillations

Elicited by Specific Stimuli $\quad 89$

4.5 Synchrony: A Necessary and/or Sufficient
Condition for Consciousness?

4.6 Synchrony as a Putative Mechanism of Unity 94

4.7 What Can Synchrony Tell Us About the

Mechanisms of Unified Consciousness? 100

4.8 The Imaging Techniques of fMRI and PET are

Better for Discovering a Mechanism's

Structural and Functional Properties

103

4.9 A More Fine-Grained Paradigm (Isolating the

"Individual Object Unity" Function)

4.10 A More Fine-Grained Paradigm - Second Set of Experiments (Isolating the NCC of the "Individual Object Unity" Function) 110

4.11 Transcranial Magnetic Stimulation (TMS) 111

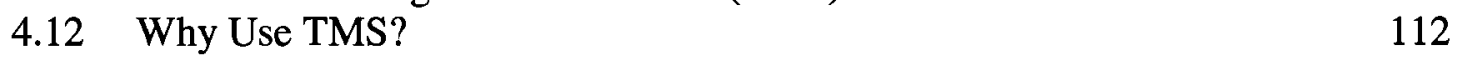

$\begin{array}{ll}4.13 \text { Conclusion } & 115\end{array}$

$\begin{array}{ll}\text { Notes } & 117\end{array}$

$\begin{array}{ll}\text { References } & 122\end{array}$

List of Sources of Tables and Figures 133

Glossary of Science-Related Terms 134 


\section{List of Tables}

Table 1 - Binary Numbers $\quad 69$

$\begin{array}{ll}\text { Table 2 - Binary Addition } & 69\end{array}$

Table 3 - Binary Addition $\quad 69$

(Input-Output Possibilities)

Table 4 - "And" Circuit $\quad 70$

(Input-Output Possibilities)

Tables 5-7 - "Exclusive-Or" Circuit

(Input-Output Possibilities) 


\section{List of Figures}

$\begin{array}{ll}\text { Figure } 1 \text { - Pac-Man } & 60\end{array}$

$\begin{array}{ll}\text { Figure 2 - Kanisza Triangle } & 60\end{array}$

$\begin{array}{ll}\text { Figure 3 - Neuron } & 67\end{array}$

$\begin{array}{ll}\text { Figure 4 - "And" Circuit } & 70\end{array}$

Figure 5 - "Exclusive-Or" Circuit $\quad 70$

Figure 6 - Two-Bit Neuronal Adder Circuit $\quad 72$

Figure 7 - The Receptive Field of an "On-Center" Cell 80

Figure 8 - Simple Cell Circuit $\quad 82$

Figure 9-Complex Cell Circuit $\quad 82$

Figure 10 - Stimuli Used in Illusory Masked Priming Paradigm 106 


\section{On the Search for the Neural Correlates of Unified Consciousness}

\section{Chapter 1}

\section{1 - Introduction}

Our project is about the methodological and conceptual problems raised by the search for the neural correlates of consciousness (NCCs), and in particular those posed by the neural correlates of unified forms of consciousness. In daily life, consciousness is what matters most to us about the mental. The qualitative aspects of consciousness (i.e., "what it is like" to feel pain or to have the experience of seeing blue) are important challenges to a physical or neurological explanation of consciousness. Many agree that identifying the NCCs is the first step in a scientific approach to consciousness (Hohwy, 2007, p. 461; Crick \& Koch, 1998, p. 97; Heywood \& Milner, 2002, p. 241). The main drive behind NCC research is the hope that their discovery will lead to a better understanding of the relations between neural states and conscious states. This question of the relations between neural states and conscious states can be considered as a contemporary reformulation of the "mind-body problem" made famous by Descartes.

Recently, there has been work on neural correlates of consciousness with an explicit focus on unified consciousness (Rodriguez et al., 1999; Tallon-Baudry, 2003a; Engel \& Singer, 2001; Revonsuo et al., 1997; Keil et al., 1999). Unity is a central characteristic of common conscious experience. When one hears a plane passing overhead and feels thirsty, the sounds and the feeling of thirst are not experienced as two separate "smaller" experiences. Rather, both the sound and thirst are experienced as two items which are part of the same unified experience. The unity characteristic of 
consciousness poses special challenges for those trying to understand the relations between brain activity and conscious activity. For example, it is widely believed by neuroscientists that an object's various features (such as its colour, its shape, and its movement) are processed by different areas of the brain (Humphreys, 2003, p. 115). Yet somehow when I look at the coffee mug before me, its various features are experienced as unified. My experience is of a unified object, not of a confused jumble of unrelated features. A complete explanation of consciousness must address problems related to its unified character such as how features are bound into unified objects.

NCC researchers typically try to correlate specific cognitive or conscious functions (such as short-term memory or visual consciousness of colour) with the activity of a particular brain region. The hope is that finding a systematic correlation between a particular brain area's activity and a particular conscious function could be a step towards the discovery of the neural mechanism(s) responsible for this function. In this way, NCCs could play an important role in a functionalist approach to consciousness. We adopt a functionalist materialist position towards the study of consciousness. In a moment we defend the viability of such a position by attempting to refute the main philosophical argument against functionalism about consciousness. But first, let's situate our approach vis-à-vis the other main positions in consciousness studies.

Physicalism is the idea that everything is physical and every event can be explained in terms of physical properties. Physicalists hold that this is true of the mind and consciousness, too. On this account, there is only one type of substance and one type of property. There are other positions that oppose physicalism and yet are broadly 
materialist. Functionalism, for example, considers that the mind is physical, an aspect of the brain, yet holds that mental properties cannot be reduced to physical ones. Functionalists are substance monists like physicalists, but property dualists. The properties that make a state mental are not and cannot be reduced to the properties that make it material.

Functionalism is the leading alternative to physicalism. It holds that mental states are individuated in terms of their causes and effects (their relations with the agents' sensory stimuli, behaviour, and other mental states) (Churchland, 1997, p. 36). Nearly all functionalists are anti-reductive about the mental, insisting that descriptions of mental properties cannot be reduced to descriptions of physical properties ("anomalous monism"). Even though it considers all mental events to be material events, anomalous monism claims that reduction fails because concepts of mental kinds ("belief," for example) cannot be mapped onto concepts for material kinds ("brain state of type ABC," for example) (Davidson, 1970, p. 214). Some philosophers who are functionalists about the mental in general go farther with consciousness and make the anti-functionalist claim that consciousness cannot be reduced to function (Chalmers, 1996, p. 97). Because research on the NCCs proceeds by correlating neural activity with consciousness understood in terms of functions, this claim must be taken seriously. Thus, before we can properly begin our investigation of the NCCs, we should deal with critics of functionalist theories of consciousness. Using Chalmers as its representative, we answer to the antifunctionalist challenge by attempting to refute zombie thought experiments. 


\section{2 - The anti-functionalist challenge: Chalmers' zombie thought experiment}

Chalmers uses a zombie thought experiment in an attempt to show that consciousness resists functional explanation, and concludes from this that functionalism about consciousness is false. In the literature, "consciousness" is used to denote a wide range of phenomena. In this chapter "consciousness," refers to self-consciousness or consciousness of one's own representations. In particular, zombie thought experiments pertain to "phenomenal consciousness" (which can be thought of as a qualitative aspect of consciousness or "what it is like" to be conscious). After a detailed examination of David Chalmers' zombie thought experiment, we consider two strands of objections that challenge the viability of such thought experiments, as well as a possible reply from zombie defenders.

In The Conscious Mind, David Chalmers argues that facts about consciousness do not logically supervene on physical or functional facts. (That is, all the physical/functional facts do not entail all facts about consciousness, in the sense that it would not be contradictory to imagine a situation where all the physical/functional facts of our world are instantiated, without there also being any conscious properties.) Before examining this claim in more detail and seeing how Chalmers concludes from it that functionalism about consciousness is false, some preliminary terminological elucidation is in order. We start with the notions of supervenience and possibility.

In general, supervenience is a relation of dependence holding between two sets of properties. High-level properties supervene on lower-level properties which are considered to be more fundamental. According to Chalmers' definition: “B-properties 
supervene on A-properties if no two possible situations are identical with respect to their A-properties while differing in their B-properties" (Chalmers, 1996, p. 33). This notion further subdivides into many types, depending on which notion of possibility one associates it to. Three kinds of supervenience will concern us here: logical, natural, and metaphysical. These types of supervenience mirror three types of possibility: logical, natural, and metaphysical possibility.

Logical (or conceptual) supervenience is defined as “B-properties supervene logically on A-properties if no two logically possible situations are identical with respect to their A-properties but distinct with respect to their B-properties" (Chalmers, 1996, p. 35). Here, Chalmers notes that possibility roughly corresponds to the notion of conceivability.

Hume also thought something similar about the relation between conceivability and possibility. In his Treatise he writes, "'Tis an establish'd maxim in metaphysics, that whatever the mind clearly conceives, includes the idea of possible existence, or in other words, that nothing we imagine is absolutely impossible" (Hume, 1978, p. 32) (italics in original). He goes on to say that since we can imagine a "golden mountain" such a mountain is possible. However, since we cannot imagine a "mountain with no valley," a mountain with no valley is impossible. While a world in which, ceteris paribus, rocks "fall" upwards is logically possible, ${ }^{1}$ one where bachelors are married is not. The latter case is logically impossible because the property of bachelorhood supervenes on that of not being married. The former case, however, even if not possible in our world, violates no logical law (it exhibits no apparent contradiction). 
Natural supervenience corresponds to empirical possibility in our actual world. Bproperties supervene naturally on A-properties if any two naturally possible situations (i.e., that could occur without violating natural laws) which have the same A-properties, also have the same B-properties (Chalmers, 1996, p. 36). While rocks that rise up when we let go of them are, as we saw, a logical possibility, they are not a natural possibility because this would violate the law of gravity.

Later, the notion of metaphysical supervenience will come into play. But before attending to that, and with this framework in place, let's see how Chalmers argues that consciousness does not logically supervene on physical/functional properties. He arrives at this conclusion via a thought experiment. He asks us to imagine his zombie twin, a being exactly like the real Chalmers, except that this creature completely lacks consciousness. Zombie Chalmers is a molecule for molecule copy of the real Chalmers. Not only is he physically identical, he is also functionally and behaviourally indistinguishable from the original.

Philosophical zombies come in at least three varieties: behaviourally, functionally or physically identical zombies. These three types are not distinct from one another, but rather form a continuum. Functionally identical beings are also behaviourally identical. Physically identical beings are also both functionally and behaviourally identical. A behaviourally identical zombie behaves in a way that is indistinguishable from the way we behave. A zombie who is functionally identical to us, not only behaves exactly like us, but also processes the same type of inputs from the environment, in the same way as us. These inputs have the same effects on its internal organization, and the zombie reacts 
by producing the same types of outputs (or behaviour) that we would. As the only way to explain how a creature could behave exactly like we do is to postulate that it functions cognitively like us, behavioural and functional zombies are closely connected. They are closely connected because we depend on behaviour (including verbal behaviour) in order to ascribe cognitive states to others. ${ }^{2}$

Returning to Zombie Chalmers, although it can tell us about its thoughts, its hopes and dreams, etc., it experiences no phenomenal feelings, there is nothing it is like to be Zombie Chalmers. ${ }^{3}$ Chalmers concedes that it is very doubtful that such a zombie could be naturally possible, that is, that it could be empirically possible in our actual world (Chalmers, 1996, p. 96). But Chalmers is untroubled by the fact that zombies could probably not exist in our world, because he maintains that the mere conceptual coherence of the notion is all he needs to establish his conclusion.

If we analyze the terms involved, and no contradictions appear, there is a natural assumption or intuition that points towards the logical possibility of the scenario. Chalmers thinks that it is open to the critic to suggest that our intuitions about the thought experiment are misleading us, but that the very fact that the scenario intuitively seems possible shifts the burden of explanation to the critic's side (Chalmers, 1996, p. 96). (Opponents sometimes critique thought experiments for their reliance on intuitions. Thought experiments are even dubbed “intuition pumps" by some (Dennett, 1999, p. 1).) Chalmers claims that, if a functionally isomorphic zombie is coherently conceivable, the facts about someone's functional organization do not logically entail conscious 
experience. Thus, he concludes that consciousness does not logically supervene on functional organization (Chalmers, 1996, p. 97).

According to Chalmers, the two main ways that conceivability arguments can be defeated are (1) if conceivability and possibility are pulled apart (as critics appealing to Kripke often claim) or (2) if a conceptual confusion or contradiction can be discerned in the description of the scenario (Chalmers, 1996, p. 99). We will first examine an objection that claims that the conceivability of zombies does not entail their possibility (or at least not in any important sense of possibility). Following this, we turn to objections that challenge the coherence of zombies, questioning whether or not zombies are properly conceivable. But for now, let us grant that zombie thought experiments are conceptually coherent.

A common strategy used to counter Chalmers' zombie thought experiment has been to draw a distinction between logical and metaphysical possibility and to insist that only if the possibility of zombies is established in the latter sense, is the argument a threat to functionalism.

The common objection to the possibility of zombies hinges on the notion of metaphysical identity, a notion developed by Kripke in his Naming and Necessity. Kripke draws a distinction between two different notions of identity: logical identity and metaphysical identity (Raymore, 2002, p. 3). Two things are logically identical if, by virtue of their meanings, it is impossible that they could be different things. Metaphysical identity depends not on meaning but on how our actual world turns out to be. The typical example of metaphysical identity is that holding between water and $\mathrm{H}_{2} \mathrm{O}$. It is 
conceivable that water might have turned out not to have the chemical constitution of two hydrogen atoms and one oxygen atom. But once we have discovered that it does have this constitution in our world (an a posteriori discovery), $\mathrm{H}_{2} \mathrm{O}$ will be identical to water in all possible worlds. Water and $\mathrm{H}_{2} \mathrm{O}$ are thus metaphysically identical.

Physicalists and functionalists who acknowledge that the possibility of zombies is a real problem, but who appeal to a posteriori necessities to counter it, claim that the identity relationship between consciousness and physical/functional processes is akin to that between water and $\mathrm{H}_{2} \mathrm{O}$ (Chalmers, 1999 , p. 4). Thus, while it is logically possible that an unconscious functionally identical zombie could exist, it just so happens that in our world, consciousness is physically instantiated. So, this functional phenomenon just is consciousness in all nomologically similar possible worlds. On this view, zombies are logically possible, but metaphysically impossible. Stated in terms of metaphysical supervenience, we get: “ $B$-properties supervene metaphysically on $A$-properties if it is metaphysically impossible for two situations/objects to have exactly the same $B$ properties without having the same $A$-properties" (Raymore, 2002, p. 4). Therefore, if conscious properties metaphysically supervene on physical/functional properties, any world that is identical to ours in its physical/functional properties, is ipso facto necessarily also identical in its conscious properties.

To counter the metaphysical identity move, Chalmers presents a two-dimensional framework of meaning and necessity, in which he draws a distinction between a concept's primary and secondary intensions (Chalmers, 1996, p. 56). For reasons that will 
soon become apparent as we turn to the second main way that conceivability arguments can be undone, his intricate response will not (and need not) concern us here.

Chalmers argues that because (a) zombies are conceivable and (b) conceivability implies possibility, (c) zombies are possible. If (c) zombies are possible, then (d) physical/functional facts do not logically entail facts about consciousness. Therefore, consciousness resists functional explanation. Chalmers concludes from this that functionalism about consciousness is false. The objection from a priori necessities focuses on (b), by challenging the move from conceivability to possibility. We believe that a much more effective strategy is to question the first premise (a). If it turns out that zombies are in fact inconceivable, zombie thought experiments are simply non-starters.

The philosophical zombie we are being asked to imagine, in Chalmers' thought experiment, is a composite of two ideas. The first is that of a human being who behaves and is built just like a normal person. This first idea must be combined with the idea that there is nothing "it is like" for this person on the "inside." In order to imagine a zombie, we must be able to put these two elements together (normal behaviour/internal organization and nothing "it is like inside") (Cottrell, 1999, p. 3). Can we really coherently hold these two ideas at once?

By stipulation, a zombie's sensory modalities all function without any accompanying subjective phenomenology. Yet, since it is functionally, and thus, behaviourally indistinguishable from us, this creature must be able to make the same type of fine-grained sensory discriminations that we can. It must process sensory information in the same way as us, by the same physical mechanisms, and this information about the 
world must be connected to behaviour in an appropriate way. Now imagine a zombie. Its sensory modalities don't provide the zombie with the subjective feelings that we normally experience, yet it can receive (and can use) all the information they provide to you and me. It can "smell" the bread in the toaster, "hear" its neighbour's television droning away, and if it notices that the room becomes too hot, it opens the window. There are scare quotes around the sensation terms because, by assumption, the zombie feels nothing when it detects these properties of the world. Is it really imaginable that it could make all these fine-grained distinctions and discriminations about the world without there being "something it is like" to make them?

Consider the following situation. Danny is two years old. He momentarily stops playing the game he's playing with his sister and lets out a "Brrrr...”, shaking as a shiver passes over him. Noticing this, his older sister Elena says “Danny a froid!", goes to the closet and gets Danny's coat. How are we to understand Elena's going to the closet to get her brother's coat rather than to the trunk where the toys are kept? The natural explanation is that she believed that his coat was in the closet - that she acted on the basis of an internal representation (or some other similar state playing the same role in her cognitive economy).

The only way we could explain the rich behaviour of the zombie would be to ascribe some type of similar representational states to it. Recall that the zombie is an exact functional isomorph of you or me. This means that this creature can report on and interpret information about its internal states. It can also consistently connect this information with both its behaviour and environment. We would not have the slightest 
inkling that this creature is not conscious and - neither would the zombie! It would believe itself to be conscious, to have beliefs and feelings just as we do. It would cry during sad films, blush and shy away from embarrassing situations, express pride in its work, react angrily when taunted, and describe in detail the intensity and quality of its rich and varied "experiences." It would believe that it is conscious, has feelings etc., on the basis of representations it has about itself; representations of the same kind regular humans must possess if their behaviour is to be understandable or be attributable to causes.

An objector would probably claim that there is a difference between the zombie's representing itself as having all these mental states and there being "something it is like" to have these representations. But then the onus of explanation shifts to the zombiephile who must explain how the creature could be cognitively identical to us and yet not be conscious. What exactly is the missing ingredient: what is it that makes consciousness more than all this?

If there is supposed to be a distinction between the zombie and me (it has no conscious experience), but this distinction corresponds to no describable difference, we have no reason to believe that it is a real distinction. This analysis may strike some as having a verificationist flavour to it. Indeed, there may be a tinge of verificationism in the air, but if this label be applied, it should be so in the weakest possible sense of the term. On this analysis, for a sentence to count as an assertion (or for it to be meaningful), there must be a difference between its being true or false. The distinction has to map some kind of difference in the world. Although it is not necessary that a sentence's truth-conditions 
be detectable or epistemically accessible, the difference that would make the sentence true rather than false (or vice-versa) must at least be stateable. In the case of zombie thought experiments, if this criterion cannot be met, that is, if we cannot even identify this difference, then we cannot suitably conceive of zombies. Not only can we not articulate the purported difference - we have no conception of what sort of thing could count as what the zombie is missing. And the same goes for the zombie itself: it could find no reason to doubt that it has feelings or is conscious because it could find no difference in its outward behaviour or even in its self-representing behaviour that could indicate that it is lacking something and is not actually feeling or conscious at all. If there is no describable, or even imaginable, difference between the zombie and a regular human, there is no reason to believe there is a real or meaningful distinction between zombies and conscious humans. If we cannot conceive of zombies, then it follows that they are not possible (not even in the sense of logical possibility).

There is a common fallacy that leads philosophers into believing that they can conceive of zombies. It rests on a failure to acknowledge a crucial distinction. BraddonMitchell draws a distinction between (i) conceiving that something is possible and (ii) directly conceiving of something (Braddon-Mitchell, 2003, p. 129). ${ }^{4}$ Now, that the possibility of zombies is conceivable does not mean that zombies are conceivable. ${ }^{5}$ Just as we can imagine imagining a square circle (or conceive of its possibility), we can conceive of conceiving of a zombie. But, being able to conceive of conceiving of a zombie (or of its possibility) is not the same as actually being able to conceive of one. ${ }^{6}$ In 
other words: "being able to conceive of conceiving $X$ does not entail that one can conceive of $X^{\prime \prime}$ (Brook and Raymont, Ch. 3, p. 33).

To imagine imagining something, we would at least have to be able to say in detail what features we would be combining. For example, I can imagine a circle and I can imagine a square. Then, I can imagine imagining a figure that has both these properties. But I cannot properly imagine a figure that combines both relevant features (both circularity and squareness). In the case of consciousness I can imagine a conscious being, say me, and I can imagine an unconscious being, say a rock, but I cannot imagine a being that is just like me in everyway minus consciousness. What is required for such an act of imagination to properly be carried out is that one could imagine the zombie in enough detail that if it somehow came to exist, it would actually have all the necessary features of a conscious human, minus the conscious aspects. Indeed, this is a tall order, but anything we imagine that does not at least meet this standard, is not sufficient to establish its conceivability. ${ }^{7}$

Zombie thought experiments aim to separate consciousness from cognitive functioning. The supposition that a zombie functions cognitively just as a real person and yet is not conscious, as we have seen, implies that something over and above cognition is required for consciousness to arise. We have found no candidate for the missing ingredient, and furthermore we have no reason to believe that we can coherently imagine such a separation. Dennett likens the attempt to separate consciousness from cognition to trying to separate physical health from the proper functioning of our various body parts. It is incoherent to try to imagine a person whose bodily functions are in perfect shape and 
yet is not also physically healthy. Just as health and proper bodily functioning cannot be separated, neither can consciousness and cognitive functioning (Dennett, 1995, p. 176).

If we are right, there is no way of coherently conceiving of a functionally identical zombie. If the zombist is to prove us wrong, she must provide a detailed account of how functionally identical zombies are conceivable. That is, she must show that the concept of a zombie is coherent, without falling into the conceptual confusions diagnosed above.

One way in which the zombie defender may attempt to establish the coherence of zombies, is by appealing to the notion of a pain-zombie. It does seem that we could imagine a pain-zombie. This is a zombie who cognitively processes all the same information related to pain that we do. It would use this information just as we do, seeking to avoid painful situations and acting just like us when we are in pain. But this zombie would do all of this without ever feeling pain. If we can imagine this, why couldn't we also imagine zombies who lack all consciousness?

We think it is open to someone who denies the conceivability of general zombies to concede that maybe we can imagine a being that lacks certain of our conscious states and yet behaves just like us. However, imagining a pain-zombie would not be enough we would also have to be able to generalize from this case and imagine a zombie who lacks all consciousness. There may seem to be no prima facie difficulty in generalizing. But now try to imagine a sound-zombie, who can distinguish between the timbre of a flute and that of a trumpet without there being anything it is like to "hear" this distinction. And imagine that it can also "see" and describe the hues of the falling leaves, without any 
accompanying phenomenal experience. We would have to generalize this pattern to include all our sensory modalities. It is far from clear to us that one could generalize the pain example in this way and still be correctly imagining the stipulated creature in all the relevant details. It seems to get harder with every sensory modality we subtract. "Harder, yes" claims the objector, "but not impossible." Well, we're not convinced (maybe our imaginative capacities need a tougher exercise regime?), but this still appears to be an objection with some, albeit limited, clout. $^{8}$

We hope to have shown that although zombie thought experiments cannot be ignored, their purported coherence and strength is, to say the least, highly questionable. Thus we conclude that these thought experiments do not discredit functionalist materialist approaches to consciousness and that there is no good reason to accept a radical dualism of the kind Chalmers espouses. ${ }^{9}$ The way is then open for us to adopt a functionalist materialist approach to the study of consciousness. Thus we can investigate the neural correlates of consciousness in terms of conscious functions.

\section{3 - Recent work on the NCCs}

The NCCs for many sensory modalities have been investigated. There is work on the NCCs of audition (Sabri et al., 2004), of food perception (Verhagen, 2006), and of thirst (Sewards \& Sewards, 2000), to name a few. However, most work has focused on the neural correlates of visual consciousness. The neural basis of vision been widely studied in non-human animals (Koch, 2004, p. 69). The many advances made in the neuroscience of visual processing in the last 25 years are mostly due to experiments on 
monkeys. Increasingly, efforts are being made to extending and applying this knowledge to humans (Heywood \& Milner, 2002, p. 241).

Milner and Goodale discovered that lesions to the ventral processing stream eliminate consciousness while lesions to the dorsal stream do not (Goodale 2004, p. 1161). ${ }^{10}$ The fact that damage to the ventral stream correlates with a loss of visual consciousness fuels their suggestion that the neural correlates of visual consciousness are to be found in that stream.

Some invoke very small scale neural activity. Hameroff speculates the NCCs are a series of quantum mechanical events in microtubules (which are subcellular structures) (Hameroff \& Penrose, 1996, p. 457). Others think NCCs are to be found by investigating large scale neural activity. Engel and Singer speculate that the neural correlates relevant to unified consciousness of global world- and self-models are to be found in the interactions between large groups of neurons synchronously firing in multiple frequency bands. They think that interactions between the different frequencies may be key to the NCCs (Engel \& Singer, 2001, p. 24). Tononi and Edelman believe that the neural substrates of consciousness are global patterns of highly complex activation resulting from interactions between large distributed groups of neurons (what they call "the dynamic core"). These patterns are found in the thalamocortical system, but are not restricted to this system (Tononi \& Edelman, 1998, p. 1849-1850). Llinás proposes that synchronous activity at $40 \mathrm{~Hz}$ in the thalamocortical system is the NCC (Llinás, 2003, p. 412). 
Some have speculated that the neural correlate of consciousness of unified objects is synchrony at $40 \mathrm{~Hz}$, recorded mainly over occipital lobes (Rodriguez et al., 1999, p. 431; Keil et al., 1999, p. 7156; Tallon-Baudry et al., 1996, p. 4246; Revonsuo, 2006, p. 280). Tong et al. propose that the neural correlate for the consciousness of faces is increased activity in the fusiform face area (FFA) (Tong et al., 1998, p. 758).

In a popular study using the binocular rivalry paradigm (described in Chapter 2), Sheinberg \& Logothetis (1997) were able to dissociate two neural systems in the monkey's visual cortex. They suggest that the NCCs of visual consciousness are in IT/STS (inferotemporal cortex and superior temporal sulcus), and not in the early visual cortex (areas V1, V2, V4, and MT) (Sheinberg \& Logothetis, 1997, p. 3408). Fries et al. found that synchronous action of V1 neurons in the awake cat correlates to the winning stimulus in binocular rivalry (Fries et al., 1997, p. 12703). Lumer et al. propose that the neural correlate of the perceptual transitions experienced by humans in binocular rivalry is activity in the parietal and frontal regions (Lumer et al., 1998, p. 1930).

Researchers such as Morris et al. (1999) have used visual masking to compare activity between brain areas correlating with reflexively conscious and non-reflexively conscious processing. ${ }^{11}$ They found increased activity in the midbrain and amygdala regions in the masked condition correlating to the non-reflexive consciousness of visual stimuli (Morris et al., 1999, p. 1680).

Rees compared many studies investigating NCCs in the parietal and prefrontal cortex areas. His meta-analysis singles out two areas (superior parietal and dorsolateral prefrontal cortex) whose activity, despite the different stimuli and experimental 
paradigms used by researchers, seems to correlate with visual consciousness (Rees, 2004, p. 1183). Rees speculates that in addition to activity in functionally specific areas of the visual cortex (such as V1 for brightness and contrast, or V5/MT for motion awareness), activity in prefrontal cortex and/or parietal cortex may also be needed for consciousness (Rees, 2004, p. 1184). This is consistent with Crick \& Koch's (1995) hypothesis that the neural correlates of visual consciousness do not directly involve neurons in V1, but are to be found in activity involving visual areas higher up in the processing stream (especially in the ventral stream), and the prefrontal cortex (Crick \& Koch, 1995, p. 375; Crick \& Koch, 2003, p. 120).

As witnessed by the history of recent work on NCCs, many different sorts of NCCs have been proposed, and many different brain areas have been suggested as their seat. Indeed, Revonsuo notes that nearly all cortical lobes have been proposed as the locations of the NCCs (Revonsuo, 2001, p. 5).

Identifying the neural correlates of consciousness is one thing. But, explaining why this activity correlates to a particular conscious state is another. The goal is to know what it is about the correlated activity that makes conscious states conscious. This general problem of understanding the relations between physical processes and consciousness is an example of what Levine called an "explanatory gap" (Levine, 1983, p. 354). At present, this problem seems intractable. Future progress in NCC research should aim to explain how the correlated neural activity actually implements or realizes consciousness by specifying the neural mechanisms involved and their functioning. 


\section{$1.4-\underline{\text { What we hope to contribute }}$}

We aim to show that paradigms involving visual masking of illusory primes provide a method for comparing brain activity which correlates with different levels of conscious unity. Thus, masking paradigms can be used to investigate the neural correlates of specific functions of unified consciousness. By proposing a protocol which is more specific from both functional and structural perspectives when compared to existing studies of the neural correlates of unified consciousness, we hope to contribute a methodology for focusing in on the neural mechanisms of unified consciousness. More specifically, our analysis of the work done by researchers attempting to discover the NCC for the function of "individual object unity" shows that they fail to distinguish this function from another related, but different, function of unified consciousness (the "multiple object unity" function). Building on recent experimental work done in another context, we show how these two functions can be isolated and we suggest further experimental interventions aimed at pinning down the structural and functional properties of the mechanisms underlying these functions.

\section{5 - Short summary of upcoming chapters}

In Chapter 2, we progressively explain what is meant by "neural correlates of consciousness." We discuss how they are found and some methodological problems. Then, we look at the main methodological challenges involved in mechanistic explanations. We set out the framework from which we will examine/evaluate different approaches to NCCs as to how they fare at bringing us closer to the neural mechanisms 
underlying the unity of consciousness. While the latter half of Chapter 2 contains reflections on what could be called the "philosophy of science" of NCC research, Chapter 3 moves from these meta-level considerations to the phenomenon itself. After introducing the unity of consciousness and some of the particular challenges it poses, we examine "local" approaches to the NCCs to see if they could help uncover the mechanisms of unified consciousness. In Chapter 4, we consider "global" approaches to the neural correlates of unity. We end our project with what we hope will be our main contribution: a protocol for discovering the neural correlates of the "individual object unity" and "multiple object unity" functions. The aim of this proposed methodology is to zero in on the neural mechanisms underlying these functions of unified consciousness. 


\section{On the Search for the Neural Correlates of Unified Consciousness}

\section{Chapter 2}

\section{1 - Introduction}

In this chapter we progressively explain what is meant by "neural correlate of consciousness" (NCC). We discuss how they are discovered and methodological problems (concerning lesion studies and ascriptions of consciousness). Then the role of NCCs in the study of consciousness is considered. We discuss mechanistic explanation and methodological problems related to it. This will provide the framework from which we examine and evaluate different approaches to the NCCs in relation to the promise they show of revealing the mechanisms of unified forms of consciousness. Before getting to the question of what a "neural correlate" of consciousness is, let's clarify what we mean by "consciousness."

\section{2 - General types of consciousness}

Let's begin with a very general type of consciousness: “creature consciousness." Creature consciousness could be described as a general state of alertness. To be conscious in this sense is to be conscious as opposed to being in a dreamless sleep or a coma. Our second and third types of consciousness, "consciousness of the world," and "state consciousness" are more specific in that they are either about items in the world or about our conscious states. Although we must be "creature conscious" in order to have "consciousness of the world" or "state consciousness" we focus on the latter two types of 
consciousness. The distinction between these two types of consciousness will be important to our project so it is worth taking a moment to discuss it here.

To be conscious in the sense of "consciousness of the world" is to be conscious of an item without also being conscious of the state in virtue of which one is conscious of that item. For example, I can be conscious of a table without being conscious of the state in virtue of which I am conscious of the table. When I am conscious of my experience of seeing the table, I am conscious in the sense of "state consciousness." In this third sense of consciousness, I am conscious of one of my conscious states (such as my mental state which represents the table) or of aspects of one of my conscious states. The difference between consciousness of the world and state consciousness is the difference between being conscious of $X$ and being conscious of being conscious of $X$. To illustrate this difference, consider someone driving from New Brunswick to Nova Scotia. ${ }^{12}$ Having driven this route regularly for many years our driver could be surprised to find herself having arrived at her destination without having really noticed the last part of the drive. She may feel that she drove on "automatic pilot." In this case, she would have been conscious of the road (consciousness of the world) without being conscious of being conscious of the road (without consciousness of her own conscious states or state consciousness). Had she been looking at the road's reddish colour and mentally contrasting it with the more common black, she would have been conscious of aspects of her conscious states (state consciousness).

State consciousness can be considered as a type of self-consciousness because it involves reflecting on our experience or turning our attention "inwards" towards our own 
mind and its "contents." (Conscious "contents" are the intentional objects or items that one's conscious states represent or are about.) Some of the things such introspection can reveal are our mental states (perceptual states, thoughts, beliefs, emotions, desires...), properties of our mental states ("what it is like" to have them, how intense they are, what they are about - their contents...), and our selves. We will sometimes refer to state consciousness as "reflexive" consciousness and to consciousness of the world as "nonreflexive" consciousness.

When NCC researchers talk of consciousness, they are typically referring to consciousness of the world. However, in the majority of experiments involving humans, participants are asked to report on aspects of their conscious states (verbally or through some other behaviour). Thus, because the participants must reflect on their own experience in order to issue reports, they are reporting about consciousness of their own states. NCC researchers are generally quite hostile to the idea of basing a science on introspection (reflexive consciousness) (Hohwy, 2007, p. 465). Thus, it is ironic that they find themselves depending on it in this way. (Experiments involving masked priming are an exception since, as we discuss later in this chapter, they involve consciousness of the world without also necessarily involving consciousness of one's own states. Thus, they don't necessarily involve introspection.)

\section{3 - Will what we learn from visual consciousness generalize to other modalities?}

In Chapter 1, we mentioned that although the NCCs for different sensory modalities have been investigated, most work has been done studying visual 
consciousness. Koch thinks it likely that the different aspects of conscious experience (vision, pain, smell, etc.) function according to a unique underlying mechanism or a few such mechanisms. He bases his assumption that similar neural events and circuits are responsible for the different aspects of consciousness on the way that natural selection operates (Koch, 2004, p. 15). We take it that he is referring to the idea that natural selection tends to be conservative, in the sense that rather than coming up with completely new mechanisms, it functions by modifying previously successful ones, thus “spending" as little as possible. If this is true, understanding the neural basis of one modality should aid in understanding the others. ${ }^{13}$ Accordingly, our focus will be on the neural correlates of visual consciousness and on the neural correlates of unified forms of visual consciousness. Visual consciousness is a type of phenomenal consciousness. A conscious state is phenomenally conscious if there is "something it is like" to experience it.

\section{$2.4-$ What is a neural correlate of consciousness?}

To answer the question "What is a neural correlate of consciousness?" we start with a general definition taken from what is sure to become a standard reference about the conceptual issues in NCC research - Chalmers' article of the same name. A neural correlate of consciousness is a "specific system in the brain whose activity correlates directly with states of conscious experience" (Chalmers, 2000, p. 18).

Not all neural activity that accompanies a particular conscious state is considered part of that conscious state's neural correlate. Researchers typically distinguish between 
the "minimal" NCC (or "core" NCC in Chalmers" vocabulary) for a particular conscious percept and the more global NCC of this percept which includes background conditions. Saying that an NCC should only contain the core processes that suffice for the conscious state it is paired to means that we can "bracket" background or enabling conditions that may also be necessary for consciousness (Chalmers, 2000, p. 25). For example, in order to experience $y$, it may be necessary that the subject also be in a particular overall state (such as creature consciousness or being awake). This background state may enable $y$ without being directly tied to it. Putting such background or enabling conditions aside allows for a focus on the NCCs that are more directly involved in the experience of $y$. We can sharpen our definition by adding this idea to it: an NCC will be a state of the minimal neural system which is sufficient for a corresponding conscious state (Chalmers, 2000, p. 26). The neural system is qualified as minimal to reflect the fact that only the "core" NCC is being sought. The minimal neural system is the system that makes this particular experience, the experience it is. We would know that we discovered the minimal neural system correlating to a particular percept $x$ if selectively removing (or somehow deactivating) the system cancels the experience of $x$ while leaving all other experiences unaltered. In practice, however, the dissociation of such a minimal system from other systems faces methodological problems. (We'll discuss some of these problems shortly.)

The reason it is not a requirement that, as well as being sufficient, the minimal neural system also be necessary for the corresponding conscious state, is that we cannot rule out on a priori grounds that to each conscious state there will only be one NCC. There may turn out to be more than one neural system that correlates with the same 
conscious experience (Chalmers, 2000, p. 25). The reason there might be more than one NCC for the same individual conscious state is that one conscious state could be realized in different ways. Conscious states are said to have "multiple realizability" because different neural systems or different forms of activity could all realize the same conscious state. And one neural state cannot be necessary to a conscious state if there's another neural state that is sufficient, on its own, for this conscious state.

\section{5 - “Content” NCCs}

Let us now look at an influential version of minimal NCCs - the NCCs for content. In the case of NCCs for background states of consciousness (e.g., general states of consciousness like creature consciousness, being awake, dreaming, etc.), it may be enough that a neural system's overall state correlates with the background state. For example, Koch reviews evidence that there is a "cholinergic enabling system," made up of neurons that release the neuromodulator acetylcholine. The activity of these cells, located all over the cortex, the thalamus, and the basal ganglia seems necessary for consciousness without however being sufficient for any particular percept (Koch, 2004, p. 91). While some seek to pin down the NCCs of background states of consciousness, the most interesting work is being done trying to find the neural correlates for specific conscious contents. One could look to find the NCCs for different types of contents (visual, auditory, tactile). There may turn out to be different kinds of NCCs that match up with these different types of conscious content. But, neuroscientists usually look for the 
NCCs of specific subtypes of contents within a modality (like horizontal gratings in visual consciousness or the sound of high $\mathrm{C}$ played on a piano in auditory consciousness). Many general states of the nervous system could be said to correlate with a background state of consciousness such as wakefulness. But the demands on an NCC for a specific conscious experience (e.g., seeing a moving dot) are more stringent. Specific contents of conscious experience must systematically correlate with specific states of a neural system. As Chalmers puts it, "where [a specific conscious] content is concerned, not any neural state will do. We require that the content of the neural state in question must match the content of consciousness" (italics in original) (Chalmers, 2000, p. 23). Finding systematic correlations between the "content" of neural states and the content of conscious experience is the way to arrive at NCCs for specific contents of consciousness or "content NCCs." However, rather than require that the content of a neural state "matches" the content of consciousness, we should require that it correlates with this content. Matching is too high a demand because it implies the requirement that a certain isomorphism between the neural state and the conscious state obtain.

Consider the visual experience of a homogenous blue sky over a homogenous expanse of desert sand. The requirement of isomorphism would mean that for a neural state to count as this conscious state's neural correlate, there has to be structural similarity between the neural state and the conscious state. For example, upon presentation of the desert scene, perhaps two different populations of neurons exhibiting a marked increase in firing rate could be taken to match the two different homogenous regions in the visual experience. 
How could this matching work? Perhaps by way of spatial isomorphism? A spatial isomorphism constraint would imply that the visual scene of an unbroken blue sky over a homogenous expanse of desert sand (the content) would have the same spatial layout in the neural substrate (the vehicle) which underlies this experience. That is, whatever somehow codes for the sky in the brain would be located spatially above whatever codes for the sand. Note that some parts of the sensorimotor and visual cortex code in a topographically isomorphic way. For example, in the primary visual cortex (V1), all areas are precisely mapped topographically to corresponding areas in the retina. With damage to V1 comes a loss of conscious vision in the corresponding part of the visual field, while non-reflexively conscious detection of object location and movement can remain at least partially intact (some consciousness of the world can remain) (Koch, 2004 , p. 220). But to take topographical mapping as a general rule would fly in the face of what we know about the distributed nature of perceptual encoding. It is widely believed by neuroscientists that perceptual information (and other information relevant to cognitive functions) is distributed across different areas of the brain (Engel, 2003, p. 32). Typical visual experience is made up of scenes which are much more complex than two homogeneous zones. In your experience of a single wheat stalk, with an isomorphism constraint, there would have to be some structural similarity between the wheat stalk and some neural state $N$. But, what if your experience is of a thousand wheat stalks? Would you now have a larger complex neural state equivalent to a thousand smaller $N$ s? The distributed nature of perceptual encoding (the fact that different features appear to be processed in different brain areas) makes spatial isomorphism seem unlikely. 
Chalmers clearly believes that the definition he gives of neural correlates for conscious contents does not require a spatial isomorphism between conscious states and neural states (Chalmers, 2000, p. 37). And yet he often speaks in terms of "matching" conscious states to neural states. He even sometimes talks of "mirroring”: “...there is reason to expect that conscious contents will be mirrored by the contents of a neural representational system at some level of abstraction" (Chalmers, 2000, p. 35). He qualifies the "mirroring" as being "at some level of abstraction" in order to avoid requiring a spatial isomorphism.

But, if the isomorphism isn't spatial what could it mean for a neural state to "match" a conscious state? And what about forms of consciousness other than visual consciousness? Considering them further brings out the implausibility of isomorphism. What would it mean for a neural state to structurally resemble the sound of an E Minor chord, the smell of cumin, or the feel of silk? To talk of matching is to run the risk of confusing attributes of the content (like structural aspects of the conscious experience) for attributes of the vehicle (like structural features of the neural substrate which encodes the contents). Thus, we'll restrict ourselves to using the more neutral notion of "correlation." In this way we avoid pre-judging the structural characteristics of NCCs which ultimately is an empirical issue.

Now for a more complete notion of a neural correlate of consciousness. The following definition of a "content NCC" reflects much work currently being done by neuroscientists: "An NCC (for content) is a minimal neural representational system N such that representation of a content in $\mathrm{N}$ is sufficient, under conditions $\mathrm{C}$, for 
representation of that content in consciousness" (Chalmers, 2000, p. 31). (Note that this definition involves more than mere correlation. It states that the minimal system is what we want and that this minimal system must be sufficient for the conscious content it correlates with.)

It follows from this definition, that if we are to find the NCC for a specific conscious experience we need to be able to correlate this experience with the "representational content" of a neural system. It is common in philosophy of mind to speak of conscious content. For example, my visual experience as I look out the window can represent (or be about) a grey squirrel unearthing a tulip bulb. The squirrel and the illicitly obtained bulb are two intentional objects or contents of my present conscious state. But, how are we to understand the notion of a neural representational content? In general one can think of the "representational content" of a neuron as the activity of the neuron that correlates with a specific stimulus or conscious content. The term "neural coding activity" is more appropriate - there are good reasons for eschewing talk of "neural representational content" in relation to neural systems believed to be NCCs. First, by using "representational" or any other cognate of "representation" we run the risk of confusing attributes of conscious contents (such as possessing intentionality) with attributes of their vehicles (the neural substrates and their activities) which encode these contents. This is the same sort of danger involved in talking about "matching" and “mirroring” between neural and conscious states. Second, looking for neural activity which correlates with conscious contents is what researchers actually do. 
There could be a neuron which fires vigorously whenever bulbous objects are consciously experienced while other percepts fail to alter its base firing rate. In this case the cell's coding activity (its "content") could be said to correspond to a specific conscious content (bulbous percepts). The "representational content" of a neural system would be made up of some of the coding activities of the neurons which comprise it. We'll see, in Chapters 3 and 4, that neuroscientists differ as to the scale of neural coding activities (and underlying levels of neural organization) they consider relevant to the NCCs.

Do neuroscientists actually operate with the notion of content NCCs? Yes. We find an example in the definition that Koch uses to guide his own neuroscientific research. He states that an NCC is the "minimal set of neural events jointly sufficient for a specific conscious experience (given the appropriate enabling conditions)" (italics in original) (Koch, 2004, p. 97). Here "specific conscious experience" is equivalent to a (specific) content of consciousness. Koch's insistence that the NCC be equated with the minimal set of neural events in contrast to the background or enabling factors shows that he embraces the idea that minimal NCCs should be sought. In addition, the requirement that the neural events be sufficient for the conscious content they correlate with is explicit in this definition. ${ }^{14}$ 
2.6 - How to find the NCCs: general strategies, methodological problems (lesion studies, ascriptions of consciousness)

We've talked about how researchers draw a distinction between total NCCs and minimal NCCs. How, in practice, can we distinguish minimal NCCs from systems linked to them? Studying normal cases is not enough. That is, looking for correlations in a normally functioning brain in a normal environment (restricted to everyday stimuli) will not do. The reason investigating normal cases is not enough is that in normal cases the various systems involved will not be dissociated from each other since they'll be linked by part/whole relations.

Between two normal situations in which a particular stimulus is experienced, there are countless variations in the background conditions. Knowing this, we could, for instance, try to compare brain activity in situations where the background conditions vary (in a noisy room, in a walk-in refrigerator, on a windy beach) while trying to keep the presentation of the target stimulus constant (by showing it at the same angle and distance from the subject, controlling the lighting conditions, etc.). Because the target stimulus is kept somewhat invariant, the idea is that we could look for neural activity that is invariant or common to the different situations. Maybe this invariant activity would be the neural correlate for the target conscious stimulus. The problem with this strategy is that many background states of consciousness (like being awake) will also be common to the different situations. There would be no way to tell which invariant neural activity is part of the NCC for the target stimulus. We couldn't isolate the minimal neural system from other systems that are merely linked to it. Thus, even if we controlled aspects of the target 
stimulus' presentation, studying it in the context of otherwise normal cases would not be enough for dissociating the systems involved.

Let's say the activities of neural areas $x, y$, and $z$ all correlate with a conscious content. Is $y$ active because it is involved in the background state of being awake? Of the whole $(y+x+z)$, we want to discover which part or parts are specifically relevant to the target conscious content. How can we figure out if these areas are really part of the minimal system and are crucial to the conscious content? To yield dissociations between neural systems one must also look for correlations which hold across unusual cases (Chalmers, 2000, p. 27).

One unusual case which can produce dissociations involves directly stimulating a normal brain (with electrodes for example (see Koch, 2004, p. 99)). If by stimulating a certain brain area one could systematically produce the same conscious experience while showing that stimulation of another area didn't produce the experience, this would suggest that the first area is the NCC (or part of the NCC) of that experience while the second is not.

Another unusual case is produced by using transcranial magnetic stimulation. With transcranial magnetic stimulation (TMS), localized activity under an electrode is temporarily disrupted by a strong magnetic field. To see if a particular brain area is involved in the consciousness of a specific content, we can use TMS to temporarily disable this area. If the subject no longer reports experiencing the content when TMS is applied, this may be a sign that the area's normal functioning contributes to consciousness of the content. But, it could be that the TMS knocked out the reportability 
while leaving the experience of the content unaffected. To control for this we could present a variety of stimuli and ask the subject to report their consciousness of these contents. If they can report consciousness of many other contents when TMS is applied but not report consciousness of the initial content, then TMS was probably selectively knocking out the content rather than the reportability. If the subject cannot report consciousness of the other stimuli (contents), then the area could be involved in more general mechanisms of reportability or be involved in consciousness more generally (rather than only with the specific target content).

It is also possible that the area's normal activity is not needed for consciousness of the content. Perhaps this area merely reacts to the target stimulus in the way that a light goes on when a switch is flicked. How could we control for this? By leaving that area alone and knocking out related areas, we can check if the subject can still report the content. If they cannot, it is possible that the first area was merely acting like a light going off but that another related system is the crucial one for the target stimulus. ${ }^{15}$

Dissociations are also found in a third type of case: brain lesions causing abnormal functioning. Studying patients with lesions is a popular means to reach conclusions about NCCs. (Note that the following example concerns the neural correlates of consciousness more generally, not the NCCs for specific contents.) Milner and Goodale discovered that lesions to the ventral stream eliminate consciousness while lesions to the dorsal stream do not (Goodale, 2004, p. 1161). (The ventral stream is associated with visual recognition while the dorsal stream is associated with motor response.) The fact that damage to the ventral stream correlates with a loss of visual 
consciousness suggests that the NCC is located in that stream. Since damage to the dorsal stream correlates with relatively normal visual consciousness, this stream does not appear to be a candidate for the location of the NCC. Such conclusions depend on the implicit premise that an NCC should correlate across abnormal cases in much the same way that it does across normal cases (Chalmers, 2000, p. 28).

Chalmers is sceptical about the use of lesion studies in determining the location of NCCs and warns against the methodological dangers involved in their use. He gives reasons why a requirement that an NCC correlate over abnormal cases would be too strong and methodologically risky. First, a lesion in a part of the brain that plays a role in the background or "total" NCC of a percept could destroy the correlation in the same way that serious damage to the lungs can stop the brain's operation or that an intact retina is necessary to normal visual perception. Second, lesions in parts of the brain (other than in the minimal NCC) could change brain architecture in a way that what was once an NCC no longer functions like one. If an NCC must play the right functional role in order to support consciousness, damage to the neural system could alter its role within the system, thereby destroying the correlation. Third, lesions in the NCC could also change brain architecture in a way that what was the NCC no longer functions like one and yet the conscious experience it is paired to remains normal. When a brain area is damaged or excised and a certain conscious experience remains more or less normal, it is commonly concluded that this area cannot contain the NCC of this experience. However, drawing this conclusion is dangerous for the following reason. Though not explicitly mentioned by Chalmers, it seems plausible that, in these cases, the brain's plasticity allows that 
certain other systems compensate to some extent for damage in the original NCC. Let's say $x$ is the NCC of conscious state $y$, and that $x$ sustains serious damage. It is possible that through reorganization, neural systems $u$ and $w$ take on the role previously played by $x$. Thus the role that $x$ plays as an NCC in a healthy organism could shift to a different neural system in a damaged organism. Such reorganization is not instantaneous. It takes time and learning for reorganization to happen. ${ }^{16}$ The above considerations do not make lesions studies irrelevant to NCC research. They can still provide evidence regarding NCC location, even if this evidence is indirect. Chalmers' point is a cautionary one. It is a reminder that prudence is in order when drawing conclusions about NCCs on the basis of lesion studies.

A fourth type of unusual case which can reveal dissociations is presenting a normal brain with unusual stimuli which aren't normally experienced in a regular environment. The stimuli used in the binocular rivalry paradigm are good examples. In this experimental paradigm two different stimuli are presented to the left and right eyes. These stimuli are incongruent such that they cannot be fused into a coherent percept. A normal human subject experiences the two stimuli alternately but never at the same time. While one stimulus is experienced (the winning stimulus), the other is suppressed (the suppressed stimulus). In a famous study using these unusual stimuli, Sheinberg \& Logothetis (1997) were able to dissociate two neural systems in the monkey's visual cortex. They found that cells in early visual cortex (areas V1, V2, V4, and MT) responded to both winning and suppressed stimulation. But cells in areas IT (inferotemporal cortex) and STS (superior temporal sulcus) responded only to the 
winning stimulation. They take this to suggest that the neural correlates of visual consciousness are in IT/STS and not in the early visual cortex (Sheinberg \& Logothetis, 1997, p. 3408). Recall the distinction we drew at the beginning of this chapter between consciousness of the world and consciousness of one's own states. Here, Sheinberg and Logothetis are using "consciousness" to refer to consciousness of a stimulus (consciousness of the world), not to consciousness of being conscious of a stimulus (state consciousness).

We should note here that, even though a human subject could not report consciousness of the suppressed stimulus, the suppressed stimulus in binocular rivalry experiments may have some cognitive effects which are observable in the subject's behaviour. These effects showing that information from the suppressed/unreportable stimulus was cognitively processed would indicate that the subject was actually also conscious of the suppressed stimulus in the sense of consciousness of the world (albeit to a much lesser degree than she was conscious of the winning stimulus). Our fifth example of an unusual situation used to reveal dissociations actually focuses on this sort of cognitive or conscious activity of which the subject is unaware.

In masked priming paradigms the presentation of a stimulus (the "prime") is quickly followed by the brief presentation of a "mask" (usually a random pattern or some other stimulus different from the prime). If a mask is presented a few $10-100 \mathrm{~ms}$ after the presentation of a stimulus, the participant cannot report consciousness of the stimulus. (Participants are not conscious of being conscious of either the mask itself or of the masked stimulus.) However, what is interesting is that participants may show signs that 
the masked stimulus lead to cognitive processing even though they were unaware of this processing. Indeed, although the subject could not report being conscious of the stimulus, because the stimulus had behavioural effects (which indicate cognitive processing), we can infer that the participant was in fact conscious of the stimulus in the sense of "consciousness of the world."

The first important point to be made in relation to the masked priming paradigm is that it provides the experimental means for marking the distinction between consciousness of the world and consciousness of one's own states. It brings to light the cleavage that can exist between being conscious of a stimulus and being conscious of being conscious of a stimulus. Later, when we investigate the neural correlates of unified consciousness, this distinction will be key to the dissociation of the neural correlates for two different functions of unity. ${ }^{17}$

We said that if behavioural effects show that perceptual information about the stimulus (information about the world) was used to influence behaviour, then we can infer that the subject was conscious of the stimulus. How can such effects on behaviour be measured? Effects due to the processing of information about a masked stimulus can be measured on a subsequent task (a task immediately following the presentation of the masked stimulus). One such task, for instance, is having to decide, as quickly as possible, whether or not a string of letters comprises a word (Marcel, 1983, p. 219). If two words which share a semantic or associative relation are presented successively, the reaction time for identifying the second word is faster. For example, if "butter" follows "bread" it is usually classified faster than if it follows an unrelated word such as "nurse". Thus, if a 
similar effect (a "priming effect") can be measured following the presentation of a masked word, we can infer that the subject had non-reflexive consciousness of the masked word. (In this example, "bread" and "nurse" are the primes and "butter" is the target for the task.)

The second important point which emerges from this discussion is that ascriptions of state consciousness and consciousness of the world are made on the basis of different sorts of behaviours. When the subject is able to report their consciousness of a stimulus it is an indication that they are conscious of their own conscious states. Availability for report implies state consciousness. When unreportable stimuli nevertheless show signs of having been cognitively processed resulting in controlling or influencing behaviour, this indicates that the subject was conscious in the sense of consciousness of the world. Availability for report and availability for control of behaviour are two indicators of consciousness. Chalmers sees "global availability" (availability for report and availability for control) as being central to the ascriptions of consciousness in experimental work on NCCs (Chalmers, 1998, p. 4). ${ }^{18}$

Since we still lack instruments that can directly measure consciousness, we must rely on indirect behavioural evidence. For example, the most widely used indicator of consciousness, at least in the case of humans, is the capacity for verbally reporting the contents of consciousness. Indeed, when one turns to one's own experience, there is a correlation between many of our conscious states and our ability to report them. Since we do not actually have to report all we are conscious of in order to be conscious of it, it is 
fitting to consider availability to report as the relevant index of consciousness (Chalmers, 1998, p. 3).

Information is available for report when the subject could report being conscious of it either verbally or through some other behaviour. The subject could, for example, push a button to signal consciousness of a stimulus. When information is available to verbal report, it is also usually available to other motor systems. Availability for report is sufficient for availability guiding behaviour. Availability for guiding behaviour is necessary for reportability. But availability for guiding behaviour is not sufficient for reportability.

Limiting the ascription of consciousness to subjects that produce verbal reports about their experiences would be to neglect one the richest sources of data about the NCCs: experiments involving animals. The main reason for using animals is that certain studies involve invasive measures (e.g., inserting electrodes into the brain to monitor the activity of neurons) that cannot ethically be practiced on humans. ${ }^{19}$ Experiments on nonhuman animals require even more indirect measurements of consciousness, but the basic idea of having information available for global control seems to be widely assumed to be the relevant index. For example, in the Logothetis experiments cited above, monkeys were taught to pull a lever to indicate whether they perceive one bistable percept rather than the other.

One may wonder what the difference is between a monkey's pulling of the lever and a human's pushing a button to signal consciousness of one stimulus rather than another. A human can be asked to report consciousness of a stimulus by pushing a button. 
This would indicate that the subject is conscious of their own conscious states. Wasn't the monkeys' moving of the lever similarly a report about their conscious states implying consciousness of being conscious of the world? In the case of animals, the behaviour signaling consciousness of a percept could simply be the result of having learned to associate certain responses with rewards. For example, monkeys were taught to associate the sight of one of the stimuli with pulling the lever to the left because this behaviour was reinforced by a reward (fruit juice). Thus, a monkey's pulling of a lever may not be a report about its conscious states, but rather a learned association to stimuli without consciousness of being conscious of these stimuli. So although it is possible that the monkeys are in fact actually conscious of their own states, because their lever-pulling behaviours could be conditioned responses, we cannot infer that their actions reflect state consciousness. In the case of humans, we can guard against effects of such learned associations by simply asking participants what they are experiencing - there's no need to train them to respond correctly.

Whether with humans or other animals, the only index of consciousness we have is behaviour (verbal or other). Is this problematic? The fact that we can only indirectly measure consciousness (in others) leads to the following question: How can we know when a system is in a given conscious state? This is the "problem of other minds" and of the "privacy" of consciousness. Since neuroscientists attempt to find correlations between specific conscious states and neural systems, they must have a way to know what conscious state a subject is having. Some may be tempted to ask the further question, "And if we cannot reliably know what particular conscious state a system is in, how can 
we know it is conscious at all?" We refer those who have this temptation and any accompanying worries about zombies to Chapter 1 .

As diagnosed by Brook and Raymont, the temptation to construe consciousness as essentially private and inaccessible to others comes from the fact that there can be a slippage between one's conscious states and one's behaviour (Brook \& Raymont, forthcoming, Ch. 4, p. 3). It is a normal aspect of our mental lives that we keep some of our thoughts and feelings to ourselves (or that we sometimes let on that we feel/think differently than we actually do). But to infer from the fact that we sometimes dissociate our conscious states from our behaviour to the idea that behaviour is not generally a trustworthy guide to others' conscious states is an overgeneralization. Although it may not be infallible in all situations, behaviour (including verbal behaviour) is the best (and only!) guide we have to the conscious states of others. Of course one cannot be completely sure that a participant in a study is actually experiencing what they report. They could, out of maliciousness, report having experience $\mathrm{n}$ when they know that reporting experience $m$ would be the honest response. While these problems don't keep neuroscientists up at night, they do remind us that, as discussed in Chapter 1, ascriptions of consciousness depend on observable behaviour. Does this imply that neuroscientists make an epistemologically suspect "leap of faith" whenever they ascribe conscious states to others on the basis of behaviour? At least in cases involving awake humans, they are welcome to the same amount of certainty you and I share in our daily lives. So, neuroscientists are no worse off than you are when you ascribe conscious states to me, to 
your family members, or to anyone. Whether in everyday life or in the laboratory, behaviour is the only indicator we have of others' consciousness.

Because they show signs of manipulating perceptual information to guide their behaviour, we believe there is little room for doubting that the awake animals used in NCC research have some form of consciousness of the world. Due to the behavioural similarities between monkeys and humans, our simian cousins are often used in experiments that attempt to uncover the mechanisms of consciousness. The fact that monkeys also share many similarities with humans in terms of development and brain structure is an additional reason not to doubt that their behaviour signals consciousness (Koch, 2004, p. 13). However, we can rightly entertain these doubts when the animals are anaesthetized. ${ }^{20}$ High doses of anaesthetic probably affect the animals' phenomenal consciousness as they do in the case of humans.

We now have a better idea of what NCCs are and how to look for them. But before getting to NCCs themselves (in Chapter 3), we take a step back to examine the question of what type of explanation we want in a study of consciousness. We look at mechanistic explanation and some of the methodological problems it poses.

\section{7 - The role of NCCs in consciousness studies}

The main impetus behind the NCC search is the hope that their discovery will lead the way to a better understanding of the relations between neural states and conscious states (the famous mind-body problem). Identifying the NCCs is not the final step in the study of consciousness. Saying that two states "correlate" is not a satisfying 
explanation of the relation between neural states and conscious states. If we discovered that a neural state $N$ somehow co-varied with a conscious state $S$, we could say that $N$ correlates with $S$. For, example, we could discover that every time $S$ is present, $N$ is present as well and vice-versa. However, the discovery of such a mere correlation on its own would be uninformative for it doesn't explain the relations between $N$ and $S$. After the NCCs are identified, it must be explained why the correlation holds.

There are a variety of relations which could yield NCCs. First, NCCs could result from causal relations between neural and conscious states. Discovering that $N$ causes $S$ could explain the relation between the two states. Note that this explanation would require a notion of causation different from the one used in physics (which necessitates the exchange of a quantity of energy). Second, constitutive relationships between neural and conscious states could yield NCCs. That is, perhaps we will discover how $S$ is realized (or implemented) by $N$ because of $N$ 's particular constitution (what $N$ is made of and how it is structured). Third, emergent relations between neural and conscious states could result in NCCs. Maybe conscious states will turn out to be higher level properties that arise or emerge from lower-level neural properties and processes. Whether we're talking of causal, constitutive, or emergent relations between neural states and conscious states, the explanation we want must explain how the relation works. 
2.8 - Mechanistic explanation: methodological issues - decomposing mechanisms and levels of analysis

While sciences like physics may have theories which involve law-like statements, life sciences like biology and the cognitive sciences typically do not (Bechtel, 2005a, p. 1). ${ }^{21}$ The latter usually appeal to explanations in terms of mechanisms. Mechanistic explanations concern themselves with a system's parts (its constitution/constituents) and their functions. Thus a mechanistic explanation of consciousness would shed light on the constitutive relations between a conscious state and the neural state it correlates with. Mechanistic explanations have been very successful in biology. Since we consider consciousness to be a biological phenomenon, it is fitting that the type of explanation which works best in biology also be applied to consciousness.

Bechtel defines a mechanism as "a system whose behavior produces a phenomenon in virtue of organized component parts performing coordinated component operations" (Bechtel, forthcoming, p. 3). In a typical mechanistic explanation, one attempts to explain a phenomenon (a behaviour of the system) by decomposing the system into component parts and component operations/functions in order to specify how these parts work together to produce the target phenomenon. The operations of a system are usually described from a functional perspective. But a complete mechanistic explanation connects these operations to the parts of a system described from a structural perspective. It tells us that these particular parts function in these specific ways (Bechtel, 2005a, p. 1). 
The main methodological challenge in developing a mechanistic explanation is that mechanisms don't straightforwardly reveal their parts or the functioning of these parts. Experiments are needed for discovering a system's structural and functional properties. But, as illustrated by the following two examples, the structural properties and the functional properties of a system are often discovered by different methods of investigation.

The methods adopted by certain researchers may provide evidence for a system's functional properties while contributing nothing to discovering its structural properties. We see this when we consider that, generally speaking, from a historical point of view, cognitive scientists have been trying to decompose cognition from a functional perspective without having access to methods for decomposing the brain in a pertinent way (from a structural perspective) (Bechtel, 2005a, p. 1). For example, Koch reports the findings of Posner who investigated functions of attention and visual detection of light flashes by using reaction time measurements (Koch, 2004, p. 157). Posner compared how long it took participants to respond to a flash of light somewhere in the visual field in three different conditions. In one condition participants received a cue (an arrow pointing to where the flash would appear). In the other two they either received no cue, or a misleading cue pointing in the direction opposite to where the light later appeared. Reaction times were the fastest in properly cued trials (faster than uncued trials and much faster than misleadingly cued trials). From this Posner concluded that attention speeds up the processing involved in the detection of the light flashes by approximately 30 to $50 \mathrm{~ms}$. But, notice that nothing in this experiment indicates what type of structural 
properties are relevant to the functions of attention and feature detection. This experiment illustrates that experimental methods which shed light on cognitive functions do not necessarily also contribute to the understanding of the mechanisms underlying theses functions from a structural point of view.

Conversely, the methods adopted by certain researchers may provide evidence for a system's structural properties while contributing nothing to discovering its functional properties. This was the case for neuroscientists who developed tools for mapping different areas of the brain from a structural point of view without the benefit of tools for connecting these structurally differentiated brain areas to cognitive functions. For example, taking a structural perspective based on physiological criteria, Brodmann (1912) delineated different brain areas by using staining techniques. He did this in the hopes that the "parts" he identified would eventually be coupled to cognitive functions (the functional perspective) (Bechtel, 2005b, p. 5). But, Brodmann's techniques for mapping the brain provided no clues as to how any of the structural parts he identified could relate to cognitive functioning. ${ }^{22}$

Behind the difficulty of identifying the operations of a mechanism's parts lies the necessity of developing concepts appropriate to characterizing their operations. According to Bechtel, until a suitable conceptual framework which describes the types of functions relevant to a particular phenomenon is developed, researchers habitually investigate levels of organization which are either too high or too low (Bechtel, 2005a, p. 2). (The correct level of organization at which to aim one's analysis is the one which leads to successful theorizing.) 
Researchers aim too high when they conceptualize operations internal to the system by drawing analogies to the behaviours of the overall system. This is exemplified in the approaches of those who see the mind as a symbol processor. Performing operations on symbols is something that we, as cognitive systems, do. Calculating the correct amount to tip a server is an example of such an operation we perform on symbols. By invoking system-level behaviour (symbol manipulation) to explain a behaviour of the system (cognition understood in terms of symbol manipulation), cognitive research is, in a sense, assuming what it is trying to explain. Approaches which invoke representations to explain cognition similarly also aim too high (Bechtel, 2005b, p. 2). But, in addition to this problem of circularity, there is the problem of using things in an explanation (like "representations") that are almost as complicated as the phenomenon we're trying to explain (Brook, forthcoming, p. 1). In a good explanation, complex phenomena are explained in terms of more basic phenomena. Or else, we risk merely redescribing the target phenomenon, instead of actually explaining it.

Researchers aim too low when investigating very low levels of organization that seem unconnected to the system's (externally accessible) behaviour. Taking an example from outside of cognitive science, it will do no good to attempt an explanation of how a windmill converts wind power into other forms of energy (a system-level behaviour) by appealing to analyses of the chemical constitution of its blades or gears. To explain the windmill's behaviour we need to look higher than the level of chemical constitution. We need to look at the level of it parts and their arrangements. After another example, this 
one drawn from the history of biochemistry, we'll get to some examples of approaches which aim too high/too low in the context of the NCC search.

Bechtel offers a historical example from biochemistry which illustrates the difficulty of conceptualizing the functions of a mechanism's components at an explanatory useful level of organization. The $19^{\text {th }}$ century saw many efforts at explaining physiological processes such as fermentation and respiration. The chemical revolution of the late $18^{\text {th }}$ century isolated the main elemental constituents of living organisms (carbon, hydrogen, oxygen, and nitrogen). Many attempted to build upon these fruits of the revolution and sought to explain physiological processes on the basis of modifications in elemental composition. However, research at the level of elemental composition didn't lead to any successful explanations of biological processes. (There was no way to make the connection between this low level of organization and the system's behaviour (fermentation).)

Towards the end of the $19^{\text {th }}$ century it was discovered that chemical substances are not merely groups of atoms but structured groups of atoms. As a consequence, chemical structure, and not only elemental composition became important to explanations of physiological processes. It was discovered that substances like glucose could be broken down into component substructures. Thinking about chemical reactions in terms of interactions between substructures raised the question of whether certain substructures were intermediaries in the process of fermentation. Bechtel points out that what was significant about attempts at explanations at the level of these chemical substructures was that researchers described the possible component operations (the functions of the 
chemical substructures) in the same terms which were used to describe the behaviour of the whole system (Bechtel, 2005a, p. 3). For example, attempts at explaining alcoholic fermentation appealed to fermentations as intermediate processes, thus invoking operations at the same level as the overall system's behaviour. Because these attempts didn't explain fermentation in terms of something more basic, no useful explanations were constructed at this level of organization.

Then there was a breakthrough: organic chemists discovered that organic substances consist of groups of molecules which are bound to carbon rings. In a chemical reaction, whole groups of molecules are added to or deleted from these rings. Conceptualizing chemical reactions at the level of groups of molecules lead to fruitful theorizing about the intermediate steps (the component functions) in many physiological processes. And thus the foundation of biochemistry was laid. It turned out that the proper level of organization at which to conceptualize the mechanism's functions was higher than the level of elemental constitution. But, functions had to be conceptualized at a level of organization which allowed for explanations of the phenomenon in terms more basic than those used to describe the behaviour of the whole system.

It is Bechtel's belief that cognitive scientists trying to explain cognitive functioning find themselves in a position similar to that of the chemists before the framework of groups of molecules (Bechtel, 2005a, p. 1). They investigate levels of organization which are either too low (and don't connect with the target behaviour) or too high (motivating explanation at (whole) system-level organization which connects to the target behaviour but does not lead to useful theorizing). 
We also find similar cases in the context of the NCC search. Here are two examples of aiming one's analysis too low and too high when trying to explain conscious functions or other cognitive functions (a cognitive system's behaviour) by searching for their neural correlates.

Certain mechanisms, such as the action potentials of neurons, have been identified at low levels of neural organization. If these mechanisms are the neural correlates of conscious functions, could they lead to explanations of conscious functions? Although we have a fairly good understanding of the mechanism of action potentials we don't know how they could connect to conscious functioning (the system's behaviour). For instance, knowing that a cell is activated in response to a certain type of stimulus doesn't tell us what role the cell might play in the processing that results in consciousness of the stimulus (Bechtel, forthcoming, p. 14). What makes mechanisms of action potentials too low when trying to explain consciousness or other cognitive functions is that we don't know how to aggregate neurons and neuronal assemblies into mechanisms that can explain the target phenomenon. This is why neurons/neuronal assemblies and their activities seem unconnected to consciousness. (This seeming lack of a connection is an example of the "explanatory gap" which we discuss next chapter.)

Here is an example of an approach to functions which appears to aim at too high a level of neural organization. Functional magnetic resonance imaging (fMRI) is an approach which attempts to link brain activity to conscious functioning. ${ }^{23}$ Even if many brain areas were identified as being part of a network which is activated during a particular conscious act, it tells us nothing about what each area does (Bechtel, 2005a, 
p. 5). Finding this sort of neural correlate doesn't tell us what the component parts and component functions are or how they relate to other layers of mechanism. Although the correlated function connects with the system's behaviour, it seems unconnected to lower levels of neural organization (lower layers of mechanism). (In other words, when we see the brain activation measured by fMRI, we don't see that the result must be consciousness - here again is an example of the "explanatory gap.")

Is searching too high or too low also the plight of cognitive scientists engaged in the search for the NCCs of unified consciousness? This question and the methodological problems and conceptual issues presented by the study of the NCCs of unified consciousness are the focus of the remainder of this work.

We shall see that, as the chemists looking at elemental composition, researchers taking "local" approaches to NCCs find themselves searching at levels of neural organization which appear too low when attempting to account for the unity of consciousness. In particular, these approaches appear inadequate to accounting for the binding implied by unified forms of consciousness. We consider "local" approaches in relation to unified consciousness in Chapter 3. In Chapter 4, we consider more "global" approaches to unified consciousness. Most work specifically focusing on the neural correlates of unified forms of consciousness uses measures of "neural synchrony." While those adopting "global" approaches believe that synchrony studies hold the promise of accounting for the binding implied by unified forms of consciousness, we aim to show that is not the case. In light of the framework set out in the present chapter, "global" 
approaches aim at levels of organization which are too high. (For instance, they don't allow for the localization of the parts of the mechanisms of unity.) 


\section{On the Search for the Neural Correlates of Unified Consciousness}

\section{Chapter 3}

\section{1 - Introduction}

A general methodological problem in NCC research is determining the correct level of analysis at which to search for NCCs. Researchers adopting "local" and "global" approaches differ as to the level of neural organization they deem relevant to the NCC search. In this chapter we see how local approaches fare at meeting the particular challenges posed by the unified character of consciousness (such as the binding problem(s)). Local approaches need to handle such problems if they are to lead to the discovery of the neural correlates of unified consciousness. (We consider global approaches to the neural correlates of unified consciousness in the next chapter.) First, however, we discuss what is meant by the unity of consciousness.

\section{2 - The notion of unity: two forms of unity and Gestalt relationships}

There are many different types of unified consciousness. ${ }^{24}$ Here we focus on two forms: unified consciousness of individual objects and unified consciousness of multiple objects. In both unified consciousness of individual objects and unified consciousness of many objects, all "parts" (features or objects) are taken up into a whole. The main difference between these two types of unity is a difference of degree. Because they differ as to how many features/objects are united, these forms reflect different levels of unified conscious experience. They are both types of consciousness of the world as opposed to consciousness of one's conscious states (reflexive or state consciousness). 
Note that it is not our aim to set out the necessary or sufficient conditions of unity. ${ }^{25}$ Our goal here is to illustrate what the unity of consciousness is by drawing attention to unity as it presents itself in common conscious experience.

To understand the notion of unity, it is useful to think in terms of part/whole relations. We can get at the notion of unity by way of a distinction between a sum of parts and a complex of parts. A molecule is a good example of a complex (Kriegel, 2006, p. 150). A molecule (the whole) is a complex of atoms (the parts). The reason a molecule is not merely a sum of atoms is that, for it to be the type of molecule it is, the atoms must be interconnected in a particular way. Two hydrogen atoms and an oxygen atom only compose a water molecule if they are interconnected in a specific way. If the three atoms were simply lumped together without sharing these connections, we would have a mere sum of atoms (a sum of parts) and not a water molecule (a unified complex comprised of interconnected parts). The particular way that atoms are interconnected makes a molecule into the sort of molecule it is. Similarly, the particular way the distinguishable "parts" of my conscious state are experienced as interconnected makes it the sort of experience it is. As the molecule analogy brings out, to say that there is unity in consciousness is to say that a certain relation (or set of relations) holds between conscious items. To illustrate some unity relations, let's look at our first type of unity: object unity. This type of unity characterizes our visual experience of everyday objects. Objecthood requires unity. Without it we would not experience any objects - only aggregates of features. I see the apple before me, not as a confused bundle of discrete and unconnected features, but as an organized whole comprised of different features (shape, colour, movement, etc.) 
which can be thought of as parts. The different parts are related to one another in such a way that they are experienced as belonging to a whole (the apple). For instance, the redness of the apple bears a very close relation to its shape. They occupy the same space. Also, if I knock the apple over, its movement is experienced as belonging to the whole, not exclusively to its colour rather than to its shape. This set of unity relations between the different features yields an experience of the apple as a whole, rather than simply as a loose collection of unrelated features/parts.

To help get at the unity relation and at the same time serve to introduce our second form of unity, unified consciousness of multiple objects, let's say you pick up the apple to examine it. Your experience of looking at the apple held in your hand won't be of a series of discrete "smaller" experiences of the apple's colour, its shape, its texture, its weight, feelings of hunger or thirst, the sound of geese flying overhead, etc. At one instant, many of these visual, tactile, and aural elements/objects combine, or are united into the same complex conscious experience. This unity between multiple and multimodal objects is characteristic of common conscious experience. In consciousness, unity is ubiquitous. Even if we restrict ourselves to vision, in a single instant, when I look at the calculator on the desk, I also see a pen, some books in a pile, and papers. ${ }^{26}$ Although objects surrounding the calculator appear less defined, all are experienced as part of the same scene.

These two forms of unity, object unity and global unity of multiple objects, were already recognized by Kant (Brook \& Raymont, 2006, p. 5). Kant believed that the unity of objects resulted from a form of synthesis carried out by the mind. He distinguished this 
form of synthesis from another form which is responsible for combining the various unified objects into our rich unified conscious experience (Brook, 1994, pp. 123-124). This second form of synthesis is what integrates the various conscious individual objects into larger states of globally unified multiple objects. It is hard to discount the importance of this type of unity for cognition. Without it, we couldn't relate or compare the different items we experience to one another. This ability to compare different elements is fundamental to our type of cognition (Brook \& Raymont, 2006, p. 5).

"Gestalt relationships" hold between the global structure of a unified conscious experience and the parts that are distinguished within it. They can be found in part/whole relations at both the level of many unified objects, and at the level of individual unified objects.

Let's begin with Gestalt relationships at the level of globally unified experiences of many objects. When individual objects (the parts) are unified into a globally unified experience of many objects (the whole), Gestalt relationships can obtain between the whole and its parts. Sometimes, when having unified consciousness of multiple objects, the particular way an individual object appears is a function of the whole display (Brook \& Raymont, 2006, p. 3). In other words, changing the overall display can affect the experience of the individual objects which are parts within it. For example, changing the background sometimes affects the way objects in the foreground appear. ${ }^{27}$ If it were surrounded by other similarly-oriented and identical-looking calculators, the calculator in the foreground of my visual experience would lose some of its saliency, and tend to blend in with the background. The fact that some objects or elements of a scene appear to be in 
front of others, which are experienced as in the background, is an example of a Gestalt relationship. And now for an example of a Gestalt relationship at the (lower) level of individual unified objects.

Part/whole Gestalt relationships can also arise between a section of a scene (like an individual object) and a smaller part it contains (like one of the object's features). In a visual scene comprised of many different objects, an object can function as a whole within the scene. Take a Kanizsa triangle. This figure is composed of three circles with triangles cut out of them. Each circle looks like Pac-Man from the popular 1980s videogame (Figure 1). Due to the particular way the "pacmen" are arranged, an illusory triangle emerges (Figure 2) ${ }^{28}$ Because the illusory triangle (the whole) depends on the particular arrangement of its parts (the "pacmen"), if the "pacmen" are rotated so that they are no longer facing each other, the Gestalt disappears (Tallon-Baudry et al., 1996, p. 4241). But to experience the Gestalt is to experience more than these parts - we see the triangle. 


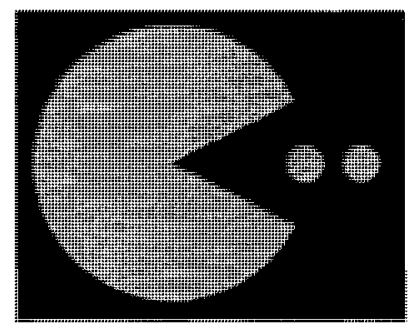

Figure 1 - Pac-Man

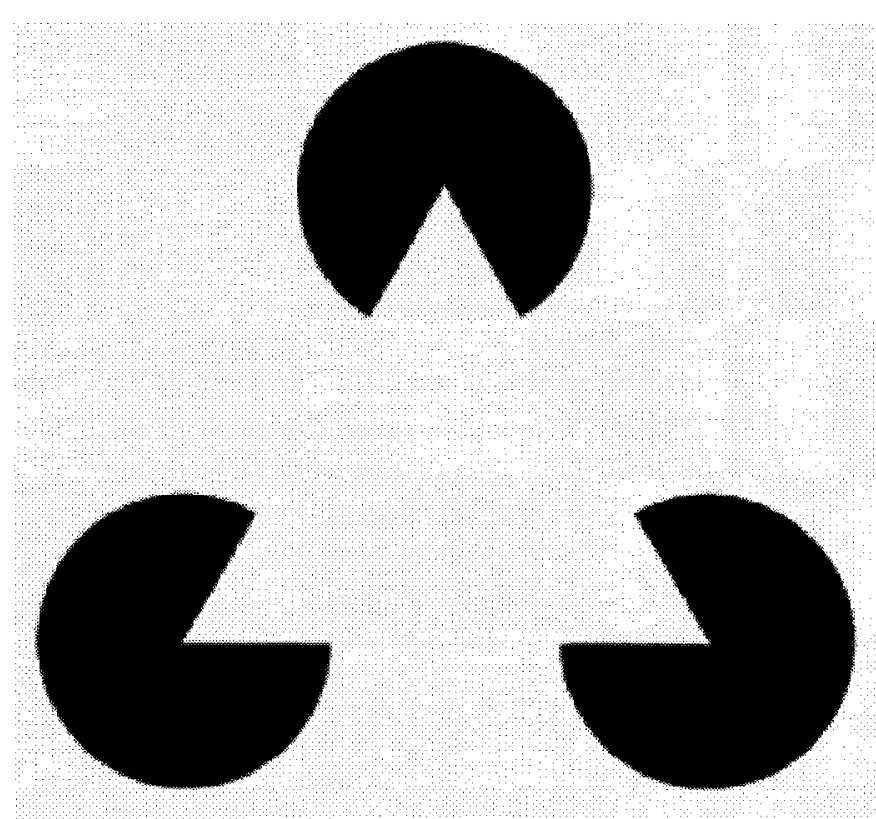

Figure 2 - A Kanisza Triangle

Such illusory Gestalts are useful to the search for the neural correlates of unified consciousness. Stimuli involving illusory objects which arise from part/whole Gestalt relationships play an important role in the experimental paradigm we propose for isolating two different functions of unity (in Chapter 4). These different functions correspond to the two types of unity introduced in the present chapter. The "individual object unity" function corresponds to the unified consciousness of individual objects. The “multiple object unity" function corresponds to the globally unified consciousness of many objects.

The centrality of unity in consciousness is brought out by cases in which neurological damage causes it to become fragmented. For example, patients who have Balint's syndrome (as a result of bilateral damage to the parietal lobes) can experience the world in a strikingly disunified way. Some of these patients have simultanagnosia and 
are unable to perceive more than one object at a time. Erroneous conjunctions of features are also common in these cases. For instance if a blue $X$ and red $O$ are presented to the patient, she may report seeing a red $X$ or a blue $O$ (Revonsuo, 2006, p. 214). Because the experiences of these patients may strike us as bizarre, they highlight the fact that unity is a central characteristic of common experience.

Although we have serious doubts on the matter, we will not get into the question of whether or not people sometimes have experiences completely devoid of unity or even whether it is possible to coherently imagine such a situation. The fact that, in common conscious experience, unity is ubiquitous, is enough to justify the requirement that theories about consciousness must account for or, at the very least, be compatible with unity in their explanations. But, as will soon become apparent, the unity characteristic of consciousness poses particular problems for the search for the neural correlates of unified consciousness.

3.3 - Phenomenal unity implies the integration of distributed information on the neurological level

The forms of unity just discussed are forms of unity as they are experienced. They are types of phenomenal unity. The search for possible mechanisms that could realize the unity of consciousness naturally leads to discussions of the binding problem, a popular conundrum in cognitive science. Even the perception of a simple unified visual percept such as a square involves the coming together of different features which are processed by different areas of the brain (Humphreys, 2003, p. 115). Yet, somehow, the square 
appears to us as a unified object and not just a haphazard combination of its different features.

Because conscious experience involves many different levels of binding, the binding problem is really a collection of related problems. For example, the two types of unity we've introduced reflect different levels of binding. Unified consciousness of multimodal objects involves binding at a scale much larger than that involved in the unimodal visual experience of an individual unified object. On the assumption that the brain somehow encodes perceptual information, there must be a neural mechanism responsible for the integration of this spatially distributed information. From the neurological point of view, the binding which results in our typically rich and multimodal conscious experience necessitates the integration of information processed by many specialized areas spread out across the brain.

Different levels of binding imply the integration of perceptual information at different scales of neural organization. There is integration on a large scale when neural groups located in different parts of the brain are united into a network. There is small scale integration when the information within an area or from neighbouring cortical areas is united. For instance, areas specialized in treating information related to the same modality are often found close together. The integration of information within the visual system can be considered small scale information integration. Questions about which scale of neurological organization one should study when looking for the neural correlates of unified consciousness reflect a methodological problem for NCC research: the problem of choosing the right level of analysis. 


\section{4 - Methodological problem: What is the right level of analysis?}

In Chapter 2, we saw that when developing a mechanistic explanation, a fundamental challenge is finding the proper level of analysis (or level of description) of the system's organization which leads to the construction of better theories. In NCC research this problem becomes how to identify and describe the appropriate level of neural organization. The proper level of neural organization at which NCCs should be sought is the main point of contention between researchers adopting local approaches and those taking more global ones.

NCCs can be individuated in various ways and at various levels of description. We can rank neuroscientists' approaches to individuating NCCs on a scale ranging from local approaches (at the neuronal or even subneuronal level) to more global approaches involving more widespread activity in the nervous system. "Local" and "global" refer to levels of neural organization (the neurological basis of the experience). They aren't meant as descriptions of experience itself.

In local approaches, the units of analysis are usually very fine-grained while global approaches involve coarser-grained units of analysis. In contrast to local properties like firing rate and subcellular processes, which are measured at the level of single cells or a few cells, more global properties like synchronous spiking activity (or "neural synchrony") are measured at the level of larger distributed groups of neurons. But, the issue of levels of analysis is not only about grain size - it is also about having the right vocabulary for describing the functions of a mechanism. (Recall that before chemists adopted the vocabulary describing chemical reactions at the level of groups of molecules, 
biological functions like fermentation were not construed at an explanatorily useful level of organization.)

At the local end of the spectrum, Koch thinks that multiple forms of neural activity and anatomical features at the single-cell level may be important to the NCCs. The location of the neurons may also be a key factor. He even suggests that the NCCs could be intimately linked to subcellular processes such as the concentration of calcium ions within neurons (Koch, 2004, p. 17). Taking an extremely local approach, Hameroff applies quantum theory to microtubules which are subcellular structures. More than simply claiming that microtubules correlate with consciousness, he hypothesizes that consciousness is to be found in microtubules, because a series of quantum mechanical events in the microtubules creates consciousness (Hameroff \& Penrose, p. 457).

Others are convinced that a more global analysis is in order. For example, rather than look at the local properties of neurons, Tononi and Edelman focus on the interactions between large distributed groups of neurons. They believe that explanations of fundamental properties of conscious experience (such as unity) should be sought by connecting these conscious properties to more global properties of neural processes (like complex patterns of activation) (Tononi \& Edelman, 1998, p. 1850). Some take an even more global approach, insisting that we should look for more than the neural correlates of consciousness, and include aspects of the subject's environment. Noë and Thompson believe that the correlates of consciousness extend beyond the borders of the skull and skin (Noë \& Thompson, 2004). 
At what level of neural organization should we look for the NCCs of unified consciousness? According to Revonsuo, "the deepest theoretical problem in neuroscience" is the methodological problem of discovering the correct level of analysis (Revonsuo, 1999, p. 181). The correct level of neural organization at which neural correlates of unified consciousness should be analyzed will be whatever level (or levels) of neural organization turns out to be the one which leads to fruitful theory construction.

\section{5 - One source of the correct level of analysis problem: Neural coding}

One source of the methodological problem of how to determine the proper level of analysis finds itself in the lack of consensus, among neuroscientists, about how the brain codes information. Accepting a theory of coding will constrain the relevant levels of analysis. (Likewise, different levels of analysis can call for different coding strategies.)

Two main types of coding strategies are firing rate coding and temporal coding. Those favouring local approaches tend to assume only the former, while those in the globalist camp accept temporal coding as an additional coding dimension.

Choosing between rate coding and temporal coding depends, in part, on the level of phenomenal unity of the target phenomenon (in virtue of the scale of neurological information integration thought to underlie this unity). The local perspective focuses on conscious phenomena involving very low levels of phenomenal unity. Coding by spiking discharge seems to suggest a plausible mechanism for the corresponding low level of information integration. (For example, increased firing rate between interconnected neurons is believed to unite the cells coding for a particular feature of a percept. But this 
coding strategy, as we'll see, runs into problems when many objects are involved.) The global perspective studies conscious phenomena involving higher levels of unity. Temporal coding strategies like "neural synchrony," favoured by global approaches, appear more germane to dealing with the corresponding higher levels of information integration from the neurological perspective. Thus, because of the scale of information integration it implies from the neurological point of view, the level of phenomenal unity of the conscious phenomenon in the explanandum largely dictates the level of analysis (on the local to global scale). The more the phenomenon to be explained exhibits unity, the more global the approach.

Global approaches invoking synchrony to account for the unity of consciousness are considered in Chapter 4. Local approaches, firing rate coding, and the unity of consciousness are the main focus of the remainder of the present chapter. However, before seeing how local approaches invoking the rate code could deal with the information integration underlying the unity of consciousness, we take a step back to look at coding more generally. This will help us get an idea of how neurons could encode information and it will also lead to a discussion of the "explanatory gap," a problem concerning consciousness in general.

\section{6 - $\underline{\text { How neurons could encode information }}$}

In both the firing rate and temporal views of coding, neurons are assumed to be the fundamental units of perceptual/cognitive processing which encode the relevant information. The view that neurons are the fundamental components of the nervous 
system and that the brain's functioning can be understood by their actions and interactions is known as "the neuron doctrine" (Kaczmarek, 2002, p. 325). This doctrine is widely accepted by contemporary neuroscientists. Although neurons can influence other neurons in various chemical and mechanical ways, "synaptic transmission" is the type of neuronal interaction which is usually identified with the doctrine (Kaczmarek, 2002, p. 326). A synapse is the area where one neuron comes into contact with another neuron. Neurons are traditionally described as having three functional parts: the dendrites, the cell body, and the axon. After traveling the long of the axon and reaching the synapse, an action potential causes the release of chemical neurotransmitters which in turn excite or inhibit the dendrites of a connected neuron. (Action potentials are electrical impulses or spikes.) Exciting the connected neuron either triggers an action potential in it or increases the chances of triggering one, while inhibiting the post-synaptic neuron diminishes the chances it will fire.

Figure 3 - Neuron

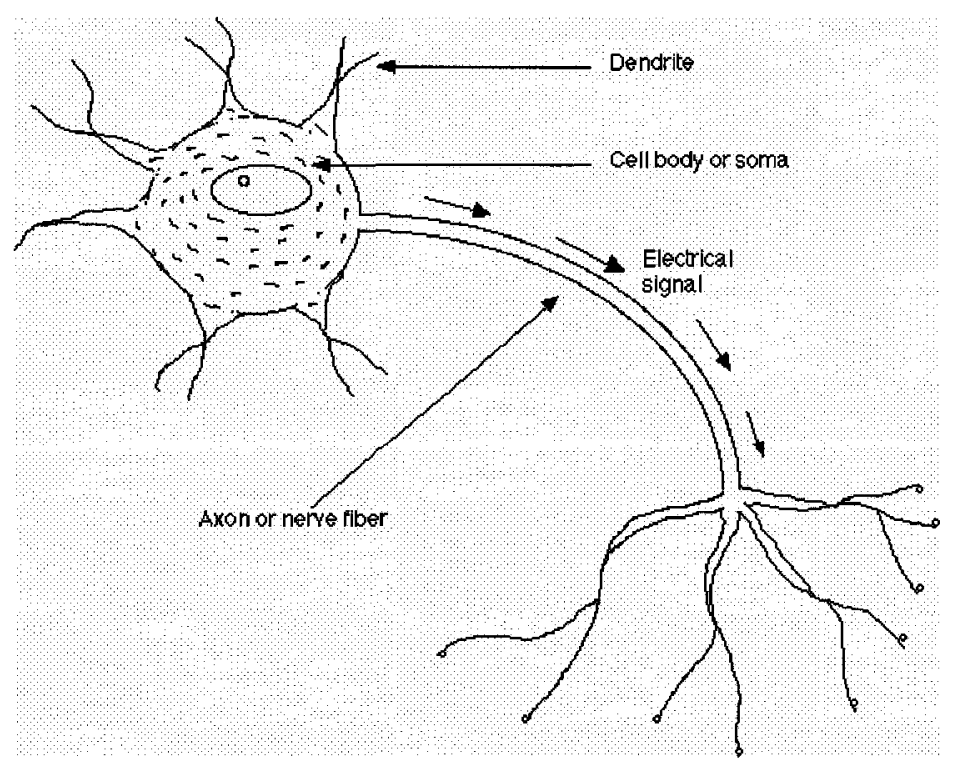


Neuroscientists assume that neurons and their spiking activities (somehow) encode perceptual information. But, neurons also need to be able to encode rules. Much of our cognition is rule-governed. (Think of the rules governing language production, for instance.) Even binding seems to work according to rules such as "if two properties occupy the same space, then they belong to the same object."

How could neurons implement rules? Using the mathematical function of addition, we present an example of how neurons and their spiking activities could implement a rule. This neuronal circuit is built using "McCulloch-Pitts neurons" whose functioning is an abstraction of the computational functioning of biological neurons (Anderson, 2002, p. 1043).

In a 1943 article, McCulloch \& Pitts, now considered pioneers of neural network modeling, demonstrated that the basic logical functions ("And", "Or", "Not") could be computed by circuits made up of abstracted neurons (Levine, 2000, p. 12). The networks they proposed are comprised of excitatory and inhibitory neurons. A neuron fires if the sum of incoming excitatory and inhibitory spikes reaches the neuron's threshold. An assumption of the model is that computations proceed in discrete time intervals. Another assumption is that, at each interval, any neuron finds itself either in the firing ("on" or "1") or not firing ("off" or "0") state. Thus, they behave in an "all or none" manner.

This neuron model is generally considered an oversimplification of a biological neuron's real functioning. In particular, its "all or none" nature conflicts with the behaviour of real neurons which often appear to function like analogue devices, using continuous values rather than the discrete ones used by digital devices (Anderson, 2002, 
p. 1045). But, the McCulloch-Pitts neuron model can still serve as a possibility proof for how groups of neurons could be capable of encoding rules.

\section{Table 1 - Binary Numbers}

\begin{tabular}{|c|}
\hline Decimal $=$ Binary \\
\hline $0=0$ \\
$1=1$ \\
$2=10 \leftarrow$ \\
$3=11$ \\
$4=100$ \\
$5=101$ \\
$6=110$
\end{tabular}

Our circuit will compute the addition function by using the binary code. ${ }^{29}$ Notice that to represent the number two, it takes two binary symbols ("bits").

Table 2 - Binary Addition

$$
\begin{aligned}
0+0=0 \\
0+1=1 \\
1+0=1 \\
1+1=10
\end{aligned} \quad \leftarrow \text { Notice that we need a way to carry the extra bit. }
$$

Now, let's represent the binary addition (of two bits) in the form a table with "inputs" and "outputs."

Table 3 - Binary Addition

(Input-Output Possibilities)

\begin{tabular}{|c|l|l|l|}
\hline \multicolumn{2}{|c|}{ INPUTS } & \multicolumn{2}{l|}{ OUTPUTS } \\
\hline A & B & Carry & Sum \\
\hline 0 & 0 & 0 & 0 \\
\hline 0 & 1 & 0 & 1 \\
\hline 1 & 0 & 0 & 1 \\
\hline 1 & 1 & 1 & 0 \\
\hline
\end{tabular}

The carry output is an "And" function. The sum output is an "Exclusive-Or" function.

So, if we can build these logical functions out of "McCulloch-Pitts neurons" we'll have a neuronal circuit which can add two single-bit numbers together. 
Here is our "And" circuit (Figure 4). The circles are neurons. The numbers are the thresholds which must be met for the neurons to fire. The "plus" symbols indicate excitatory connections. ${ }^{30}$

Figure 4 - "And" Circuit

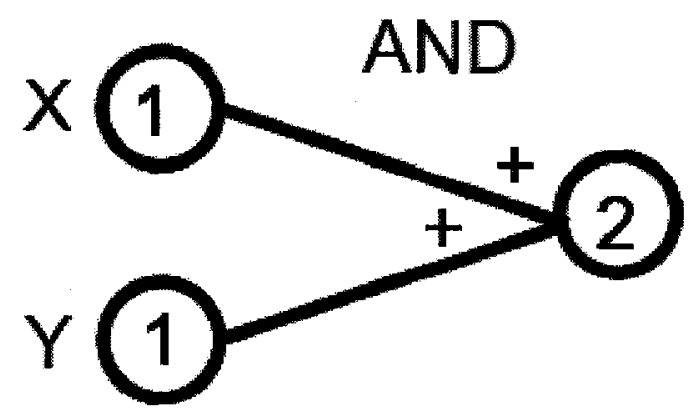

Table 4 - "And" Circuit (Input-Output Possibilities)

\begin{tabular}{|l|l|l|}
\hline $\begin{array}{c}\text { Input } \\
(\mathrm{X})\end{array}$ & $\begin{array}{c}\text { Input } \\
(\mathrm{Y})\end{array}$ & Out \\
\hline 1 & 0 & 0 \\
\hline 0 & 1 & 0 \\
\hline 1 & 1 & 1 \\
\hline 0 & 0 & 0 \\
\hline
\end{tabular}

Table 4 represents all possible combinations of inputs and outputs for our "And" circuit. The reader will observe that it is the truth table for the "And" function.

Figure 5 - "Exclusive-Or" Circuit

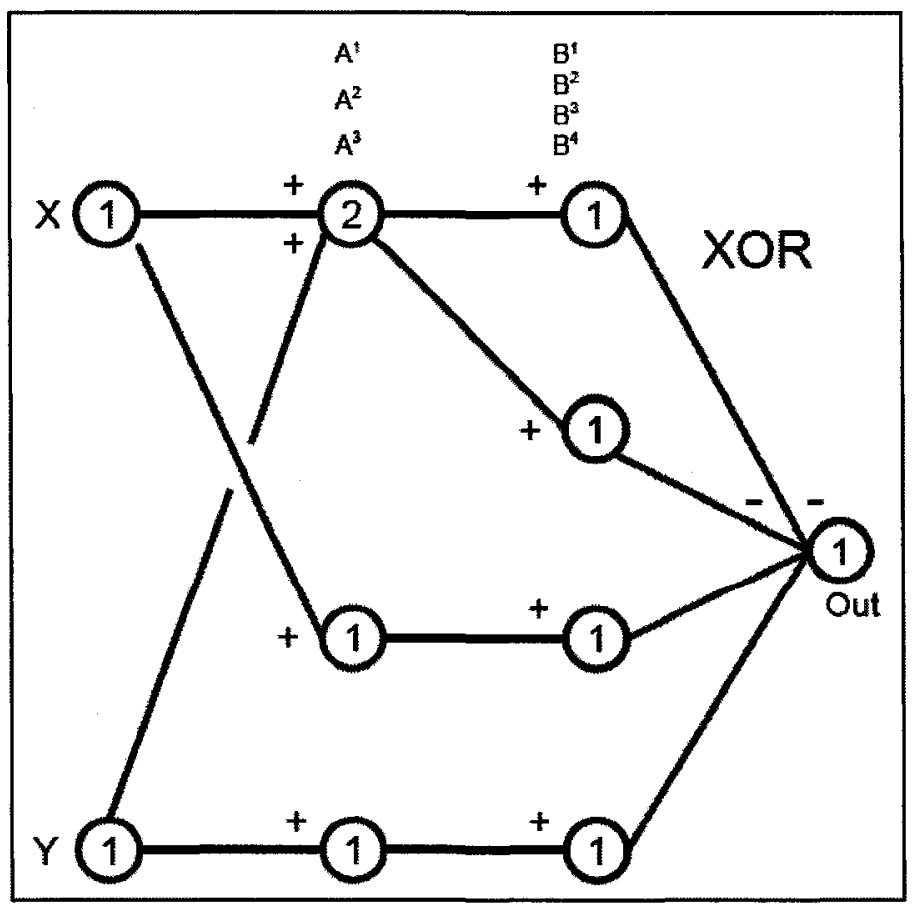

To the left is our "Exclusive-Or" circuit. Minus symbols indicate inhibitory connections. 
Tables 5-7 represent the possible combinations of its inputs and outputs. Computations follow discrete time intervals (left column).

Tables 5-7 "Exclusive-Or" Circuit (Input-Output Possibilities)

\begin{tabular}{|l|l|l|l|l|l|l|l|l|l|l|}
\hline Time & $\mathrm{X}$ & $\mathrm{Y}$ & $\mathrm{A}_{1}$ & $\mathrm{~A}_{2}$ & $\mathrm{~A}_{3}$ & $\mathrm{~B}_{1}$ & $\mathrm{~B}_{2}$ & $\mathrm{~B}_{3}$ & $\mathrm{~B}_{4}$ & Out \\
\hline 1 & 1 & 0 & 0 & 0 & 0 & 0 & 0 & 0 & 0 & 0 \\
\hline 2 & 0 & 0 & 0 & 1 & 0 & 0 & 0 & 0 & 0 & 0 \\
\hline 3 & 0 & 0 & 0 & 0 & 0 & 0 & 0 & 1 & 0 & 0 \\
\hline 4 & 0 & 0 & 0 & 0 & 0 & 0 & 0 & 0 & 0 & 1 \\
\hline
\end{tabular}

\begin{tabular}{|l|l|l|l|l|l|l|l|l|l|l|}
\hline Time & $\mathrm{X}$ & $\mathrm{Y}$ & $\mathrm{A}_{1}$ & $\mathrm{~A}_{2}$ & $\mathrm{~A}_{3}$ & $\mathrm{~B}_{1}$ & $\mathrm{~B}_{2}$ & $\mathrm{~B}_{3}$ & $\mathrm{~B}_{4}$ & Out \\
\hline 1 & 0 & 1 & 0 & 0 & 0 & 0 & 0 & 0 & 0 & 0 \\
\hline 2 & 0 & 0 & 0 & 0 & 1 & 0 & 0 & 0 & 0 & 0 \\
\hline 3 & 0 & 0 & 0 & 0 & 0 & 0 & 0 & 0 & 1 & 0 \\
\hline 4 & 0 & 0 & 0 & 0 & 0 & 0 & 0 & 0 & 0 & 1 \\
\hline
\end{tabular}

\begin{tabular}{|l|l|l|l|l|l|l|l|l|l|l|}
\hline Time & $\mathrm{X}$ & $\mathrm{Y}$ & $\mathrm{A}_{1}$ & $\mathrm{~A}_{2}$ & $\mathrm{~A}_{3}$ & $\mathrm{~B}_{1}$ & $\mathrm{~B}_{2}$ & $\mathrm{~B}_{3}$ & $\mathrm{~B}_{4}$ & Out \\
\hline 1 & 1 & 1 & 0 & 0 & 0 & 0 & 0 & 0 & 0 & 0 \\
\hline 2 & 0 & 0 & 1 & 1 & 1 & 0 & 0 & 0 & 0 & 0 \\
\hline 3 & 0 & 0 & 0 & 0 & 0 & 1 & 1 & 1 & 1 & 0 \\
\hline 4 & 0 & 0 & 0 & 0 & 0 & 0 & 0 & 0 & 0 & 0 \\
\hline
\end{tabular}

Now, let's combine both the "And" circuit and the "Exclusive-Or" circuit into a two-bit adder circuit (Figure 6). ${ }^{31}$ This two-bit adder circuit can perform the computations in Table 3. 
Figure 6 - Two-Bit Neuronal Adder Circuit

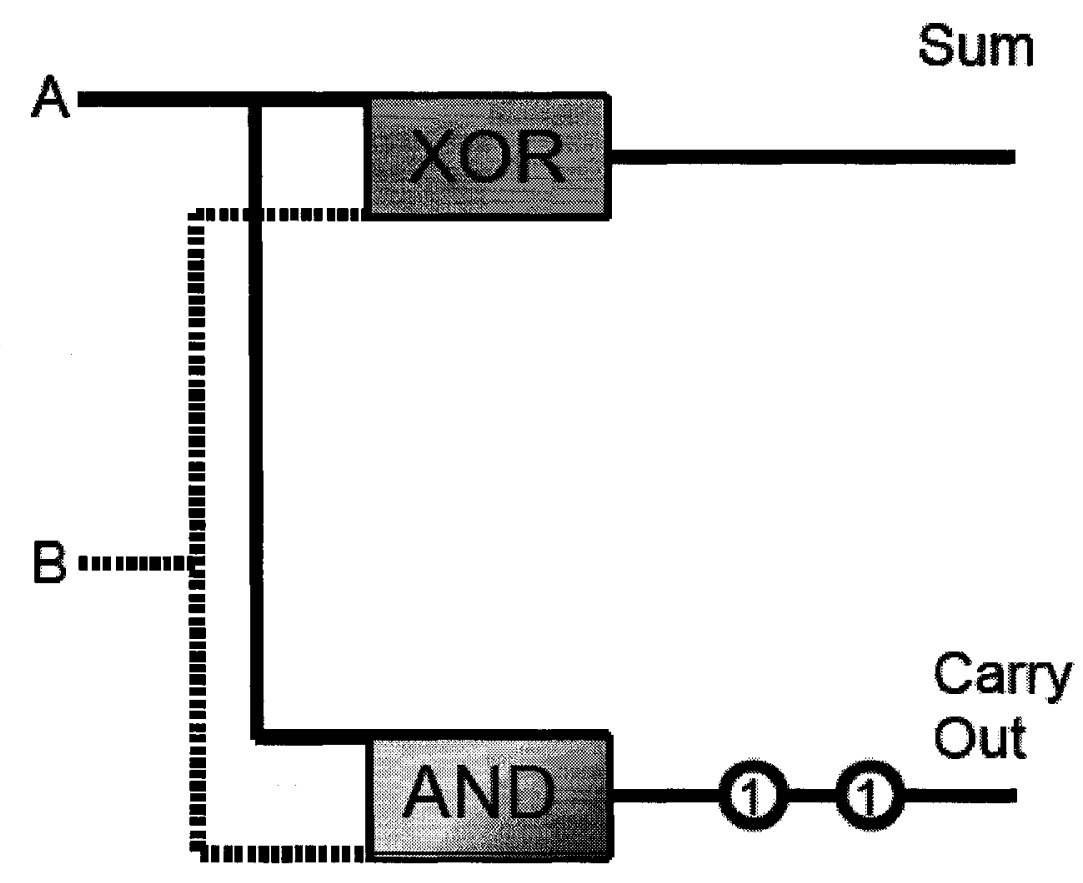

If we wanted to add more than two bits together, we could do so by putting many two-bit adders together in parallel. Because we can build all logical functions from the three basic logical functions ("And", "Or", "Not”), we could also build circuits to perform subtraction, multiplication, and division.

This neuronal adder circuit is an example of a simple information processing mechanism because it specifies how the parts (neurons in a particular arrangement) interact (by producing spikes) to accomplish a function (addition).

If the neuron doctrine is true, in addition to being able to encode rules, neurons must also be able to retain information to allow for functions of memory, for example. Again, this doctrine assumes that information is coded by the action potentials of 
neurons. But how could information in the form of spikes be stored over time $?^{32}$ This puzzling question invites others, such as how does a neuron's spiking activity relate to our rich conscious experience?

\section{7 - The "explanatory gap" (or the "hard problem")}

Knowing that a neuron responds to a particular stimulus by increasing its number of action potentials does not tell us how actions potentials (or any other neural mechanism) could result in consciousness.

The lack of agreement on a theory of coding reflects the more general problem of understanding the relation between neural/physical activity and consciousness. This problem is an example of what Levine called an "explanatory gap" (Levine, 1983, p. 354), and it has been described by Chalmers as the "hard problem" of consciousness (Chalmers, 2002, p. 248). In a nutshell, the "hard problem" is finding answers to these questions: (a) Why do we feel anything at all (why is there such a thing as phenomenal consciousness)? (b) How is it that we feel (how could physical processes give rise to phenomenal consciousness)? A related problem is answering the question (c) Why is it that this particular neural activity feels like this rather than like that? A fourth related problem is the question of how homogeneous neural activity could possibly code and account for the great variety and richness of conscious experiences. These problems, at least at present, seem intractable.

In terms of mechanistic explanation, the general explanatory gap becomes a question of missing layers of mechanism. Ultimately, if it turns out that consciousness 
can be explained in terms of mechanisms, for a given type of unified consciousness, there would be an upper-most layer. If we can show that, when the parts in this layer function in a certain way, there must be unified consciousness, then we will have discovered the mechanism of this type of unified consciousness. Of course, a complete mechanistic explanation also necessitates that the functioning of each hierarchically organized layer be explained by the functioning of the layer directly beneath it. So, for example, if we discovered that the mechanism of action potentials is part of the overall mechanism which realizes consciousness, then we would know how it explains the mechanism at the level directly above it. But there is a seemingly large explanatory gap between low-level mechanisms (like action potentials) and consciousness.

Let's put these seemingly baffling problems aside and assume, along with the majority of neuroscientists, that neurons and their activities are somehow involved in the encoding of conscious information. We now move away from problems with consciousness (and coding) in general to return to problems raised specifically by its unified nature.

\section{8 - Local approaches, firing rate coding, and unity}

If one considers coding by firing rate as the only coding strategy available to the brain, then one can look for the NCCs by aiming one's analysis at levels of neural organization starting from single neurons and up to groups of neurons. (The relevant units of analysis are single cells, or a few cells, and larger populations of cells.) Thus, to limit oneself to rate coding implies a local approach. 
We've mentioned that neurons are generally believed to process information by generating action potentials (electrical impulses or spikes). There is variation in the timing and number of these spikes (Földiák, 2002, p. 340). In coding by firing rate, the average number of spikes, recorded over repeated stimulations, is what is considered relevant. ${ }^{33}$ It is assumed that spiking activity is an index of cognitive or conscious activity. For example, if a certain neuron increases its spiking frequency only when the subject has the experience of seeing a moving bar, based on this correlation, the increased neural activity will be thought to somehow participate in the encoding for the conscious act of seeing moving bars.

This type of coding does not come for cheap in terms of the number of neurons needed. But, since on the firing rate view many neurons code for roughly the same features, this type of coding is robust to damage (damage to some cells can be compensated for by activity in the remaining cells) (Koch, 2004, p. 37).

Individual cells that respond selectively to a particular stimulus (say the sight of a cat or your grandmother) have been found in humans. In the 1950s, Penfield electrically stimulated the brains of patients preparing for surgery related to severe epilepsy by placing an electrode on or in the cortex. He discovered that these stimulations sometimes triggered vivid conscious experiences in patients such as visions, hearing music or having feelings from their past (Penfield \& Perot, 1963, p. 596). Discoveries like these led some to speculate that the brain may code information by having highly specialized "grandmother cells," each coding for different percepts. ${ }^{34}$ However, this coding strategy is generally dismissed as not allowing for enough representational capacity. There simply 
aren't enough cells to code for every distinct possible conscious experience we have in a lifetime (Revonsuo, 2006, p. 270). ${ }^{35}$ In population coding many individual cells act as a group. Population (or assembly) coding allows for much more representational capacity since one individual cell can, at different times, participate in many different assemblies. Because it allows for combinatorial coding, most neuroscientists believe that perceptual features are represented not by single neurons but rather by groups of neurons. Face recognition is an example of something that appears to be coded by populations of neurons (Koch, 2004, p. 31). While some groups of cells respond to facial expression (anger, fear, etc.), others respond to the particular angle at which the face is seen.

In addition to the firing rate code assumed in "grandmother cell" and population coding, many researchers postulate an additional temporal coding dimension. To see why, consider the following. A discussion of population coding naturally raises the question of how spatially distributed individual neurons are united to form a population. In line with the firing rate view, one way different cells can be united is by sharing anatomical connections. The assembly could be formed when the firing rates between connected neurons increase. But, appealing to this strategy brings about another problem. How could a population coding for one feature of an object (like facial expression) be distinguished from another population coding for the same feature simultaneously belonging to a different object? Since both populations will increase their firing rates in response to the objects, there will be no way of telling the populations apart (Revonsuo, 2006, p. 272). In response to this problem, many appeal to temporal coding strategies involving the synchronous firing of cells (or "neural synchrony"). ${ }^{36}$ Even though firing 
rate coding is better understood than the more global neural synchrony, many believe that the latter shows more promise in handling the unity of consciousness (as we'll see in Chapter 4).

Let's assume that increased firing rate between interconnected neurons could get around the problem of distinguishing between two populations of neurons coding for the same feature belonging to two different objects. Even ignoring this problem, we'll still need a story as to how multiple objects could be bound together if local approaches are to account for the unity of consciousness.

\section{9 - Could receptive field analyses lead the way to a mechanistic explanation of unity?}

At the level of individual neurons, neural coding activity is usually cashed out in terms of "receptive field" responses. Thus, local approaches typically depend on receptive field analyses. A receptive field is the region of sensory space to which a neuron responds (with spiking activity) (Das, 2002, p. 886). ${ }^{37}$ For instance, a retinal cell's receptive field is the area on the retina to which the cell responds when presented with a particular visual stimulus. ${ }^{38}$ Another example of a receptive field is the area on the skin which, when stimulated by touch, triggers a response from a particular somatosensory neuron. ${ }^{39}$ Because a cell's receptive field properties are usually determined by how much it fires when presented with a particular stimulus somewhere in the sensory space, researchers who employ these analyses are typically committed to a firing rate view of coding (Koch, 2004, p. 57). 
Underlying the concept of the receptive field (RF) is the assumption that perceptual experience can be broken down into components. ${ }^{40}$ These atomic components act as the bricks in the edifice of experience. The coding activities of neurons (the neurons' RF activities) will correlate with these smaller components or atoms of experience. RF studies are appealing because it seems that adding the RF activities of the neurons that respond to an individual feature of a percept to the receptive field activities of the cells which respond to the percept's other features should give us the whole percept's neural correlate. If the RF activity of a neuron somehow codes for a feature or atom experience, the hope is that by determining the RF activities responsible for these atoms of experience, we could eventually account for whole experiences.

What fuels this view? Like the cortex more generally, sensory pathways are thought to be organized hierarchically. Neurons early in a sensory pathway, like those in the retina, respond to simple stimuli (like relative amounts of light detected by different photoreceptor populations) (Koch, 2004, p. 50). Cells which come later in the pathway, such as those in inferior temporal cortex or IT, have larger receptive fields and respond to much more complex stimuli (some cells in IT respond to faces). ${ }^{41}$ This discovery led to a picture of sensory systems as hierarchically organized into processing stages of progressively growing complexity. On this view, the neuronal circuits at each processing stage underpin progressively more complex receptive fields which eventually results in more complex sensory percepts.

This hierarchical picture of sensory systems certainly seems like a step in the direction of providing a mechanistic explanation. The reason it looks like RF activities 
bring us closer to mechanisms of visual consciousness is because a main characteristic of mechanisms is that they too are hierarchically structured. And in a mechanistic explanation, the parts and functioning of a lower layer serve to explain the functioning of the next layer up. This seems to be the sort of explanation that RF analyses could provide.

Let's say we're looking for the mechanism(s) underlying the unified visual experience of a black triangle. We find a cell that vigorously reacts when the triangle is presented in the middle of its RF (but doesn't react to it when presented in other parts of its RF, or to other stimuli). Simply determining a neuron's RF properties is not to provide a mechanism responsible for the coding of the neuron's preferred stimulus. Discovering this mere correlation between the percept and its putative neural correlate (the neuron and its activity) is not explanatory. The most that could be said is that this neuron reacts to its preferred stimulus much like a light goes on when a switch is flicked. The neuron could just be reacting to the complex stimulus without retaining its smaller "parts" in their particular arrangement. We want to know by what mechanism and how the information about the triangle is being computed. We find an example of this type of explanation in the work of Hubel \& Wiesel on the mechanisms of vision.

Visual information gathered at the retina passes through retinal ganglion cells and LGN (lateral geniculate nucleus) cells which project into the primary visual cortex (or V1). In the late 1950s, Hubel \& Wiesel proposed a simple information processing mechanism (involving neuronal circuits) to explain how information in retinal ganglion cells and LGN cells (neurons in the lower layer) could be transformed to account for the 
information processing apparently being computed in a higher layer (by neurons in V1) (Bechtel, 2001, pp. 230-231).

Using single-cell recording techniques, Kuffler had discovered in the 1940s that some retinal ganglion cells and some cells in LGN have receptive fields with "centersurround" organization (Das, 2002, p. 887). "On-center" cells respond more vigorously to stimuli (light or dark spots) in the center of their receptive field than to stimuli in the concentric area surrounding the center of their receptive field. "Off-center" cells respond more vigorously to stimuli in the surround than to stimuli in the center of their receptive field.

Figure 7 - The Receptive Field of an "On-Center" Cell

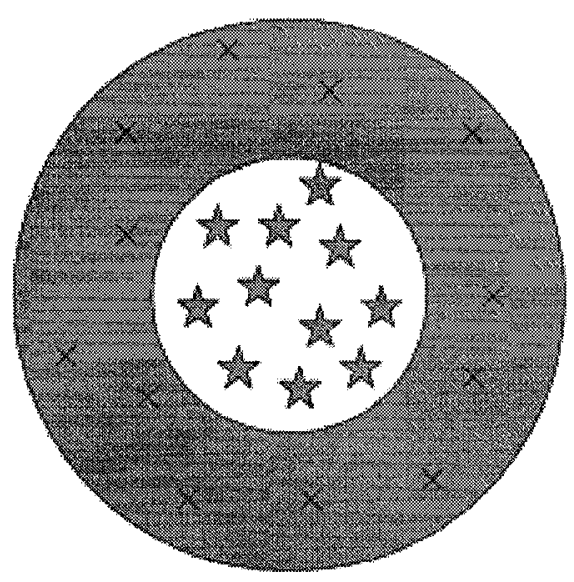

Kuffler had discovered the "center-surround" response properties of retinal ganglion cells and LGN cells by projecting dark spots on light backgrounds and light spots on dark backgrounds. Inspired by the work of Kuffler, Hubel \& Wiesel set out to map the response properties of V1 neurons. They had initially assumed that cells in V1 
would react either to dark spots on light backgrounds or to light spots on dark backgrounds, as the retinal ganglion cells and LGN cells do. However, this hunch proved incorrect - they could not elicit responses from V1 neurons using these stimuli. Then, as Hubel was changing a slide, it accidentally stuck in the projector with the result that a bar rather than a spot of light was projected. The neuron from which they had been recording responded vigorously to this fortuitous stimulus (Bechtel, forthcoming, p. 12). ${ }^{42}$

Using the new bar stimulus, Hubel \& Wiesel discovered what they called "simple cells" and "complex cells." These cells in V1 respond to specifically oriented bars of light or darkness and they are organized in regular patterns in the cortex (Bechtel, forthcoming, p. 13). "Simple cells" respond to bars only in specific areas of their receptive field. Hubel \& Wiesel proposed wiring diagrams explaining how several cells (retinal ganglion/LGN cells) with center-surround sensitivity situated in a straight line could combine their spiking activities to signal the presence of an edge or a bar of light of a specific orientation presented in a specific area of the simple cell's RF. 


\section{Figure 8 - Simple Cell Circuit}

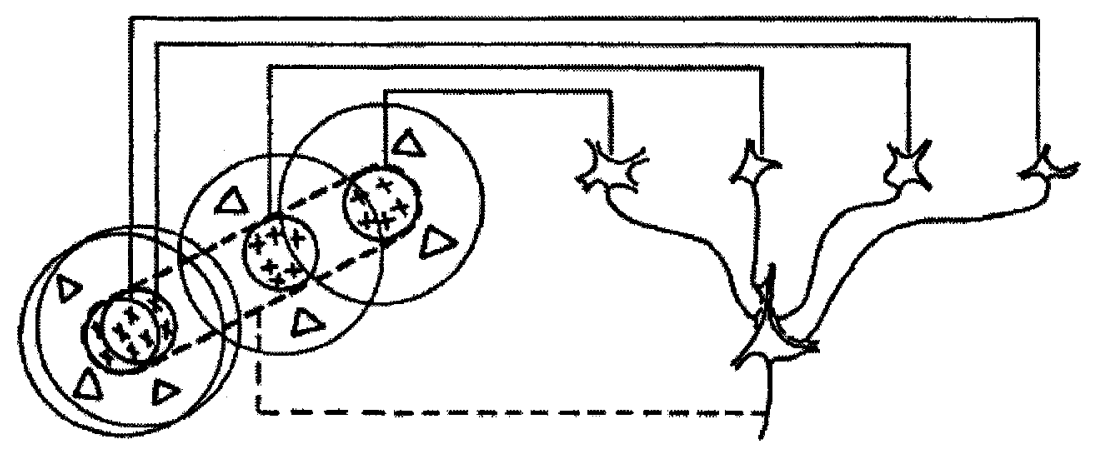

The activities of the "on-center" cells (on the left) are combined to explain the activity of the simple cell (on the right). The simple cell acts as an "and-gate" (with a threshold of three), firing only if it receives spikes from the three cells on the left. Thus, this "and" function would explain the simple cell's activity whenever bars of light in a particular direction are shone on a particular area on the retina (the area where the bar falls on the center of the receptive fields of the three cells on the left).

\section{Figure 9 - Complex Cell Circuit}

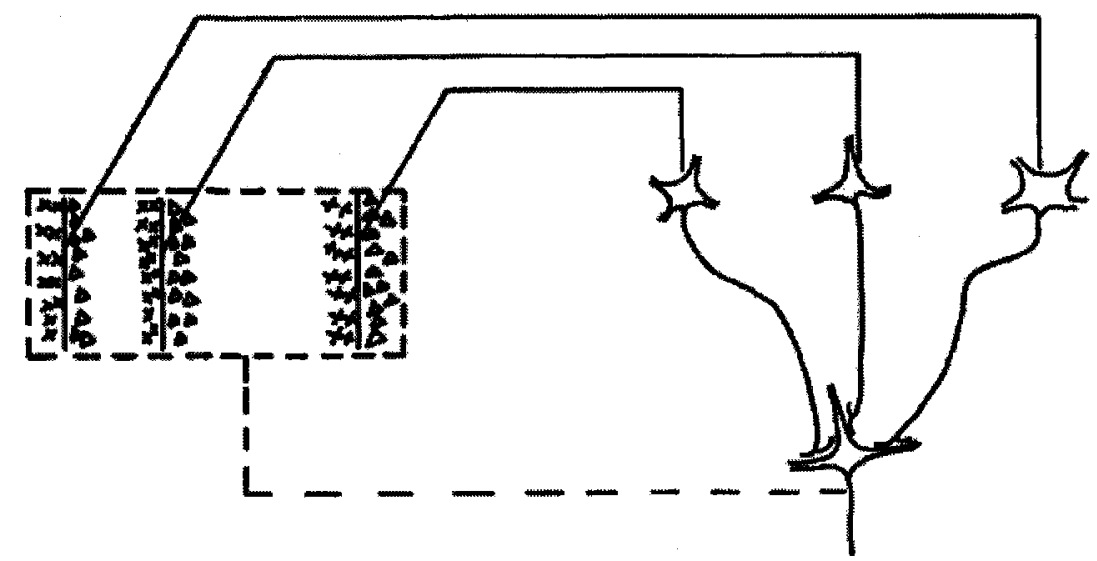

"Complex cells" respond to specifically oriented bars anywhere in their receptive field. The complex cell on the bottom right (Figure 9) functions as an "or-gate" (with a threshold of one) so that the firing of any one of the simple cells on the top right is sufficient to cause it to fire. The receptive fields of the simple cells in this example all have the same vertical orientation (they all react to vertical bars). But, they fall on different parts of the retina. The rectangle represents the area of the retina to which the complex cell reacts - it is its receptive field. 
The important thing to notice in this example is that Hubel \& Wiesel do more than simply map out the receptive fields of neurons. They explain how neuronal activity in a higher layer (V1) could result from the computations of a lower layer (retinal ganglion/LGN cells). So the activity of the V1 neuron in response to the bar stimulus would not just be like a light going off when a switch is flicked. It would be the result of computations at a lower layer of mechanism. ${ }^{43}$

Notice that the structural and functional sides of the mechanisms are specified. Retinal ganglion cells, LGN cells, simple and complex cells (the parts) are structured/organized in way that allows for the realization/computation of a function - the function of combining dots into the more complex edges. (Hubel \& Wiesel's proposal depends on the assumption that retinal ganglion cells and LGN cells somehow code for spots of light because they react to them.)

Hubel \& Wiesel provide a good example of a neuronal mechanism on the local scale. But, the binding of dots into a line or bar involves a very low level of unity. Problems for local approaches quickly arise when we consider conscious experiences involving higher levels of unity. Imagine that a patch of snow completely fills my field of vision. Although the crocus poking up through it clearly appears to me as an object different than the snow, the flower and the snow are distinguishable aspects of the same unified scene. Let's assume that we could distinguish the two separate assemblies that code for the crocus and the snow. Simply knowing that both assemblies increase their firing rates in response to the two objects respectively could tell us that the flower and snow are being experienced. But, this seems to leave out the phenomenological fact that 
they are experienced as part of the same scene: the experience of $x$ and $y$ together is not simply an aggregate of two atomic experiences (Brook \& Raymont, 2006, p. 16).

James offered a thought-experiment which brings out this characteristic of unity between multiple objects (Brook \& Raymont, 2006, p. 16). He asks us to imagine twelve people, each being conscious of a single word from a sentence. No matter how much each person concentrates on their word or how the group arranges itself (in single file, close together, holding hands...), there will be no consciousness of the sentence as a unified whole (James, 1890, Vol.1 p. 160). ${ }^{44}$ Thus, if the unified experience of $x$ and $y$ is not merely the sum of two discrete experiences, the NCC of the experience of $x$ and $y$ cannot merely be the sum of the discrete coding activities separately underlying $x$ and $y-$ we also need an explanation as to how they are united. Therefore, it seems that the NCCs of the unified experience of the crocus on the snow should include more than the discrete coding activities of the flower and those of the snow. Notice that the view of RFs as hierarchically structured into different layers is of no aid here. Even knowing how each layer of coding activities progressively "builds up" to $x$ and to $y$ wouldn't help because $x$ and $y$ are each "built up" separately. Proposing separate mechanisms of $x$ and $y$ will not be enough. We still need an explanation of how the mechanisms unite their information.

\section{$3.10-$ Conclusion:}

In light of the framework set out in Chapter 2, to restrict the search for the mechanisms of unified consciousness to the local levels implied by the rate code and receptive field analyses is to search too low. Explanations at these levels don't seem to 
connect with unified conscious phenomena. Even though local approaches employing the rate code seemed to hold the promise of a mechanistic explanation of very low levels of unity by exploiting the hierarchical structure of RF analyses, it seems that they cannot cope with the binding which is involved in more unified forms of consciousness. One problem they face is how to distinguish between two neuronal populations coding for the same feature in two different objects. Even if we grant that a mechanism of increased firing rate between interconnected neurons could account for the unity of an individual object, local mechanisms appear inadequate to explaining the global unity holding between many objects. As illustrated with James' thought experiment, postulating individual mechanisms for the consciousness of individual objects is not enough for explaining consciousness of multiple objects. Many believe that more global approaches, which invoke temporal synchrony, promise such explanations. In Chapter 4, we turn to synchrony to see if it could complement the rate code and account for the unity of consciousness. Following this examination and critique of synchrony, we conclude our project by proposing an experimental protocol for focusing in on the mechanisms of unified consciousness. 


\section{On the Search for the Neural Correlates of Unified Consciousness}

\section{Chapter 4}

\section{$4.1-\underline{\text { Introduction }}$}

Global approaches to the neural correlates of unified consciousness often appeal to neural synchrony. We begin with a discussion of synchrony in general: what it is, how it is measured, and its proposed role in "selection" (which is necessary to consciousness). Then we consider synchrony as a putative mechanism of unity. In particular, we examine a group of synchrony studies which present empirical evidence tying synchrony at $40 \mathrm{~Hz}$ to the "individual object unity" function. Based on the correlation between $40 \mathrm{~Hz}$ synchrony and the consciousness of unified objects, the authors propose that synchrony at $40 \mathrm{~Hz}$ is responsible for the binding of features into unified objects. However, on our analysis, these studies fail to distinguish the "individual object unity" function from the "multiple object unity" function. We suggest experimental means for dissociating the above two functions. We conclude our project with the suggestion of an experimental protocol which aims to be more specific from both functional and structural perspectives when compared to existing global studies of the neural correlates of unified consciousness.

\section{2 - Synchrony in general}

NCC researchers who appeal to neural synchrony generally do so as a strategy for dealing with the binding problem(s). Recall that the binding of features and objects which results in unified conscious experience at the phenomenal level implies the integration of 
distributed information on the neural level. On the assumption that the brain somehow encodes perceptual information, there must be a neural mechanism responsible for the integration of this spatially distributed information. Milner (1974) and von der Malsburg (1981) proposed synchronization in firing rate (synchrony) as a way that the neurons and their activities could solve the binding problem(s). Because of its putative role in binding, synchrony is hypothesized to be a mechanism underlying unified consciousness.

With neural synchrony, the population of neurons coding for a particular feature would still increase its firing rate in response to the feature. That is, percepts are still assumed to somehow be coded by (increased) firing rate. But, with synchrony, what codes for the features of a percept (rate code) is separated from what unites populations (synchrony). Synchrony in firing discharges is proposed to be what tags the cells belonging to a particular population of neurons.

Synchrony on a millisecond timescale has been detected at both small and large scales of neural organization. Neurons that are close together tend to fire synchronously. But synchrony has also been discovered between neurons that are much farther apart. It has, for instance, been detected between neurons in different cerebral hemispheres (Engel, 2003, p. 138).

Cells firing in a synchronous manner usually do so in a recurrent rhythm. That is, although synchrony can be dissociated from oscillatory rhythms in principle, neural synchrony usually implies oscillatory (or periodic) rhythms (Koch, 2004, p. 45). A neural oscillatory rhythm is a recurrent regular pattern of synchronous activity (Revonsuo, 2006, p. 272). Oscillatory rhythms are described by the frequency of the synchronous spiking. 
Oscillations vary in frequency from one to roughly one hundred $\mathrm{Hz}$ (cycles per second) (Koch, 2004, p. 39). One can think of the frequency of an oscillation as the average speed of the rhythm (calculated as the average number of synchronous spikes occurring in a second).

\section{$4.3-$ How synchrony is measured}

Oscillatory activity is reflected in rhythmic electrical brain potentials that can be measured at the scalp level by EEG (electroencephalography) and MEG (magnetoencephalography). EEG measures the electrical activity generated by neurons. It primarily picks up electrical activity (due to excitation and inhibition) at the neurons' membranes (synapses and dendrites) (Picton \& Mazaheri, 2002, p. 1083). While EEG measures changes in electrical field potentials, MEG measures changes in magnetic fields reflecting the synchronous activity of synapses. The changing currents at the neuronal membranes generate electric fields which can be measured at the scalp with electrodes ("local field potentials"). A local field potential is the electrical potential detected by an electrode placed on the surface of the scalp. The electrode measures the combined synchronous activity of thousands of cells within roughly a millimeter of brain tissue. Local field potentials reflect changes in the average amplitude of a particular frequency band (Revonsuo, 2001, 10).

Averaging techniques can be used to distinguish the brain activity related to a particular stimulus from other EEG activity (Picton \& Mazaheri, 2002, p. 1086). When local field potentials are averaged over hundreds of presentations of a stimulus, we get 
the "evoked potential." This is the average potential evoked by the presentation of a stimulus or by a cognitive event (it is also called an "event-related potential") (Koch, 2004, p. 339). The evoked potential reflects brain activity which was elicited in response to stimulation, as opposed to spontaneous brain activity (more on this distinction in 4.4). When using local field potentials to get "evoked potentials," researchers first choose the frequency band whose presence and amplitude variations they want to detect. But, by using mathematical techniques, the relative amplitudes of many different frequencies can be compared.

Applying mathematical tools to the data collected from electrodes on the scalp allows researchers to represent the amplitudes of a spectrum of frequencies (TallonBaudry \& Bertrand, 2002, p. 256). The "Fourier transform," a mathematical operation, allows for the EEG signal to be transformed into a graph that plots the amplitudes of the various frequencies being recorded (Picton \& Mazaheri, 2002, p. 1083). "Spectral power" graphs provide the comparative amplitudes of oscillations at various frequencies recorded in response to a stimulus. Based on the peaks (which frequencies have the highest amplitudes), the dominant rhythms can be determined.

\section{4 - Spontaneous oscillations versus oscillations elicited by specific stimuli}

When there are no sensory inputs, spontaneously occurring oscillations of varying frequencies can be measured by EEG or MEG. For example, in non-dreaming sleep spontaneous oscillations in the 4-12 $\mathrm{Hz}$ range (spindle rhythm) and the $3-4 \mathrm{~Hz}$ range (delta rhythm) can be detected. The different sleep stages are distinguished on the basis 
of changes in brain rhythm. Both in periods of REM sleep and during wakefulness, the frequency spectrum shifts to the faster gamma range (Wang, 2002, p. 273). (The gamma range includes activity from 20 to $100 \mathrm{~Hz}$. But, because gamma rhythms exhibit a peak around $40 \mathrm{~Hz}$ it is customary to use " $40 \mathrm{~Hz}$ " activity as shorthand for activity between 20 and $100 \mathrm{~Hz}$.) The fact that these spontaneous oscillations can be detected at any recording site on the scalp suggests that they reflect the brain's global state of arousal (TallonBaudry \& Bertrand, 2002, p. 256).

Spontaneous activity at $40 \mathrm{~Hz}$ is sometimes used as an index of a general state of consciousness (as when one is conscious in the sense of being awake and "alert," as opposed to being in a dreamless sleep or coma). To anesthetists, minimal activity at $40 \mathrm{~Hz}$ is an indicator that a patient is unlikely to report having regained consciousness during surgery. ${ }^{45}$ Koch rightly cautions that the link between a general state of consciousness and spiking at $40 \mathrm{~Hz}$ does not tell us how or why this oscillatory activity relates to consciousness. To illustrate this point, he provides the following analogy. If the current from the AC adaptor for your laptop drops below $50-60 \mathrm{~Hz}$, your computer will shut down. Knowing your computer won't function without electrical activity at 50$60 \mathrm{~Hz}$ doesn't tell us how this activity relates to any specific functions it can perform (Koch, 2004, p. 43). If one is interested, not in general states of consciousness, but in more specific conscious percepts, investigating spontaneous oscillations will not do. Oscillations which appear to be stimulus- and task-dependent, are more appropriate. In the mid 1900s, Adrian discovered that gamma oscillations in the rabbit olfactory bulb could be elicited by odour stimuli. He discovered that certain neural 
subpopulations selectively respond to particular odours with transient synchronization (i.e., lasting a few hundred milliseconds) in the gamma band (Wang, 2002, p. 273). In contrast to spontaneous oscillations, oscillations elicited by stimuli are measured at recording sites which are topographically distributed on the scalp in a modality- and taskdependent manner. This implies that synchrony is confined to a subset of neurons rather than to the brain's global state (Tallon-Baudry, 2003a, p. 162). Researchers attempt to correlate the activity of these subsets of neurons with specific cognitive or conscious functions (such as the "selection" of one neuronal population over another or the “individual object unity" function).

\section{5 - Synchrony: A necessary and/or sufficient condition for consciousness?}

In a classic paper, Crick \& Koch (1990) hypothesized that the contents of visual consciousness could be identified with neurons firing in synchrony at $40 \mathrm{~Hz}$. Inspired by studies such as Gray \& Singer's (1989) and Gray et al.'s (1989) (discussed in 4.6), they suggested that this activity was a sufficient condition for consciousness of particular stimuli (individual unified objects). They proposed that some type of attentional mechanism is involved in the transient synchronous firing of distributed neurons. This synchronization at $40 \mathrm{~Hz}$ would bind the neurons responsible for a percept into an assembly, and also activate short-term memory, so that the percept would become conscious (Crick \& Koch, 1990, p. 263). While Crick \& Koch now suggest that synchrony at $40 \mathrm{~Hz}$ may be a necessary condition for the "selection" of one neural assembly over another, they no longer consider synchrony a sufficient condition for 
consciousness (Koch, 2004, p. 46). ${ }^{46}$ Selection is the mechanism by which one assembly rather than another is selected for further cognitive processing (which can lead to consciousness). Assuming that neuronal assemblies code for conscious stimuli, for a stimulus to enter one's globally unified consciousness, the assembly responsible for it must be selected. Because of its role in selection, synchrony in the gamma range would be a necessary condition for consciousness of a percept.

There is much left to be filled out in this proposal. First, it is unclear what is supposed to be doing the selecting. What is the precise role of the attentional mechanism? Is this mechanism different from synchrony? Second, even if it were clear what is making the selection, we also want to know "for whom" it is being made. If we assume that the synchronous firing of a neuronal population is a signal for binding, how could this signal be read-out by other neurons (Roskies, 1999, p. 8)? That is, how and by what could it be decoded $?^{47}$

How should we approach the NCCs of selection? The experimental paradigm of binocular rivalry is well-suited to studying the NCCs of selection. Recall that in this paradigm two different stimuli are presented to the left and right eyes. These stimuli are incongruent such that they cannot be fused into a coherent percept. A normal human subject experiences the two stimuli alternately but never at the same time. While one eye is dominant and the stimulus becomes conscious, the stimulus presented to the other eye is suppressed. That is, the "winning" stimulus is part of one's globally unified consciousness, while the suppressed stimulus is not. What is interesting is that the information provided to the eyes (each stimulus) remains the same while conscious 
experience changes. Thus one can look for changes in neural activity that correlate with the information about a particular stimulus being selected and becoming conscious (becoming part of one's globally unified consciousness).

There is empirical support for the idea that synchrony may be necessary to selection (and thus to consciousness). In a study of binocular rivalry in the awake cat, Fries et al. show that firing rate can be dissociated from synchrony. They found that the synchronous action of $\mathrm{V} 1$ neurons reacting to the winning stimulus increased in synchrony while no increase in firing rate was recorded (Fries et al., 1997, p. 12703). Patterns (vertical gratings) moving in opposite directions were simultaneously presented to each eye. Based on the eye movements of the animals, perceptual dominance was inferred. Eye movements towards the left were assumed to signal consciousness of the left stimulus. ${ }^{48}$ When the left eye was dominant, the corresponding neurons increased in synchrony while the amount of synchrony diminished among neurons driven by the right eye. So neurons reacting to the winning stimulus increased in synchrony while those reacting to the suppressed stimulus decreased in synchrony.

In Chapter 2, we discussed experiments by Sheinberg \& Logothetis (1997) in which macaques were trained to respond behaviourally to stimuli in a binocular rivalry paradigm. The monkeys' behavioural responses show that information about the winning stimulus was selected for further processing because perceptual information about this stimulus was integrated with other cognitive and motor systems. Thus, requiring a behavioural response from the animal would make Fries et al.'s experiment better suited 
to studying the neural correlates of selection. (Sheinberg \& Logothetis measured increases in the firing rate of neurons, not synchronous activity.)

Nevertheless, Fries et al.'s results appear to signal a link between synchrony and selection. But, their experimental paradigm doesn't directly address the issue of the unity of consciousness. The stimuli used were patterns made up of parallel vertical lines. With this experimental design there's no way to tell if a cat's brain activation is a reaction to the discrete (non-unified) features of the patterns or to global characteristics of the patterns as unified objects (such as coherent motion). ${ }^{49}$ The studies that are of most interest to us explicitly examine the possible roles of synchrony in the unity of consciousness.

\section{6 - Synchrony as a putative mechanism of unity}

Oscillations at $40 \mathrm{~Hz}$ have been proposed to be relevant to many different types of cognitive acts. It has been suggested they are important to linguistic processing, associative learning, and memory (Miltner et al., 1999, p. 434). Here, we examine their proposed roles as mechanisms underlying the binding necessary to unified conscious experience.

Many have speculated that synchrony in the gamma range is responsible for binding features into unified objects. ${ }^{50}$ It would realize the binding necessary to our experience of unified objects by integrating the relevant information from the various neural systems involved. Some, like Tallon-Baudry, suggest that synchrony is functionally relevant not only to small scale information integration (and its 
corresponding low levels of phenomenal unity), but to large scale information integration and phenomenal unity in general (Tallon-Baudry, 2003a, p. 157). Indeed, synchrony of various forms and at different frequencies has been invoked as a possible mechanism underlying the information integration responsible for many different levels of conscious unity. ${ }^{51}$ These levels range from the unity of unified individual objects in a specific modality to the unity of our rich multimodal experience of many objects. For example, Engel and Singer believe that synchrony could result in the integration of information from different neural systems as well as across modalities (Engel \& Singer, 2001, p. 24). Varela and Thompson even suggest that synchrony could subserve "the overall integration of all dimensions of a cognitive act, including associative memory, emotional tone, and motor planning" (Varela \& Thompson, 2003, p. 270) (italics in original).

A look at the literature on the NCCs of unified consciousness can make it seem like synchrony is a promising candidate for accounting for various forms of unified consciousness. We hope to show that this enthusiasm is unwarranted, and that synchrony is not a good candidate for being a mechanism of unified consciousness. We now present some empirical evidence from animal and human studies which appear to show a link between synchrony and the information integration necessary to experiencing unified objects. We will restrict ourselves to studies linking synchrony to the function of "individual object unity." Even though we focus on the fairly low level of object unity, the analysis of synchrony's shortcomings also applies to its putative roles in higher levels of unity. 
The first empirical support for the idea that the synchronous activity of neurons correlates with the consciousness of individual objects came from Gray \& Singer's (1989) studies on cats. In response to an individual moving stimulus, neurons in spatially separate columns of the cat visual cortex (Brodmann's areas 17 and 18) displayed synchronized activity in the gamma range (Gray \& Singer, 1989, p. 1698). ${ }^{52}$

Further support came from Gray et al. (1989) whose results are more relevant to the unified characteristics of unified objects. Two electrodes were placed in a cat's visual cortex. When two stimuli (bars) were moved in different directions across the cells' corresponding receptive-fields (one bar to each receptive field) the cells responded with unsynchronized spiking activity in the gamma range. When the two bars were moved in the same direction, there was a slight increase in $40 \mathrm{~Hz}$ synchronization. However, when the two bars were replaced by a single bar which was moved in an area where the cells' receptive fields overlapped, there was a significant jump in $40 \mathrm{~Hz}$ synchronization between the neurons at both recording sites. This suggests that synchrony in the gamma band correlates with global aspects of unified stimuli such as coherent motion (Gray et al., 1989, p. 335). Thus, synchronous firing of the spatially separate cells is hypothesized to somehow be responsible for the integration of the information underlying the featurebinding necessary to the consciousness of the unified percept.

These and similar experiments on animals have motivated experiments on humans using non-invasive techniques such as EEG or MEG. For instance, Gray 's (1989) paradigm was adapted for use with human subjects by Müller and colleagues. A long moving bar elicited larger gamma oscillations than two independently moving stimuli 
(Müller et al., 1996, p. 96). Using humans as subjects is particularly interesting because we can be more assured that the data collected are the results of conscious experience and not of some unconscious reaction to a stimulus. ${ }^{53}$

In order to show that gamma responses systematically correlate with the unity or coherence of a stimulus, responses should be compared in conditions where stimuli share common physical characteristics, yet differ as to whether or not they lead to the perception of unified objects. Should this correlation hold, coherent stimuli which result in unified percepts should provoke stronger gamma responses than incoherent stimuli which do not (Tallon-Baudry, 2003b, p. 356). These four studies report an increase in gamma responses upon presentation of coherent stimuli.

(1) Rodriguez et al. recorded the electrical brain activity of subjects who were presented with "Mooney faces." (“Mooney faces” are figures which look like faces when seen in the upright position but look like incoherent black and white shapes when seen upside down.) Using EEG, they recorded transient gamma responses roughly $230 \mathrm{~ms}$ after presentation of the stimuli. The response was significantly larger in the condition where coherent faces were seen than in the condition where the stimuli were seen as incoherent shapes (Rodriguez et al., 1999, p. 431). (2) Keil et al. used a rotating stimulus which appears as a face when in vertical positions (upright and upside-down). The stimulus appeared incoherent in the other positions. Gamma band activity was largest in response to the positions leading to a unified face percept (Keil et al., 1999, p. 7156). (3) Tallon-Baudry and her group presented three different stimuli to subjects. The two stimuli leading to coherent percepts (a triangle and an illusory triangle) elicited large 
transient synchrony in the gamma range in comparison to a stimulus leading to an incoherent percept (Tallon-Baudry et al., 1996, p. 4246). (4) Revonsuo and colleagues used a paradigm involving illusory stereograms. When sight of the unified percept was reported, a significant increase in transient $40 \mathrm{~Hz}$ synchrony was recorded (Revonsuo, 2006, p. 280).

These results seem to signal a correlation between increased gamma band synchrony and the experience of object unity. These experimental paradigms allowed for a dissociation between consciousness of discrete (non-unified) features and consciousness of individual unified objects. Thus, activity at $40 \mathrm{~Hz}$ seems to correlate with the function of "individual object unity" (the function which binds features into unified objects).

Could $40 \mathrm{~Hz}$ synchrony be the mechanism behind the unity of objects? Three points should throw doubt on this. First, synchronous oscillations at $40 \mathrm{~Hz}$ are proposed to be responsible for the binding of features into objects (among other functions) because they are the most prominent frequency elicited in response to this function. But, many other frequencies are also present at the same time (but at lower amplitudes). It is possible that activity at $40 \mathrm{~Hz}$ may not be the only relevant frequency. For example, maybe delta waves which are oscillating at the same time but at a lower amplitude are also relevant to the function under investigation.

Second, conscious experience can change so rapidly that synchrony appears too slow to keep the pace. Would synchrony be fast enough for you to avoid a stray puck flying towards you? If the puck's colour (or its shape) isn't very quickly bound to its 
movement, you will not be able to move out of the way. The contents of consciousness can change on a millisecond timescale. For example, being shown an image for only about 100 milliseconds is enough for most people to recognize the objects or complex scenes in the image (Revonsuo, 2001, p. 7). This means that objects can be bound in 100 milliseconds or less. Neurons firing in synchrony at $40 \mathrm{~Hz}$ are, on average, emitting one synchronous spike every $25 \mathrm{~ms}$. For synchrony to keep the pace with how fast conscious experience can change, it would have to be able to package its "binding message" into only four synchronous spikes. Four spikes does not seem to be an adequate sample which could allow the frequency to be recognized, especially when we remember that synchronous spikes oscillate - they have periodic rhythms but all spikes are not evenly spaced. This makes it seem even less likely that they could constitute a code.

We soon present an experiment involving masked priming which can serve to isolate different functions of unity. In this experiment a prime was briefly presented for $43 \mathrm{~ms}$ (Sohrabi, 2007, p. 48). During the presentation of this prime, there only would have been time for one synchronous spike at a frequency of $40 \mathrm{~Hz}$. Yet, the priming effects show that the prime's features were bound into a unified object. The "individual object unity" function can happen with a presentation as brief as $43 \mathrm{~ms}$. How could synchrony at $40 \mathrm{~Hz}$ account for this?

Third, another basic problem involving synchrony in general, is how spiking frequency, which is a dynamic process, could encode static information. Lots of conscious information doesn't change that much, like consciousness about certain facts (one's name, what city one lives in, etc.). We raised this problem in the context of coding 
in general when we asked how information in the form of spikes could be stored over time (for use in memory systems). Suffice to note here that synchrony doesn't seem to be the sort of coding strategy which could provide an answer.

\section{7 - What can synchrony tell us about the mechanisms of unified consciousness?}

Discovering brain activity that correlates with or reflects a mechanism does not necessarily bring us closer to that mechanism. Synchrony is not a mechanism in the sense that interests us. Recall that in a mechanistic explanation, a mechanism is decomposed from a functional perspective, and from a structural perspective.

From a functional perspective, mechanistic explanation progresses by decomposing a function into subfunctions or subtasks, and pairing these subtasks to substructures which implement them. As will soon become apparent, even if we knew how to decompose a function into appropriate subtasks, synchrony could not reveal the substructures necessary to explaining the workings of these subfunctions.

In the above animal studies (section 4.6), each electrode recorded the synchronous spiking of a few neurons, and both electrodes were situated within the visual area of the brain. The electrodes used were invasive intracortical electrodes which permit the measurement of the activity of a single neuron or a few neurons (Revonsuo, 2006, p. 322). They revealed local or small scale synchronies. From a structural perspective, these are fine-grained measures with very high spatial resolution. To measure synchrony in humans, non-invasive techniques such as EEG or MEG are used. The electrodes are placed on the scalp and measure the activity of very large groups of neurons. These 
techniques have a very high temporal resolution (in the millisecond range). However, this precision in temporal resolution is offset by poor spatial resolution. From a structural perspective, these are coarser-grained measurements. The oscillations measured by scalp EEG/MEG techniques reveal synchronous oscillatory activity at a large spatial scale which reflects the activity of large neuronal populations. This is because only the synchronous firing of large populations creates a signal strong enough to be picked up by measurements at the scalp level (Tallon-Baudry, 2003b, p. 355). Using EEG/MEG measurements to investigate NCCs is thus to take a global approach.

Oscillations at the scalp level reflect some underlying oscillatory synchrony, but the lack of spatial resolution creates a major problem. The problem is that there's no way to tell, by looking at the data collected by EEG/MEG outside the skull, how and where these patterns were created in the brain (Revonsuo, 2001, p. 14). Based on theoretical models, researchers estimate what the sources of the electrical or magnetic activity were. But the same electric or magnetic field which is recorded could have been created by myriad different combinations of sources inside the brain. ${ }^{54}$ For example, the orientation, phase, and frequency of various sources are all factors which probably combine to determine the amplitude of the signal recorded at a single electrode (Tallon-Baudry, 2003b, p. 358).

Because of the problem of identifying the sources of neural oscillations, synchrony studies and EEG/MEG techniques are not adequate for specifying the structural side of the mechanism of object unity, or of any other function. In other words, the parts of the putative mechanism of synchrony cannot adequately be 
specified/localized. A mechanism's parts must be specified as well as how they work together. If the parts cannot be specified, then we cannot see how they interact to realize a function.

We've noted that mechanistic explanation progresses by decomposing a function into subtasks (or subfunctions). In order to explain the operation of these subtasks, they must be paired to substructures whose workings implement or realize the subtasks (we see how the parts work together in a particular way to perform the subtask). Without the differentiation of relevant neural structures into substructures which implement subtasks, mechanistic explanation cannot progress. Because mechanisms often share systems which do subtasks, the same brain area can be involved in many tasks. Synchrony could not account for this either.

How synchronously firing populations of neurons could be combined into mechanisms that perform tasks (and subtasks) is one problem. How homogenous neural activity (synchronous spiking) could possibly account for the great variety of conscious experiences is another. Notice that this problem of homogeneity is different from the problem of finding the right level of analysis. It affects both global and local approaches. More local approaches also face the problem of how one type of neural activity, action potentials, for instance, could account for the great variety and richness of conscious experiences.

In light of the framework set out in Chapter 2, we can conclude that synchrony studies are carried out at levels of analysis which are not explanatorily useful. They don't 
allow for the decomposition of a mechanism from the structural perspective, and because of this, its functional properties remain hidden.

4.8 - The imaging techniques of fMRI and PET (in combination with TMS) are better for discovering a mechanism's structural and functional properties

The other main brain imaging techniques are fMRI (functional magnetic resonance imaging) and PET (positron-emission tomography). Instead of measuring synchrony, they track changes in the circulatory system. After a short description of these brain imaging techniques, we arrive at what we hope will be the main contribution of our project - the proposal of a methodology for investigating the mechanisms of two different functions of unified consciousness. It involves using fMRI (in combination with transcranial magnetic stimulation or TMS) to measure brain activity during a masked priming paradigm.

On the basis of their different magnetic properties, fMRI detects the relative amounts of oxygenated and deoxygenated blood. This way, fMRI measures increases in blood oxygenation or BOLD (blood-oxygen-level-dependent). PET detects gamma rays generated by a radioactive tracer injected into the bloodstream. Increased presence of radioactive tracer is thought to index increases in blood flow or glucose metabolism. fMRI and PET provide images of changes in the circulatory system. Areas where metabolism increases are assumed to indicate relevant brain activity (Revonsuo, 2001, p. 17). The underlying assumption is that because it correlates to cognitive activity, a type 
of brain activity (glucose and oxygen metabolism) is an indicator of conscious or cognitive activity.

Since they have much better spatial resolution (even though they lack the temporal resolution of EEG/MEG), we recommend that fMRI or PET measures be used when looking for the mechanisms of unity. We think one should favour good spatial resolution over good temporal resolution, because it is much more helpful in localizing the parts involved in the realization of a function (localizing functions from a structural point of view). We recommend that these techniques be used in combination with transcranial magnetic stimulation (TMS). We provide suggestions for using TMS with fMRI which can help identify structural and functional properties of the mechanisms of unified consciousness.

\section{9 - A more fine-grained paradigm (isolating the "individual object unity" function)}

Precisely what cognitive function of unity do the above synchrony studies (p. 97) attempt to isolate and correlate with neural activity? They all claim to investigate the function of uniting features into unified objects (the "individual object unity" function). However, we hope to show that when properly analyzed, it becomes apparent that they fail to distinguish this function from another related, but different, function of unity (the "multiple object unity" function).

All these experiments compare brain activation in two types of conditions: coherent conditions leading to the experience of unified objects and incoherent conditions which do not. In this way they succeed in dissociating consciousness of individual unified 
objects from consciousness of discrete (non-unified) features. Thus, they attempt to get at the "individual object unity" function by dissociating it from the function of consciousness of discrete/non-unified features. However, the problem with this strategy is that it doesn't account for the fact that, in both unified and incoherent conditions, the participant is also in a globally unified conscious state. That is, in both types of conditions, the unified and incoherent stimuli are part of her globally unified conscious state. Thus, each condition really involves (at least) two levels of unity. In the coherent condition we find not only (i) the unity of a single object but also (ii) the higher level unity of this object as it is integrated with the many other objects which comprise one's globally unified state. ${ }^{55}$ Thus, in the above studies, the feature-binding leading to a unified object (the "individual object unity" function), and the integration of this unified object into globally unified consciousness of many objects (the "multiple object unity" function) cannot be distinguished from each other. If we can create conditions that allow for the dissociation of these two levels of unity, we could then look for the neural correlates of the two functions of unity they imply.

We now suggest an experimental paradigm using fMRI which can distinguish between these two functions. We borrow it from Sohrabi (2007) who uses it in a different context. ${ }^{56}$ It is more fine-grained as to what function is being correlated with brain activity because it allows for a more precise distinction between unifying processes. The "individual object unity" function can be dissociated from the "multiple object unity" function. 
Sohrabi administered a line comparison task involving masked and unmasked priming (Sohrabi, 2007, p. 35). The subject's task is to select the longest part of a horizontal line (the target) which is split into two unequal parts. (She does this by pressing one of two buttons ("left" or "right").) Before the target appears, a prime is briefly presented. In this paradigm, the stimuli of interest are the primes (not the targets).

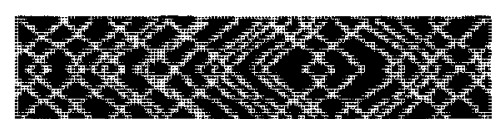

Mask

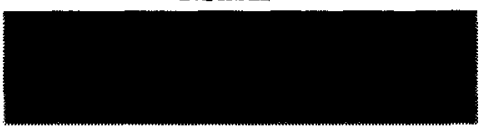

No Mask
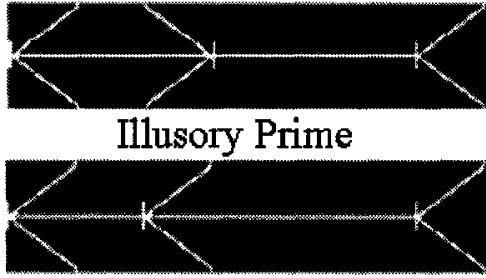

Non-Illusory Prime

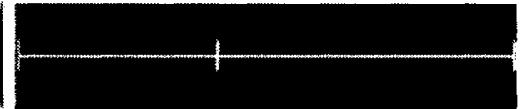

Target (right part is longer)

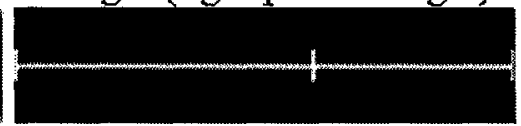

Target (left part is longer)

Figure 10 - Stimuli Used in Illusory Masked Priming Paradigm

In condition 1 (the unmasked condition) the prime becomes part of the subject's globally unified consciousness. In condition 2 (the masked condition) the prime does not become part of the subject's globally unified consciousness. Thus, masking should prevent the operation of the "multiple object unity" function while allowing the operation of the "individual object unity" function.

How do we know if the "individual object unity" function has occurred? If priming effects due to an illusory Gestalt can be measured, then we can infer that the "individual object unity" function has occurred. Illusory Gestalts are used as primes to help ensure that the priming effects reflect the integration of information into a coherent object and are not merely a reaction to isolated features of the primes. The Müller-Lyer 
illusion leads to the experience of a Gestalt. Even though the line is equally divided in two, the arrowheads create the illusion that one side of the prime is longer than the other. ${ }^{57}$ There is a Gestalt relationship between the horizontal line (the "part") and the "whole" which includes the arrowheads. The arrowheads (part of the larger display) affect the way the line (a part) appears. Thus, if one shows priming effects due to the illusion, we can infer that there was consciousness of the object as unified. This is the rationale behind using illusory primes.

What priming effects could we expect to measure? We can measure the "congruency effect." Recall the priming effect discussed in Chapter 2. If two words which share a semantic or associative relation are presented successively, the reaction time for identifying the second word is faster. For example, if "butter" follows "bread," it is usually classified faster than if it follows an unrelated word such as "nurse" (Marcel, 1983, p. 219). In a congruent trial, the prime and the target are similar (like "bread" and "butter" in the classification task). In the case of the present experimental design, a congruent trial is one in which the longest part of the prime appears on the same side (left or right) as the target's longest part. In an incongruent trial, the longest part of the prime appears at the opposite side of the longest part in the target. There is a positive congruency effect when congruent trials are faster than incongruent ones. There is a negative congruency effect when incongruent trials are faster than congruent ones. Sohrabi's results show that the masked illusory prime conditions displayed a positive congruency effect (Sohrabi, 2007, p. 52). 
These priming effects due to the masked Müller-Lyer illusion reflect the unification of the various lines into a unified/coherent object (the figure composed of a line with different arrowheads). The fact that a congruency effect was measured shows that the participant was conscious of the prime as a unified object rather than as a jumble of loose and unrelated features. So priming effects triggered by the masked Müller-Lyer figure reflect the non-reflexively conscious processing of a unified object (corresponding to the "individual object unity" function). Recall the distinction we drew at the beginning of Chapter 2, between consciousness of the world (or "non-reflexive" consciousness) and consciousness of one's own states (or "reflexive" consciousness). To be conscious in the sense of "consciousness of the world" (or "non-reflexive" consciousness) is to be conscious of an item without also being conscious of the state in virtue of which one is conscious of that item. For example, I can be conscious of a table without being conscious of the state in virtue of which I am conscious of the table. When I am conscious of my experience of seeing the table, I am conscious of one of my conscious states ("reflexive" consciousness). One difference between these two general forms of consciousness is the difference between being conscious of $X$ and being conscious of being conscious of $X$. Because there are priming effects but the participant cannot report seeing the prime, we can say that the subject is non-reflexively conscious of the stimulus, but not conscious of being conscious of it (which involves a higher level of unity). The subject is conscious of the masked prime in a way similar to a long-distance driver who is conscious of the road without being conscious of being conscious of it (Armstrong, 1984, p. 58). In contrast, in the unmasked condition, the participant is conscious of being 
conscious of the prime. Because the prime is part of the participant's globally unified conscious state in the unmasked condition, the brain activity in this condition should reflect a higher level of unity (corresponding to the "multiple object unity" function).

We've just explained how the two conditions (masked/unmasked) reflect different functions of unity. But, the "individual object unity" function may also be active during the unmasked condition. (The "individual object unity" function can be seen as a subtask (or subfunction) of the "multiple object unity" function.) Thus, to help ensure that the brain activity in the unmasked condition reflects only the "multiple object unity" function and not the "individual object unity" function as well, we must subtract the brain activation in the masked condition from the activation in the masked condition. Using fMRI, Sohrabi looked for differences in brain activation between the masked and unmasked conditions. We're interested in brain activity which correlates to the primes, not to the target. Unmasked activation minus masked activation should provide the neural correlate (in terms of blood-oxygen-level-dependent or BOLD) of the prime's becoming part of the subject's globally unified conscious state. After subtracting brain activation in the masked illusory prime condition from the activation in the unmasked illusory prime condition, Sohrabi determined that there was significant differential brain activation in the left hemisphere in areas IFG (inferior frontal gyrus) and DLPFC (dorsolateral prefrontal cortex) (Sohrabi, 2007, p. 60). This differential brain activation is the neural correlate of the prime's becoming part of the participant's globally unified consciousness. It is the neural correlate of the "multiple object unity" function. 
4.10 - A more fine-grained paradigm - Second set of experiments (isolating the NCC of the "individual object unity" function)

Sohrabi's experiments suggest that activity in left IFG and DLPFC areas is part of the neural correlate for the function of "multiple object unity." By running a second set of experiments we could search for the neural correlates of the "individual object unity" function. To zero in on this function (which unites features into unified objects), and dissociate it from the function of consciousness of discrete/non-unified features, we should compare brain activation between two conditions. We can use the same paradigm but, this time both conditions are masked so that the primes never become reflexively conscious (the stimuli never become integrated into the participants' globally unified consciousness).

In condition 1 (incoherent condition), the stimulus does not result in an illusory Gestalt. This non-illusory prime should have similar features to the illusory prime from condition 2, but should not result in the (non-reflexively conscious) illusion of a unified object. Because no unified illusory object results, no priming effects should occur. In condition 2 (coherent condition), the stimulus results in an illusory unified object. If the subjects show priming effects, we can infer that the prime resulted in a unified object. So here again priming effects would signal the function of "individual object unity."

Because the stimuli in both conditions are masked and never enter the participants' globally unified consciousness, this set of experiments should help isolate the NCC of the function of "individual object unity" from the possible occurrence of a function of consciousness of discrete/non-unified features. However, unlike the 
experimental designs used in the synchrony studies, our design can isolate the neural correlate of the "individual object unity" function without blurring it with the neural correlate of the "multiple object unity" function. Measuring priming effects due to illusory Gestalts is key to this dissociation.

Thus, the masked illusory prime paradigm allows for the isolation of both the “individual object unity" and "multiple object unity" functions. We now suggest ways in which to use TMS, in conjunction with the masked illusory prime paradigm, to focus in on the mechanisms of our two functions of unified consciousness.

\subsection{1 - $\underline{\text { Transcranial magnetic stimulation (TMS) }}$}

Transcranial magnetic stimulation (TMS) allows for non-invasive stimulation of the cortex through the scalp. This strong magnetic stimulation has the effect of temporarily disrupting the normal functioning of the stimulated area. TMS has much better temporal resolution than fMRI/PET, but its spatial resolution is roughly similar (Walsh \& Cowie, 2000, p. 74). A single TMS pulse appears to affect roughly $1 \mathrm{~cm}^{3}$ of tissue close to the coil which delivers the magnetic field (Robertson et al., 2003, p. 956). Researchers typically start by using fMRI or other imaging techniques to determine the target area to be stimulated. Based on these measurements they establish the position of the coil on the scalp (Sack, 2006, p. 595).

One can take advantage of TMS's good temporal resolution. The normal activity of a brain region can be disrupted for up to $10 \mathrm{~ms}$ by a single pulse which lasts less than 1 ms. When single pulses are used at different points during the execution of a task, the 
exact point, at which normal activity in the target area is crucial for carrying out the task, can be determined (with a temporal resolution between 5 and $10 \mathrm{~ms}$ ) (Sack, 2006, p. 595). So, the precise time at which an area's activity appears necessary to the successful completion of a task can be determined.

\subsection{2 - Why use TMS?}

TMS could be applied to particular brain areas (left IFG and DLPFC) during the execution of the line comparison task. We can use TMS to try and dissociate subsystems appearing to be involved in the functions we isolated. This dissociation can help on at least two fronts: (1) from the structural point of view, by facilitating the more precise identification of the mechanism's parts and (2) from the functional point of view, by allowing for the identification of temporal relationships between subsystems/substructures.

First, TMS could help with the more precise identification of the mechanism's parts. Recall that in the coherent masked prime condition, the stimulus results in an illusory unified object. We can infer that the prime resulted in a unified object if subjects show priming effects. In the masked coherent condition, we could try to knock out the areas identified in Sohrabi's experiment (say the DLPFC or IFG) at different times in relation to the presentation of the prime, and see whether or not priming effects are still measurable.

If effects are still present when TMS is applied to DLPFC, this would suggest that the prime resulted in a unified object, and that the stimulated area is not crucially 
involved in the unification of the illusory object's features. It would suggest that DLPFC is not a part of the minimal NCC for the "individual object unity" function. Conversely, if priming effects were no longer present after TMS, we could infer that some type of activity in DLPFC is probably needed for the feature-binding leading to unified objects. This would suggest that DLPFC is a part of the minimal NCC for the "individual object unity" function.

In this way, even though TMS has spatial resolution similar to fMRI, it could lead to the more precise localization of the neural correlates of functions of unity, by whittling away some of the subsystems that "light up" in imaging studies but which aren't necessary to unity. It could do this primarily because of its active rather than passive nature - TMS interferes with a region's normal activity rather than merely recording it. The combination of its active nature and its high temporal precision allows for this more precise localization. Thus, by revealing dissociations between subsystems, TMS could help localize the parts of mechanisms of unity (from the structural point of view).

Second, turning to the functional point of view, TMS could tell us when the knocked out area's activity appears necessary to performing the function. Because of this, TMS can be used to determine temporal relations existing between the different substructures or subsystems involved in a function. By providing a timeline of this sort, TMS could help us model the functional interactions of the different substructures/subsystems.

For instance, we could use TMS to probe the functional relations between IFG and DLPFC. We could take fMRI measurements while the participant executes the line 
comparison task. Imagine that it turned out that knocking out DLPFC $65 \mathrm{~ms}$ after presentation of the prime (but not before) prevents priming effects. If we also, in a second set of experiments, discovered that knocking out IFG $80 \mathrm{~ms}$ after the presentation of the prime (but not before) prevents priming effects, this would suggest that IFG comes after DLPFC in the processing stream. Further, we could disable DLPFC right after IFG is activated by a prime. If this stimulation of DLPFC (which comes earlier in the stream) prevents the operation of the function, then this would suggest that feedback activity between the two areas is necessary to the conscious function under investigation. (A similar strategy has been used to support the idea that consciousness of motion stimuli depends on feedback relations between areas MT (which comes later in the processing stream) and V1 (which comes earlier) (Lamme, 2003, p. 15).)

The good spatial resolution of imaging techniques can also, somewhat indirectly, help with the decomposition of a mechanism from a functional perspective (by metaanalyses of results from different studies relating to a particular area's functioning). A growing number of studies correlate different conscious functions and cognitive tasks with different brain areas. If, by comparing the data from different studies, we find that a particular brain area is activated by a variety of functions/tasks, one can inquire into what sorts of operations could be common to all these tasks. In this way, imaging studies could contribute to the discovery of new component functions (Bechtel, in press, p. 20). 


\section{$4.13-\underline{\text { Conclusion }}$}

We began our project by defending the viability of functional materialist approaches to consciousness by arguing against defenders of zombie thought experiments. We concluded that these thought experiments are not a threat to functionalism, and that the way is clear for investigating the NCCs in terms of function. We discussed how NCCs are found, and some of the methodological challenges involved in their discovery (such as the use of lesion studies and problems related to the ascription of consciousness). Then we discussed mechanistic explanation and some of the methodological problems it raises, such as how to discover the proper level of organization at which to aim one's analysis. We noted that in mechanistic explanations, mechanisms are decomposed from both structural and functional perspectives. With this dual decomposition as a goal, we set out to evaluate local and global approaches to the NCCs, to see whether they could bring us closer to the mechanisms of unified consciousness. We concluded that local approaches examined very low levels of neural organization whose activity seems unconnected to the unified conscious phenomena we are interested in. It was also apparent that local approaches are inadequate for dealing with some of the challenges raised by the unified character of consciousness such as the "binding problem(s)." We then discussed more global approaches to the NCCs involving "synchrony," and argued that synchrony studies do not bring us closer to the mechanisms of unified consciousness. Synchrony does not allow for the identification of the mechanism's parts - substructures cannot be identified in order to explain the realization of subtasks. We discussed a particular group of studies which proposed $40 \mathrm{~Hz}$ synchrony 
as the putative mechanism of the "individual object unity" function. We concluded that these studies fail to distinguish the "individual object unity" function, from the "multiple object function." Following the description of an experimental paradigm which allows for the dissociation of these two functions, we ended our project with some suggestions for further experimental interventions aimed at zeroing in on the neural correlates of unified consciousness.

As witnessed by the many methodological and conceptual challenges raised by the search for the neural correlates of unified consciousness, uncovering the mechanisms of unified consciousness is far from unproblematic. By approaching the search for the neural correlates of unified consciousness through the framework of mechanistic explanation, our aim was to illustrate what sort of explanation of consciousness we should strive for. By proposing an experimental protocol which explicitly focuses on main desiderata of such an explanation (structural and functional specification of the system's properties), we hope to have made a modest contribution towards reaching that goal. 


\section{Notes}

\section{Chapter 1}

${ }^{1}$ Assuming that no natural phenomenon engenders this unexpected result, for e.g. the presence of a tornado, twister, etc...

${ }^{2}$ The work in this chapter is heavily influenced by our reading of Andrew Brook and Paul Raymont's A Unified Theory of Consciousness (especially their Ch. 3).

${ }^{3}$ Note that thoughts, hopes, and dreams are paradigmatically mental experiences. In saying that the zombie can "have" thoughts and hopes, what is really meant is that it exhibits the behaviour we usually associate with these states.

${ }^{4}$ Braddon-Mitchell attributes this distinction to Chalmers (Braddon-Mitchell, 2003, p. 129).

5 "Aha!" the objector exclaims, "By conceding that it is conceivable that zombies are possible you are, by the same token, proving that zombies are possible." Let us follow our objector's reasoning. Assuming that conceivability implies possibility, saying that (a) "we can conceive of the possibility of $X$ " amounts to saying that (b) "it is possible that $X$ is possible." Thus, it is established that "it is possible that zombies are possible," which is to say that (c) "zombies are possible." However, if one grants the move from (b) to (c), one does so at the expense of rendering incoherent the notion of possibility. Following this reasoning, we can arrive at the conclusion that round squares and married bachelors are possible. A notion of possibility according to which even impossibility is a possibility is patently incoherent. Note that the same faulty reasoning could also be applied to "one can conceive of conceiving $X$," but that the same disastrous consequences regarding the notion of possibility would follow.

${ }^{6}$ We could say that zombies are merely "indirectly conceivable."

${ }^{7}$ See Andrew Brook (1975) where the need for a similar standard is established in the context of thought experiments pertaining to personal identity.

${ }^{8}$ Brook and Raymont address this objection in their forthcoming work. Andrew Brook and Paul Raymont, A Unified Theory of Consciousness.

${ }^{9}$ Chalmers champions a radical dualism according to which matter has dual aspects: both physical properties and phenomenal properties (Chalmers, 2002, p. 265). Note that this sort of dualism could be compatible with materialism in a broad sense.

${ }^{10}$ The ventral stream is associated with visual recognition while the dorsal stream is associated with motor response.

${ }^{11}$ Visual masking paradigms are described in Chapter 2.

\section{Chapter 2}

${ }^{12}$ We adapt this example from Armstrong's famous "long-distance driver" example (Armstrong, 1984, p. 58).

${ }^{13}$ In a chapter entitled "The Primary Visual Cortex as a Prototypical Neocortical Area," Koch summarizes data relating to the structure and function of the primary visual cortex 
and "by proxy, most cortical areas" (Koch, 2004, p. 85). So he clearly believes that other areas will share the same types of structures and mechanisms. (Bartels \& Zeki also think that the mechanisms involved in visual consciousness may also be at work in the brain's functioning more generally (Bartels \& Zeki, 1998, p. 2327).)

${ }^{14}$ Reflecting the "under conditions C" clause which we saw in Chalmers' definition (2.5), Koch adds that it must be specified "over what range of circumstances and data the correlation between neuronal events and conscious percepts holds" (Koch, 2004, p. 17). ${ }^{15}$ In Chapter 4, we suggest more ways in which TMS could be used in an attempt at dissociating different systems appearing to be involved in functions of unified consciousness.

${ }^{16}$ Because its effects are only temporary, transcranial magnetic stimulation could control for the long term reorganization/compensation of the brain following a lesion. It also has the advantage of being more precise in the area it affects than lesions are (Bechtel, in press, p. 8).

${ }^{17}$ In Chapter 4, we suggest a masked priming paradigm for isolating different functions of unity. We'll see that the reason our particular experimental design allows for the dissociation of different functions of unity is because it allows for a dissociation between (a) neural correlates of consciousness of one's own conscious states from (b) neural correlates of world consciousness.

${ }^{18}$ Chalmers believes that NCCs play the functional role of mediating "global availability" (see Baars, 1988; Chalmers, 1998).

${ }_{19}$ Intra-cortical recordings are sometimes performed on human volunteers who are preparing to undergo surgery to treat severe forms of epilepsy. These patients sometimes require intracranial monitoring to locate the exact source of epileptic seizures (Lachaux et al., 1999, p. 199).

${ }^{20}$ For example, the animals were anesthetized in the experiments of (Gray et al., 1989) and (Gray \& Singer, 1989). Both studies are cited in Chapter 4.

${ }^{21}$ For an exception to this in the context of NCC research, see Fell et al. (2004) who approach the NCCs from within the framework of the deductive-nomological model of explanation. This model involves statements in the form of general laws. The main idea behind the deductive-nomological model of explanation is that from a set of initial conditions and these laws, one could deduce future states of the phenomenon under investigation. The hope is that by applying these general laws to a set of initial conditions in the language of neuroscience (describing the NCC and any other of the organism's relevant states), we could predict the organism's conscious states.

${ }^{22}$ Most of Brodmann's divisions have been modified based on physiological criteria that were not available in his time, but some of them are still used today (Koch, 2004, p. 117). ${ }^{23}$ The methodology we propose for getting at the mechanisms of unified consciousness (in Chapter 4), makes use of the technique of fMRI. Although fMRI on its own will not reveal a conscious function's mechanism, we suggest ways that it can be used in combination with transcranial magnetic stimulation (TMS) to help identify a mechanism's component functions (or subtasks) and component parts (or substructures). 


\section{Chapter 3}

${ }^{24}$ For taxonomies see: Brook and Raymont, 2006; Bayne and Chalmers, 2003; Tye, 2003.

${ }^{25}$ For a discussion of accounts of the necessary and sufficient conditions for the unity of consciousness see Brook (2004).

${ }^{26}$ Consciousness is also unified across time (diachronic unity) but we'll restrict ourselves to forms of synchronic unity.

${ }^{27}$ Conversely, changing an individual part of the display can alter the experience of the whole (Tye, 2003, p. 13).

${ }^{28}$ Figure 1 and 2 are reproduced from http://www.pinkgodzillagames.com/pinkytsu/pacman-thumb.jpg and http://brain.mada.org.il/images/illusions/kanitza.gif respectively.

${ }^{29}$ Tables 1-3 adapted from Ken Bigalow's helpful discussion of binary addition at: http://www.play-hookey.com/digital/adder.html. Our neuronal addition circuit is based on a (non-neuronal) digital adder circuit from the same source. We've adapted the circuit to function with "McCulloch-Pitts neurons" by constructing neuronal versions of the logic gates used in the regular electronic circuit.

${ }^{30}$ We borrow our symbolism from Anderson 2002, p. 1043.

${ }^{31}$ The two neurons (with thresholds of one) have been added to ensure that the carry output and sum output are produced simultaneously.

${ }^{32}$ It is believed by some that reverbatory activity between connected neurons and longterm potentiation, which were proposed by Hebb, are possible candidates for being mechanisms of memory (Cooper, 2005, p. 886; Rosenzweig, 2002, p. 1109).

${ }^{33}$ Upon repeated presentation of a stimulus, such as a picture or the sound of a voice, the precise number of times a neuron fires in response can vary slightly. The activity of individual neurons is monitored by electrodes inserted into the cortex.

${ }^{34}$ Working at the level of single neurons is to take a local approach. However, it is possible that the stimulation of a single neuron results in a conscious experience because the neuron triggers a chain reaction with many other neurons or neural systems. The single neuron may only trigger the chain of events leading to the conscious experience in virtue of its particular place in a larger interconnected system.

${ }^{35}$ This doesn't imply that the brain doesn't sometimes code in this way. But it does mean that it cannot be the only type of strategy employed.

${ }^{36}$ Neurons firing synchronously also usually do so rhythmically at various frequencies (see Chapter 4). Thus, one way it is hypothesized that a population could distinguish itself from another is by firing at a different frequency. (Only neurons firing together at the same frequency would belong to the same population.) Another possibitity is that two populations oscillating at the same frequency could be kept separate if oscillating out of phase from each other.

${ }^{37}$ The notion of "receptive fields" was suggested by Sherrington in the 1890s. Hartline did much to develop the concept in 1938 by studying individual crab, frog, and cat optic nerve fibers (Das, 2002, p. 886). 
${ }^{38}$ Certain cells are maximally activated by lines of a certain orientation (e.g., in V1), by movement in a certain direction (MT), or by particular wavelengths (V4) (Koch, 2004, p. 55). We mentioned that different groups of cells are responsible for coding different aspects of seen faces. In an area of the fusiform gyrus, there are cells which respond with varying intensities depending on the angle at which a face is seen. A cell could respond maximally when a face is seen slightly turned to the left and may progressively respond less as the face's orientation moves away from its "preferred" position.

${ }^{39}$ The receptive field of a neuron coding for auditory information includes the pitch to which it most vigorously responds as well as whether it responds to sounds coming from the left or right ear (Koch, 2004, p. 57).

${ }^{40}$ In the literature on the unity of consciousness, lines are divided between those who believe that experience can be partitioned in this way ("experiential parts" view) and those who deny that it can ("no experiential parts" view). Those who hold the "experiential parts" view face the problem, illustrated in James' thought-experiment (p. 84), of how the atomic parts are combined into unified experience (Brook \& Raymont, 2006, p. 16).

${ }^{41}$ The receptive fields of cortical neurons were first described by Hubel \& Wiesel in the late 1950s (Das, 2002, p. 886).

${ }^{42}$ Bechtel remarks that the accidental nature of Hubel \& Wiesel's discovery that neurons in V1 respond to bars of light is suggestive of the difficulty in discovering component functions (decomposing a mechanism's functions into subtasks) (Bechtel, forthcoming, p. 12).

${ }^{43}$ The two simple neuronal circuits/mechanisms above can be seen as two smaller (sub)mechanisms in different layers of a larger mechanism.

${ }^{44}$ By making a few minor changes in what the twelve people are thinking about (individual features of a unified individual object, rather than words from a sentence), James' thought-experiment would work equally well in illustrating the point that unified individual objects of consciousness are more than mere aggregates of discrete features. In the case of object unity there must be a story as to how the features are bound.

\section{Chapter 4}

${ }^{45}$ Based on EEG recordings taken during surgery, and mathematical analysis techniques, researchers are able to assess the depth of a patient's general anesthesia. This "consciousness monitoring" technique has been approved for medical use in the United States (Baars, 2001, p. 26).

${ }^{46}$ The masked priming paradigm we discuss at the end of this chapter (in 4.9) shows that selection does not necessarily lead to a percept's becoming part of one's globally unified consciousness (which is the sort of consciousness Crick and Koch are referring to here). Masked priming effects are proof against selection (and whatever its mechanism is) being a sufficient condition for an individual object's becoming part of one's globally unified consciousness. 
${ }^{47}$ This problem is a symptom of the bigger problem of the homogenous nature of synchronous activity (which we address later in this chapter).

${ }^{48}$ In humans there is a near perfect correlation, in binocular rivalry conditions, between direction of eye movement and consciousness of the moving patterns (Engel, 2003, p. 145). It seems reasonable to assume that the cat's eye movements signal some level of perceptual processing.

${ }^{49}$ Coherent motion is a principle of organization that the visual system seems to follow when uniting an object's features. When elements in a visual scene move in a coherent way, they tend to be experienced as perceptual units (Revonsuo, 2006, p. 207).

${ }^{50}$ Some examples: Revonsuo, 2001, p. 14; Tallon-Baudry, 2003b, p. 355; Engel, 2003, p. 134; Noë \& Thompson, 2004, p. 15.

${ }^{51}$ For instance, von Stein \& Sarnthein (2000) used EEG to record synchrony during tasks thought to involve progressively higher degrees of unity. They found that the frequency $(\mathrm{Hz})$ of synchrony seems to decrease as the level of unity (and the underlying scale of information integration) involved in a task increased (von Stein \& Sarnthein 2000, p. 392).

${ }^{32}$ The cells of the sensory cortex are organized into columns perpendicular to the cortical sheet.

${ }^{53}$ The animals in (Gray et al., 1989) and (Gray \& Singer, 1989) were anesthetized which increases the probability that their behaviour was not accompanied by phenomenal consciousness.

${ }^{54}$ Koch suggests that using EEG and related methods to track the NCCs is analogous to trying to infer the structure and depth of an ocean by measuring the waves traveling at its surface (Koch, 2004, p. 41).

${ }^{55}$ In the incoherent condition we find (i) the very low level of unity (or lack thereof) of discrete/non-unified features and (ii) the unity of these features as they are experienced as part of one's globally unified consciousness of many objects.

${ }^{56}$ Sohrabi is primarily concerned with the relations between the factors of masked/unmasked priming, and illusory/non-illusory priming in relation to conflict resolution. What's new and particularly interesting about Sohrabi's research is his use of illusory primes. By using illusory figures, he cuts down on other cognitive processing (past paradigms used number comparison tasks which involved a higher cognitive load), to focus on the activity of the visual system. Although he does not specifically examine various levels of unified consciousness, in virtue of his use of illusory primes, Sohrabi's results are relevant to the search for the mechanisms of unified consciousness.

${ }^{57}$ The fact that the illusion persists even when subjects know it is illusory suggests that the results reflect a bottom-up unifying mechanism. This is significant because, when investigating consciousness, it can be hard to disentangle the possible involvement of top-down mechanisms (such as attention) from bottom-up ones (Frith et al., 1999, p. 112). 


\section{References}

Anderson, J. A. (2002). McCulloch-Pitts neurons. In Macmillan Encyclopedia of Cognitive Science (Vol. 2, pp. 1043-1047). Macmillan Publishers Ltd.

Armstrong, D. M. (1984). The nature of mind. Brighton: Harvester Press.

Baars, B. J. (1988). A cognitive theory of consciousness. Cambridge University Press.

Baars, B. J. (2001). How could brain imaging not tell us about consciousness? Journal of Consciousness Studies, 8(3), 24-29.

Bartels A. \& Zeki, S. (1998). The theory of multistage integration in the visual brain. Proceedings Royal Society London, 265, 2327-2332.

Bayne, T. \& Chalmers, D. J. (2003). What is the unity of consciousness? In A. Cleeremans (Ed.), The unity of consciousness: Binding, integration and dissociation (pp. 23-58). Oxford: Oxford University Press.

Bechtel, W. (2001). Decomposing and localizing vision: An exemplar for cognitive neuroscience. In W. Bechtel, P. Mandik, J. Mundale \& R. S. Stufflebeam (Eds.), Philosophy and the neurosciences: A reader (pp. 225-249). Oxford: Basil Blackwell.

Bechtel, W. (2005a). Mental mechanisms: What are the operations? Proceedings of the 27th Annual Meeting of the Cognitive Science Society.

Bechtel, W. (2005b). The challenge of characterizing operations in the mechanisms underlying behavior. Journal of the Experimental Analysis of Behavior, 84, 313325. 
Bechtel, W. (in press). The epistemology of evidence in cognitive neuroscience. In R. Skipper Jr., C. Allen, R.A. Ankeny, C.F. Craver, L. Darden, G. Mikkelson \& R. Richardson (Eds.), Philosophy and the life sciences: A reader. Cambridge, MA: MIT Press.

Braddon-Mitchell, D. (2003). Qualia and analytical conditionals. The Journal of Philosophy, Vol. C, 3, March, 111-135.

Brook, A. (1975). Imagination, possibility, and personal identity. American Philosophical Quarterly, 12 (3), 185-198. Reprinted in J. M. A. Skorupski (Ed.), International research library of philosophy. Aldershot, England: Dartmouth Publishing, 1992.

Brook, A. (1994). Kant and the mind. New York. Cambridge University Press.

Brook, A. (2004). Kant, cognitive science, and contemporary NeoKantianism. Journal of Consciousness Studies, 11 (10-11), 1-25.

Brook, A. (Forthcoming). The possibility of a cognitive architecture.

Brook, A. \& Raymont, P. The unity of consciousness. In Edward N. Zalta (Ed.), The Stanford Encyclopedia of Philosophy (Fall 2006 Edition). Retrieved June 24, 2007 from http://plato.stanford.edu/archives/fall2006/entries/consciousnessunity/.

Brook, A. \& Raymont, P. (Forthcoming). A unified theory of consciousness. Cambridge, MA: MIT Press.

Chalmers, D. J. (1996). The conscious mind. Oxford: Oxford University Press. 
Chalmers, D. J. (1998). On the search for the neural correlate of consciousness. In S. Hameroff, A. Kaszniak \& A. Scott (Eds.). Towards a science of consciousness II (pp. 219-230). Cambridge, Mass.: MIT Press.

Chalmers, D. J. (1999). Materialism and the metaphysics of modality. Philosophy and Phenomenological Research, 59, 473-493.

Chalmers, D. J. (2000). What is a neural correlate of consciousness? In Metzinger, T. (Ed.), Neural correlates of consciousness: Empirical and conceptual questions (pp. 17-40). Cambridge, MA: MIT Press.

Chalmers, D. J. (2002). Consciousness and its place in nature. In Chalmers, D. J. (Ed.), Philosophy of mind: Classical and contemporary readings (pp. 247-272). Oxford: Oxford University Press.

Churchland, P. (1997). Matter and consciousness. Cambridge, MA: MIT Press.

Cottrell, A. (1999). Sniffing the camembert: On the conceivability of zombies, Journal of Consciousness Studies, 6 (1), 4-12.

Cooper, S. J. (2005). Donald O. Hebb's synapse and learning rule: A history and commentary, Neuroscience and Biobehavioral Reviews, 28, 851-874.

Crick, F. \& Koch, C. (1990). Towards a neurobiological theory of consciousness. Seminars in Neuroscience, 2, 263-275.

Crick, F. \& Koch, C. (1995). Are we aware of neural activity in primary visual cortex? Nature, 375, 121-123.

Crick, F. \& Koch, C. (1998). Consciousness in neuroscience. Cerebral Cortex, 8, 97-107. 
Crick, F. \& Koch, C. (2003). A framework for consciousness. Nature Neuroscience, $6,119-126$.

Das, A. (2002). Receptive fields. In Macmillan Encyclopedia of Cognitive Science (Vol. 3, pp. 886-897). Macmillan Publishers Ltd.

Davidson, D. (1970). Mental events. In Essays on actions and events. Oxford: Oxford University Press, 2001, 207-227.

Dennett, D. (1995). The unimagined preposterousness of zombies. Journal of Consciousness Studies, 2 (4), 322-326.

Dennett, D. (1999). The zombic hunch: Extinction of an intuition? Final Draft for the Royal Institute of Philosophy Millennial Lecture, November 28. From http://ase.tufts.edu/cogstud/papers/zombic.htm.

Engel, A. K. (2003). Temporal binding and neural correlates of consciousness. In A. Cleeremans (Ed.), The unity of consciousness: Binding, integration, and dissociation (pp. 132-152). Oxford: Oxford University Press.

Engel, A. K. \& Singer, W. (2001). Temporal binding and the neural correlates of sensory awareness. Trends in Cognitive Sciences, 5, 16-25.

Fell, J., Elger, C. E. \& Kurthen, M. (2004). Do neural correlates of consciousness cause conscious states? Medical Hypotheses, 63, 367-369.

Földiák, P. (2002). Representation in neurons. In Macmillan Encyclopedia of Cognitive Science (Vol. 1, pp. 339-347). Macmillan Publishers Ltd. 
Fries, P., Roelfsema, P. R., Engel, A. K., König, P. \& Singer, W. (1997). Synchronization of oscillatory responses in visual cortex correlates with perception in interocular rivalry. Proceedings of the National Academy of Sciences USA, 94, 12699-12704.

Frith, C., Perry, R. \& Lumer, E. (1999). The neural correlates of conscious experience: An experimental framework. Trends in Cognitive Sciences, 3, 105-111.

Goodale, M. G. (2004). Perceiving the world and grasping it: Dissociations between conscious and unconscious visual processing. In The Cognitive Neurosciences III, Cambridge, MA: MIT Press.

Gray, C. M., König, P., Engel, A. K. \& Singer, W. (1989). Oscillatory responses in cat visual cortex exhibit inter-columnar synchronization which reflects global stimulus properties. Nature, 338, 334-337.

Gray, C. M. \& Singer, W. (1989). Stimulus-specific neuronal oscillations in orientation columns of cat visual cortex. Proceedings of the National Academy of Sciences USA, 86, 1698-1702.

Hameroff, S. R. \& Penrose, R. (1996). Orchestrated reduction of quantum coherence in brain microtubules: A model for consciousness. Mathematics and Computers in Simulation, 40 (3), 453-480.

Heywood, C. A. \& Milner, D. (2002). Neural correlates of visual consciousness. In Macmillan Encyclopedia of Cognitive Science (Vol. 2, pp. 240-245). Macmillan Publishers Ltd. 
Hohwy, J. (2007). The search for neural correlates of consciousness. Philosophy Compass, 2 (3), 461-474.

Hubel, D. H. \& Wiesel, T. N. (1962). Receptive fields, binocular interaction and function architecture in the cat's visual cortex. Journal of Physiology, 160, 106-154.

Hume, D. (1978). A Treatise of Human Nature. L. A. Selby-Bigge (Ed.), Oxford: Oxford University Press.

Humphreys, G. (2003). Conscious visual representations built from multiple binding processes: Evidence from neuropsychology. In A. Cleeremans (Ed.), The unity of consciousness: Binding, integration, and dissociation (pp. 114-131). Oxford: Oxford University Press.

James, W. (1890). Principles of psychology (Vol.1). London: Macmillan.

Kaczmarek, L. (2002). Neuron doctrine. In Macmillan Encyclopedia of Cognitive Science (Vol. 3, pp. 325-329). Macmillan Publishers Ltd.

Keil, A., Müller, M. M., Ray, W. J., Gruber, T. \& Elbert, T. (1999). Human gamma band activity and perception of a Gestalt. Journal of Neuroscience, 19, 7152-7161.

Koch, C. (2004). The quest for consciousness: A neurobiological approach. Englewood, CO: Roberts \& Co. Publishers.

Kriegel, U. (2006). The same-order monitoring theory of consciousness. In U. Kriegel \& K. Williford (Eds.), Self-representational approaches to consciousness (pp. 143170). Cambridge, MA: MIT Press. 
Lamme, V. A. F. (2003). Why visual attention and awareness are different. Trends in cognitive science, 7 (1), 12-18.

Levine, J. (1983). Materialism and qualia: The explanatory gap. Reprinted in D. J. Chalmers (Ed.), Philosophy of mind: Classical and contemporary readings (pp. 354-359). Oxford: Oxford University Press, 2002.

Levine, D. S., (2000). Introduction to neural and cognitive modeling. New Jersey: Lawrence Erlbaum Associates.

Llinás, R. (2003). Consciousness and the thalamocortical loop. International Congress Series, 1250, 409-416.

Lumer, E. D., Friston, K. J. \& Rees, G. (1998). Neural correlates of perceptual rivalry in the human brain. Science, 280 (5381), 1930-1934.

Marcel, A. J., (1983). Conscious and unconscious perception: Experiments on visual masking and word recognition. Cognitive Psychology, 15, 197-300.

Miller, S. M. (2007). On the correlation/constitution distinction problem (and other hard problems) in the scientific study of consciousness. Acta Neuropsychiatrica, 19 (3), 159-176.

Milner, P. (1974). A model for visual shape recognition. Psychological Review, 81, 521535.

Miltner, W. H. R., Braun, C., Arnold, M., Witte, H. \& Taub, E. (1999). Coherence of gamma-band EEG activity as a basis for associative learning. Nature, 397, 434436. 
Morris, J., Ohman, A. \& Dolan, R. J. (1999). A subcortical pathway to the right amygdala mediating 'unseen' fear. Proceedings of the National Academy of the Sciences USA, 96, 1680-1685.

Müller, M. M., Bosch, J., Elbert, T., Kreiter, A., Sosa, M. V., Sosa, B. \& Rockstroh, B. (1996). Visually induced gamma-based responses in human electroencephalographic activity - a link to animal studies. Experimental Brain Research, 112, 96-102.

Noë, A. \& Thompson, E. (2004). Are there neural correlates of consciousness? Journal of Consciousness Studies, 11, (1), 3-28.

Penfield, W. \& Perot, P. (1963). The brain's record of auditory and visual experience: A final summary and discussion. Brain, 86 (4), 595-696.

Picton T. W. \& Mazaheri, A. (2002). Electroencephalography (EEG) (Vol. 1, pp. 10831087). In Macmillan Encyclopedia of Cognitive Science. Macmillan Publishers Ltd.

Raymore, P. (2002). A materialist response to David Chalmers' The conscious mind. Retrieved December 20, 2006 from http://www.stanford.edu/group/dualist/vol4/pdfs/raymore.pdf.

Revonsuo, A. (2001). Can functional brain imaging discover consciousness in the brain? Journal of Consciousness Studies, 8 (3), 3-23.

Revonsuo, A. (2006). Inner presence: Consciousness as a biological phenomenon. Cambridge, MA: MIT Press. 
Revonsuo, A., Wilenius-Emet, M., Kuusela, J. \& Lehto, M. (1997). The neural generation of a unified illusion in human vision. NeuroReport, 3867-3870.

Robertson, E. M., Théoret, H. \& Pascual-Leone, A. (2003). Studies in cognition: The problems solved and created by transcranial magnetic stimulation. Journal of Cognitive Neuroscience, 15, 948-960.

Rodriguez, E., George, N., Lachaux, J. P., Martinerie, J., Renault, B. \& Valera, F. (1999). Perception's shadow: Long-distance synchronization of human brain activity. Nature, 397, 430-433.

Rosenzweig, M. R. (2002). Memory, neural basis of: Cellular and molecular mechanisms. In Macmillan Encyclopedia of Cognitive Science (Vol. 2, pp. 11051109). Macmillan Publishers Ltd.

Roskies, A. (1999). The binding problem. Neuron, 24, 7-9.

Sabri, M., Kareken, D. A., Dzemidzic, M., Lowe, M. \& Melara, R. D. (2004). Neural correlates of auditory sensory memory and automatic change detection. NeuroImage, 21, 69-74.

Sack, A. T. (2006). Transcranial magnetic stimulation, causal structure-function mapping and networks of functional relevance. Current Opinion in Neurobiology, 16 (5), 593-599.

Sewards, V. \& Sewards, M. A. (2000). The awareness of thirst: Proposed neural correlates. Consciousness and Cognition, 9, 463-487. 
Sheinberg, D. L. \& Logothetis, N. K. (1997). The role of temporal cortical areas in perceptual organization. Proceedings of the National Academy of Sciences USA, 94 (7), 3408-3417.

Sohrabi, A. (2007). Positive and negative congruency effects in masked and unmasked priming: Match of representation strength, attention, and consciousness. Doctoral Dissertation, Carleton University.

Tallon-Baudry, C. (2003a). Oscillatory synchrony as a signature for the unity of visual experience in humans. In Cleeremans A. (Ed.), The unity of consciousness:

Binding, integration, and dissociation (pp. 153-167). Oxford: Oxford University Press.

Tallon-Baudry, C. (2003b). Oscillatory synchrony and human visual cognition. Journal of Physiology, 97, 355-363.

Tallon-Baudry, C. \& Bertrand, O. (2002). Gamma oscillations in humans. In Macmillan Encyclopedia of Cognitive Science (Vol. 2, pp. 255-260). Macmillan Publishers Ltd.

Tallon-Baudry, C., Bertrand, O., Delpuech, C. \& Pernier, J. (1996). Stimulus specificity of phased-locked and non-phase-locked $40 \mathrm{~Hz}$ visual responses in human. The Journal of Neuroscience, 16 (13), 4240-4249.

Tong, F., Nakayama, K., Vaughan, J. T. \& Kanwisher, N. (1998). Binocular rivalry and visual awareness in human extrastriate cortex. Neuron, 21, 753-759.

Tononi, G. \& Edelman, G. (1998). Consciousness and complexity. Science, 282, 18461851. 
Tye, M. (2003). Consciousness and persons: Unity and identity. Cambridge, MA: MIT Press.

Varela, F. \& Thompson, T. (2003). Neural synchrony and the unity of mind: A neurophenomenological perspective. In A. Cleeremans (Ed.), The unity of consciousness: Binding, integration, and dissociation (pp. 266-287). Oxford: Oxford University Press.

Verhagen, J. V. (2006). The neurocognitive bases of human multimodal food perception: Consciousness. Brain Research Reviews, 53, 271-286.

von der Malsburg, C. (1981). The correlation theory of brain function. MPI Biophysical Chemistry, Internal Report, 81-82. Reprinted in Domany, E., van Hemmen, J. L. \& K. Shulten (Eds.), Models of Neural Networks II, Berlin, Springer, 1994. von Stein, A. \& Sarnthein, J. (2000). Different frequencies for different scales of cortical integration: From local gamma to long range alpha-theta synchronization. International Journal of Psychophysiology, 38, 301-313.

Walsh, V. \& Cowey, A. (2000). Transcranial magnetic stimulation and cognitive neuroscience. Nature, 1, 73-79.

Wang, X. J. (2002). Neural oscillations. In Macmillan Encyclopedia of Cognitive Science (Vol. 3, pp. 272-280). Macmillan Publishers Ltd. 


\section{List of Sources of Tables and Figures}

Tables 1 to 3. Adapted from http://www.play-hookey.com/digital/adder.html. Accessed February 13, 2008.

Tables 4 to 7 . By author.

Figure 1. Retrieved March 25, 2008, from http://www.pinkgodzillagames.com/pinkytsu/pacman-thumb.jpg.

Figure 2. Retrieved March 25, 2008, from

http://brain.mada.org.il/images/illusions/kanitza.gif.

Figure 3. Retrieved March 25, 2008, from http://www-

psych.stanford.edu/ lera/psych115s/notes/lecture3/images/neuron.gif.

Figures 4 to 6 . By author.

Figure 7. Adapted from Hubel \& Wiesel, 1962, p. 142.

Figure 8. Reproduced from Hubel \& Wiesel, 1962, p. 142.

Figure 9. Reproduced from Hubel \& Wiesel, 1962, p. 143.

Figure 10. Reproduced from Sohrabi, 2007, p. 46. 


\section{Glossary of science-related terms}

$40 \mathrm{~Hz}$ synchrony: Synchrony in the gamma frequency range $(20-100 \mathrm{~Hz})$, with a peak around $40 \mathrm{~Hz}$. (Also called gamma band synchrony.)

Action potential: A pulse-like change in electrical potential across a neuron's membrane. Also referred to as firing or spiking activity.

Assembly (neuronal): An assembly is a functionally unified group of neurons. (Also called neuronal population.)

Binocular rivalry paradigm: Experimental paradigm in which two different stimuli are presented to the left and right eyes. These stimuli are incongruent such that they cannot be fused into a coherent percept. A normal human subject experiences the two stimuli alternately, but never at the same time. While one stimulus is experienced (the winning stimulus), the other is suppressed (the suppressed stimulus).

BOLD: Blood-oxygen-level-dependent.

DLPFC: Dorsolateral prefrontal cortex.

EEG: See Electroencephalography.

Electroencephalography (EEG): Electroencephalography measures the electrical activity generated by neurons. It primarily picks up electrical activity (due to excitation and inhibition) at the neurons' membranes (synapses and dendrites).

Firing rate coding: Neural coding theory according to which the average number of spikes (or action potentials), recorded over repeated stimulations, is the relevant neural activity.

fMRI: See Functional magnetic resonance imaging.

Functional magnetic resonance imaging (fMRI): Brain imaging technique which measures increases in blood oxygenation. On the basis of their different magnetic properties, fMRI detects the relative amounts of oxygenated and deoxygenated blood.

Gamma band synchrony: Synchrony in the gamma frequency range $(20-100 \mathrm{~Hz})$ with a peak around $40 \mathrm{~Hz}$. (Also called $40 \mathrm{~Hz}$ synchrony.) 
IFG: Inferior frontal gyrus.

LGN: Lateral geniculate nucleus.

Magnetoencephalography (MEG): Magnetoencephalography measures changes in magnetic fields reflecting the synchronous activity of neurons (electric activity at the neurons' synapses).

Masked prime/Masked priming paradigm: In masked priming paradigms, the presentation of a stimulus (the "prime") is quickly followed by the brief presentation of a "mask" (usually a random pattern or some other stimulus different from the prime). If a mask is presented a few $10-100 \mathrm{~ms}$ after the presentation of a stimulus, the participant cannot report consciousness of the stimulus.

MEG: See Magnetoencephalography.

NCC: Neural correlate of consciousness.

"Neuron doctrine": The view that neurons are the fundamental components of the nervous system and that the brain's functioning can be understood by their actions and interactions.

"Off-center" cells: Cells which have receptive fields with "center-surround" organization. Off-center cells respond more vigorously to stimuli in the concentric area surrounding the center of their receptive field than to stimuli in the center of their receptive field.

"On-center" cells: Cells which have receptive fields with "center-surround" organization. On-center cells respond more vigorously to stimuli in the center of their receptive field than to stimuli in the concentric area surrounding the center of their receptive field.

Oscillations (neural): Neural oscillations are recurrent regular patterns of synchronous neuronal activity. Oscillatory rhythms are described by the frequency of the synchronous spiking. Oscillations vary in frequency from 1 to roughly $100 \mathrm{~Hz}$ (cycles per second). They are measured at the scalp with MEG or EEG.

PET: See Positron-emission tomography.

PFC: Prefrontal cortex.

Population (neuronal): A population is a functionally unified group of neurons. (Also called neuronal assembly.) 
Positron-emission tomography (PET): Brain imaging technique which measures changes in the circulatory system. PET detects gamma rays generated by a radioactive tracer injected into the bloodstream. Increased presence of the radioactive tracer is thought to index increases in blood flow or glucose metabolism.

Preferred stimulus: The type of stimulus to which a neuron reacts the most vigorously (by firing action potentials).

Priming effects: Cognitive effects measurable on a task, subsequent to the presentation of a stimulus (the "prime").

Receptive field (RF): A receptive field is the region of sensory space to which a neuron responds (with spiking activity). For instance, a retinal cell's receptive field is the area on the retina to which the cell responds when presented with a particular visual stimulus.

REM: Rapid eye movement.

RF: See Receptive field.

Spike: See Action potential.

Stereogram: Optical illusion of depth in using a two-dimensional image. Before binocular fusion (before the subject focuses their eyes correctly), the stimulus looks like an incoherent assortment of random dots. After binocular fusion, a coherent threedimensional object is seen where no patterns or perceptual groupings appeared before.

Synchrony (neural): The synchronous firing of large groups of neurons. This temporal coding strategy is hypothesized to unite distributed neurons into functional groups.

TMS: See Transcranial magnetic stimulation

Transcranial magnetic stimulation (TMS): Transcranial magnetic stimulation allows for non-invasive stimulation of the cortex through the scalp. This strong magnetic stimulation has the effect of temporarily disrupting the normal functioning of the stimulated area. 